Pure and Applied Mathematics Quarterly

Volume 1, Number 3

(Special Issue: In Memory of

Armand Borel, Part 2 of 3 )

$591-663,2005$

\title{
Compact Clifford-Klein Forms of Symmetric Spaces - Revisited
}

\author{
Toshiyuki Kobayashi and Taro Yoshino
}

\section{In memory of Armand Borel}

\begin{abstract}
This article discusses the existence problem of a compact quotient of a symmetric space by a properly discontinuous group with emphasis on the non-Riemannian case. Discontinuous groups are not always abundant in a homogeneous space $G / H$ if $H$ is non-compact. The first half of the article elucidates general machinery to study discontinuous groups for $G / H$, followed by the most update and complete list of symmetric spaces with/without compact quotients. In the second half, as applications of general theory, we prove: (i) there exists a 15 dimensional compact pseudoRiemannian manifold of signature $(7,8)$ with constant curvature, (ii) there exists a compact quotient of the complex sphere of dimension 1, 3 and 7, and (iii) there exists a compact quotient of the tangential space form of signature $(p, q)$ if and only if $p$ is smaller than the Hurwitz-Radon number of $q$.
\end{abstract}

\section{Contents}

1 Introduction

2 Examples

Received May 12, 2005.

This work is partly supported by Grant-in-Aid for Exploratory Research 16654014, JSPS.

2000 Mathematics Subject Classification. Primary 22F30; Secondary 22E40, 53C30, 53C35, $57 \mathrm{~S} 30$

Key words: discontinuous group, Clifford-Klein form, symmetric space, space form, pseudoRiemannian manifold, discrete subgroup, uniform lattice, indefinite Clifford algebra. 
$2.1 \quad$ Notation and Definition 595

$2.2 \quad$ Symmetric spaces with compact Clifford-Klein forms $\quad 595$

$2.3 \quad$ Para-Hermitian symmetric spaces 596

$2.4 \quad$ Complex symmetric spaces $G_{\mathbb{C}} / K_{\mathbb{C}}$

$2.5 \quad$ Space form problem 599

2.6 Tangent space version of space form problem 600

3 Methods 601

$3.1 \quad$ Generalized concept of properly discontinuous actions 601

$3.2 \quad$ Criterion of properness for reductive groups 605

3.3 Construction of compact Clifford-Klein forms 608

$3.4 \quad$ Obstruction I - Calabi-Markus phenomenon 611

3.5 Obstruction II - maximality of non-compactness 613

3.6 Obstruction III - compatibility of three compact spaces 616

4 Space form problem $\quad 618$

$4.1 \quad$ Space form conjecture $\quad 618$

$4.2 \quad$ Compact Clifford-Klein forms 620

4.3 Clifford algebra associated to an indefinite quadratic form 622

$\begin{array}{lll}4.4 & \text { Clifford algebra } & 624\end{array}$

$\begin{array}{ll}4.5 & \text { Definition and Lemmas }\end{array}$

4.6 Proof of Proposition 4.4.1 630

$\begin{array}{lll}4.7 & \text { Isomorphism } \lambda & 633\end{array}$

$4.8 \quad$ Proof of Proposition 4.4.4 638

$4.9 \quad$ Proof of Theorem 4.2.1 643

$4.10 \quad$ Compact Clifford-Klein form of $S O(8, \mathbb{C}) / S O(7,1)$

$5 \quad$ Tangential space form problem $\quad 645$

$5.1 \quad$ Tangential symmetric space 646

$5.2 \quad$ Discontinuous groups for tangential homogeneous spaces 647

$\begin{array}{lll}5.3 & \text { Reduction to connected subgroups }\end{array}$ 


\section{INTRODUCTION}

A Clifford-Klein form of a symmetric space $M=G / H$ is the double coset manifold $\Gamma \backslash G / H$, where $\Gamma \subset G$ is a discontinuous group for $M$. Geometrically, we are dealing with a complete, locally symmetric space. The aim of this article is to discuss recent progress on the following problem after Borel [Bo63] for the Riemannian case and Kobayashi [Ko88] for the non-Riemannian case.

Problem A. Does a symmetric space $M$ admit compact Clifford-Klein forms?

In his celebrated paper "Compact Clifford-Klein forms of symmetric spaces", Borel proved Problem A affirmatively, but under the tacit assumption that $M$ is Riemannian.

Theorem 1.1.1 ([Bo63]). Compact Clifford-Klein forms always exist if $M$ is Riemannian.

Opposite extremal cases may happen for general symmetric spaces $M$.

Theorem 1.1.2 (see Theorem 2.3.1). A compact Clifford-Klein form never exists if $M$ is para-Hermitian.

Among symmetric spaces, irreducible ones were classified infinitesimally by Berger [Br57]. Even for irreducible symmetric spaces, Problem A has not been completely solved.

In this paper, we shall discuss general machinery to study Problem A, and then apply it to specific symmetric spaces. Then we shall provide a table of (irreducible) symmetric spaces that are proved to admit/not to admit compact Clifford-Klein forms. It includes:

Theorem 1.1.3 (see Section 2.4). The 7 dimensional complex sphere admits a compact Clifford-Klein form. 
Theorem 1.1.4 ([CaM62],[Ku81],[Kl96]; see Fact 2.5.2). There exists an $n$ dimensional compact Lorentz manifold of constant curvature $\kappa$ if and only if $\kappa=0$, or $\kappa<0$ and $n$ is odd.

Theorem 1.1.5 (see Section 4). There exists a 15 dimensional compact complete pseudo-Riemannian manifold of signature $(7,8)$ with constant positive sectional curvature.

These latter two theorems are regarded as a partial answer to the space form problem, which asks whether or not there exists a compact pseudo-Riemannian manifold of general signature $(p, q)$ with constant sectional curvature (see Conjecture 2.5.1). Furthermore, its 'tangential' version for the Cartan motion group is formulated and completely solved. We recall from [Hu23] or [Rd22] that the Hurwitz-Radon number $\rho(q)$ is defined to be $8 \alpha+2^{\beta}$ if we write $q=u \cdot 2^{4 \alpha+\beta}$ (u is odd, $0 \leq \beta \leq 3)$.

Theorem 1.1.6 (see Section 5). The tangential symmetric space of $O(p+1, q)$ $/ O(p, q)$ admits a compact Clifford-Klein form if and only if $p<\rho(q)$.

This article focuses on Problem A for symmetric spaces. For non-symmetric spaces such as $S L(n, \mathbb{R}) / S L(m, \mathbb{R})(n>m)$, in addition to the methods that we shall explain in Section 3, various other approaches have been also made including ergodic theory, symplectic geometry and unitary representations ([Ko92a, 96b], [BnL92], [Co94], [Z94], [LMZ95], [LZ95], [Ma97], [O98], [Sh00], [OW02]), though some of these approaches are not applicable to symmetric spaces. See also recent survey papers [Ma00], [Ko01, 02], [IW04] and references therein.

This article has a survey nature largely in the first half, and gives new results on Problem A in the latter half. It is organized as follows: Section 2 exhibits the most update knowledge on the existence problem of compact Clifford-Klein forms of symmetric spaces. Various examples given in Section 2 are obtained by a general theory to Problem A based on group theoretic study of discontinuous groups and geometric consideration of Clifford-Klein forms. An introduction to the general theory will be the main topic of Section 3. In Section 4, we apply the general theory to the space form problem, and in particular, give a proof of Theorem 4.2.1 (this includes Theorems 1.1.3 and 1.1.5) using Clifford algebras associated to indefinite quadratic forms. Section 5 studies Problem A for the tangential symmetric spaces, and in particular, gives a proof of Theorem 1.1.6.

Professor Armand Borel invited the first author to the Institute for Advanced Study at Princeton with warmest encouragement on the initial stage of this work, right after his visit to Kyoto in 1990, where the first author gave a presentation at the RIMS workshop organized by Professor Satake. It is an honor to dedicate this paper to the memory of Professor Borel. 


\section{Examples}

2.1. Notation and Definition. First, suppose $H$ is a closed subgroup of a Lie group $G$. Assume that $\Gamma$ is a discrete subgroup of $G$ acting properly discontinuously and freely on the homogeneous space $G / H$. Then, the double coset space $\Gamma \backslash G / H$ is Hausdorff in the quotient topology and the quotient map

$$
\pi: G / H \rightarrow \Gamma \backslash G / H
$$

becomes a covering map, so that $\Gamma \backslash G / H$ carries naturally a manifold structure.

Definition 2.1.1. The resulting manifold $\Gamma \backslash G / H$ is said to be a Clifford-Klein form of $G / H$, and the discrete subgroup $\Gamma$ is a discontinuous group for $G / H$. If the double coset space $\Gamma \backslash G / H$ is furthermore compact, we say $\Gamma$ is a cocompact discontinuous group for $G / H$, and we say $G / H$ has a tessellation. A cocompact discontinuous group is also referred to as a uniform lattice for $G / H$ or a crystallographic group for $G / H$.

Second, suppose $\sigma$ is an automorphism of a Lie group $G$ such that $\sigma^{2}=$ id. Then, the set of fixed points $G^{\sigma}:=\{g \in G: \sigma(g)=g\}$ is a closed subgroup of $G$. We write $\left(G^{\sigma}\right)_{0}$ for the identity component of $G^{\sigma}$. The homogeneous space $G / H$ is called a symmetric space if $\left(G^{\sigma}\right)_{0} \subset H \subset G^{\sigma}$. A symmetric space is said to be semisimple [reductive,...] if $G$ is a semisimple [reductive,...] Lie group.

Any Clifford-Klein form of a symmetric space (therefore, a symmetric space itself) becomes a complete locally symmetric space through its canonical affine connection. Conversely, any complete locally symmetric space is represented as a Clifford-Klein form $\Gamma \backslash G / H$ of some symmetric space $G / H$ ([KN69]).

2.2. Symmetric spaces with compact Clifford-Klein forms. Any Riemannian symmetric space admits compact Clifford-Klein forms, as we have seen in Theorem 1.1.1. This result was proved by finding a cocompact discrete subgroup $\Gamma$ of a linear semisimple Lie group $G^{\prime}$. In turn, the group manifold $G / H:=G^{\prime} \times G^{\prime} / \operatorname{diag} G^{\prime}$ also admits a cocompact discontinuous group $\Gamma \times\{e\}$. Since $G^{\prime} \times G^{\prime} / \operatorname{diag} G^{\prime}$ is a symmetric space by the involution $\sigma(x, y):=(y, x)$, this gives another example of symmetric spaces that have compact Clifford-Klein forms.

Apart from these Riemannian symmetric spaces and group manifold cases, there are some more semisimple symmetric spaces that have compact CliffordKlein forms.

Theorem 2.2.1 (see Corollary 3.3.7). The following semisimple symmetric spaces $G / H$ admit compact Clifford-Klein forms $(n=1,2, \ldots)$. 


\begin{tabular}{c|c} 
& $G / H$ \\
\hline 1 & $S U(2,2 n) / S p(1, n)$ \\
2 & $S U(2,2 n) / U(1,2 n)$ \\
3 & $S O(2,2 n) / U(1, n)$ \\
4 & $S O(2,2 n) / S O(1,2 n)$ \\
5 & $S O(4,4 n) / S O(3,4 n)$ \\
6 & $S O(4,4) / S O(4,1) \times S O(3)$ \\
7 & $S O(4,3) / S O(4,1) \times S O(2)$ \\
8 & $S O(8,8) / S O(7,8)$ \\
9 & $S O(8, \mathbb{C}) / S O(7, \mathbb{C})$ \\
10 & $S O(8, \mathbb{C}) / S O(7,1)$ \\
11 & $S O^{*}(8) / U(3,1)$ \\
12 & $S O^{*}(8) / S O^{*}(6) \times S O^{*}(2)$
\end{tabular}

Table 2.2

Clearly, the same conclusion still holds if we replace $G$ and $H$ by locally isomorphic groups up to finite coverings and finitely many connected components. For example, the cases 11,12 and $S O(2,6) / U(1,3)$ are locally isomorphic to each other.

2.3. Para-Hermitian symmetric spaces. We say a manifold $M^{2 n}$ of even dimension has a paracomplex structure if the tangent bundle $T M$ splits into a Whitney direct sum $T^{+} M \oplus T^{-} M$ with equi-dimensional fibers and if $T^{ \pm} M$ are completely integrable. A pseudo-Riemannian metric $g$ over a paracomplex manifold $M$ is said to be a para-Hermitian metric if $T_{x}^{ \pm} M \subset T_{x} M$ are maximal totally isotropic subspaces with respect to $g$ for every point $x$ in $M$. A semisimple symmetric space equipped with $G$-invariant para-Hermitian structure is said to be a para-Hermitian symmetric space. Kaneyuki and Kozai [KK85] characterized para-Hermitian symmetric spaces by the property that the center $C$ of $H$ is noncompact, and gave a classification of irreducible ones based on the latter property.

Concerning Problem A, a para-Hermitian symmetric space is an opposite extreme to a Riemannian symmetric space:

Theorem 2.3.1. None of para-Hermitian symmetric spaces admits a compact Clifford-Klein form.

Proof. If $G / H$ is a para-Hermitian symmetric space, then $H$ has the non-compact center $C$ and its Lie algebra $\mathfrak{h}$ coincides with the centralizer of $C$ in $\mathfrak{g}$. Since any 
maximal split abelian subspace $\mathfrak{a}$ of $\mathfrak{g}$ contains $C, \mathfrak{a}$ is contained in the centralizer of $C$ in $\mathfrak{g}$, namely $\mathfrak{h}$. Therefore $\mathbb{R}$ - $\operatorname{rank} G=\mathbb{R}$ - rank $H$. Now, apply Theorem 3.4.1 (the Calabi-Markus phenomenon).

Example 2.3.2. Here are some examples of para-Hermitian symmetric spaces $G / H$. In particular, there are no compact Clifford-Klein forms of these $G / H$ :

$$
\begin{array}{c|c} 
& G / H \\
\hline 1 & S L(p+q, \mathbb{R}) / S(G L(p, \mathbb{R}) \times G L(q, \mathbb{R})) \\
2 & S p(n, \mathbb{R}) / G L(n, \mathbb{R}) \\
3 & S O^{*}(4 n) / U^{*}(2 n) \\
4 & S U^{*}(2(p+q)) / S\left(U^{*}(2 p) \times U^{*}(2 q)\right) \\
5 & S p(n, n) / U^{*}(2 n) \\
6 & S U(n, n) / S L(n, \mathbb{C}) \times \mathbb{R} \\
7 & S O(n, n) / G L(n, \mathbb{R}) \\
8 & S O(p, q) / S O(p-1, q-1) \times S O(1,1)
\end{array}
$$

Table 2.3

Remark 2.3.3. Any para-Hermitian symmetric space is realized as a hyperbolic orbit of the adjoint action on the Lie algebra $\mathfrak{g}$ (see [Ko98b]). Hence, Theorem 2.3.1 can be regarded as a special case of the following theorem (see [Ko92b, Theorem 1.3]): a semisimple orbit with a compact Clifford-Klein form must be an elliptic orbit and have a G-invariant complex structure. Two different proofs are known for this theorem; the original proof was based on the criterion of properness (see Theorem 3.5.1), while Benoist and Labourie [BnL92] gave an alternative proof based on symplectic geometry.

2.4. Complex symmetric spaces $G_{\mathbb{C}} / K_{\mathbb{C}}$. A symmetric space $G / H$ is a complex symmetric space if $G$ is a complex Lie group and $\sigma$ is an involutive holomorphic automorphism of $G$ such that $\left(G^{\sigma}\right)_{0} \subset H \subset G^{\sigma}$.

One of typical examples of complex symmetric spaces are $S O(n+1, \mathbb{C}) / S O(n, \mathbb{C})$, which is biholomorphic to the $n$-dimensional complex sphere

$$
S_{\mathbb{C}}^{n}:=\left\{\left(z_{1}, \ldots, z_{n+1}\right) \in \mathbb{C}^{n+1}: z_{1}^{2}+\cdots+z_{n+1}^{2}=1\right\} .
$$

Theorem 2.4.1. The $n$-dimensional complex sphere $S O(n+1, \mathbb{C}) / S O(n, \mathbb{C})$ admits a compact Clifford-Klein form if $n=1,3$ or 7 .

Proof. Clear in the case $n=1$ because $S O(2, \mathbb{C}) / S O(1, \mathbb{C}) \simeq \mathbb{C}^{*} /\{1\}$. For $n=$ $3, S O(4, \mathbb{C}) / S O(3, \mathbb{C})$ is locally isomorphic to the group manifold $S O(3, \mathbb{C}) \times$ $S O(3, \mathbb{C}) / S O(3, \mathbb{C})$ and therefore admits a compact Clifford-Klein form as we 
saw in Section 2.2. The proof for the case $n=7$ will be postponed until Section 4 (see Theorem 4.2.1).

We pose:

Conjecture 2.4.2. The $n$-dimensional complex sphere $S O(n+1, \mathbb{C}) / S O(n, \mathbb{C})$ admits a compact Clifford-Klein form if and only if $n=1,3$ or 7 .

Remark 2.4.3. The condition $n=1,3$ or 7 appears as a necessary and sufficient condition that there exist $n$ vector fields on the sphere $S^{n}$ which are linearly independent at every point, as was proved by Bott-Milnor, Kervaire, and AtiyahHirzebruch ([BtM58], [Ke58], [AtH61]). This is not just a coincidence. In fact, we can prove that the tangential symmetric space (see Definition 5.1.2) of $S_{\mathbb{C}}^{n}$ admits a compact Clifford-Klein form if and only if $n=1,3$ or 7 based on this result. In Section 5, we shall see the relation between Problem A and vector fields on the sphere in another setting arisen from space form problem.

The complex sphere $S O(n+1, \mathbb{C}) / S O(n, \mathbb{C})$ is an irreducible complex symmetric space if $n=2$ or $n \geq 4$. The local classification of irreducible complex symmetric spaces is equivalent to the classification of real simple Lie algebras, and there are 10 classical series (see Table 2.4) and 22 exceptional ones (É. Cartan 1914). Locally, irreducible complex symmetric spaces are obtained as 'complexification' of irreducible Riemannian symmetric spaces. Hereafter, we shall write $G_{\mathbb{C}} / K_{\mathbb{C}}$ for the complex semisimple symmetric space if it is a complexification of the Riemannian symmetric space $G / K$. For the reader's convenience, we present a list of classical cases:

\begin{tabular}{r|c|c} 
& $G / K$ & $G_{\mathbb{C}} / K_{\mathbb{C}}$ \\
\hline 1 & $S L(n, \mathbb{R}) / S O(n)$ & $S L(n, \mathbb{C}) / S O(n, \mathbb{C})$ \\
2 & $S U^{*}(2 n) / S p(n)$ & $S L(2 n, \mathbb{C}) / S p(n, \mathbb{C})$ \\
3 & $S U(p, q) / S(U(p) \times U(q))$ & $S L(p+q, \mathbb{C}) / S(G L(p, \mathbb{C}) \times G L(q, \mathbb{C}))$ \\
4 & $S O_{0}(p, q) / S O(p) \times S O(q)$ & $S O(p+q, \mathbb{C}) / S O(p, \mathbb{C}) \times S O(q, \mathbb{C})$ \\
5 & $S O^{*}(2 n) / U(n)$ & $S O(2 n, \mathbb{C}) / G L(n, \mathbb{C})$ \\
6 & $S p(n, \mathbb{R}) / U(n)$ & $S p(n, \mathbb{C}) / G L(n, \mathbb{C})$ \\
7 & $S p(p, q) / S p(p) \times S p(q)$ & $S p(p+q, \mathbb{C}) / S p(p, \mathbb{C}) \times S p(q, \mathbb{C})$ \\
8 & $S L(n, \mathbb{C}) / S U(n)$ & $S L(n, \mathbb{C}) \times S L(n, \mathbb{C}) / S L(n, \mathbb{C})$ \\
9 & $S O(n, \mathbb{C}) / S O(n)$ & $S O(n, \mathbb{C}) \times S O(n, \mathbb{C}) / S O(n, \mathbb{C})$ \\
10 & $S p(n, \mathbb{C}) / S p(n)$ & $S p(n, \mathbb{C}) \times S p(n, \mathbb{C}) / S p(n, \mathbb{C})$
\end{tabular}

Table 2.4 
We recall from Borel's result (Theorem 1.1.1) that any Riemannian symmetric space $G / K$ admits a compact Clifford-Klein form (see the left-hand side). In contrast, the following seems plausible:

Conjecture 2.4.4. An irreducible complex symmetric space $G_{\mathbb{C}} / K_{\mathbb{C}}$ admits a compact Clifford-Klein form if and only if $G_{\mathbb{C}} / K_{\mathbb{C}}$ is locally isomorphic to either $S O(8, \mathbb{C}) / S O(7, \mathbb{C})$ or a group manifold.

It should be noted:

Proposition 2.4.5. Conjecture 2.4.2 is equivalent to Conjecture 2.4.4.

Proof. What remains to prove is that an irreducible complex symmetric space $G_{\mathbb{C}} / K_{\mathbb{C}}$ does not admit a compact Clifford-Klein form if $G_{\mathbb{C}} / K_{\mathbb{C}}$ is not locally isomorphic to $S O(n+1, \mathbb{C}) / S O(n, \mathbb{C})$ or a group manifold. By using Theorem 3.5.1, we proved this statement holds in [Ko92a, Example 1.9] except for the three cases $G_{\mathbb{C}} / K_{\mathbb{C}} \approx S O(2 m+2, \mathbb{C}) / S O(2 m+1, \mathbb{C}), S L(2 m, \mathbb{C}) / S p(m, \mathbb{C})$ and $E_{6, \mathbb{C}} / F_{4, \mathbb{C}}$ (see also Corollary 3.5.9). Furthermore, Benoist [Bn96] proved that $S O(4 m+2, \mathbb{C}) / S O(4 m+1, \mathbb{C})$ and the latter two cases do not have compact Clifford-Klein forms (see Corollary 3.4.7). Hence, Proposition is proved.

As in the above proof, Conjecture 2.4.2 is now reduced to showing the nonexistence of a compact Clifford-Klein form for $S O(4 m, \mathbb{C}) / S O(4 m-1, \mathbb{C}) \quad(m \geq$ $3)$. We remark that $S O(4, \mathbb{C}) / S O(3, \mathbb{C})$ and $S O(8, \mathbb{C}) / S O(7, \mathbb{C})$ admit compact Clifford-Klein forms by Theorem 2.4.1.

2.5. Space form problem. A space form is a pseudo-Riemannian manifold $M$ with constant sectional curvature. This is another example of locally symmetric space. It is an unsolved problem for which signature of indefinite metric there exists a compact space form. We give the following:

Conjecture 2.5.1 ([Ko01, Conjecture 2.6]). There exists a compact complete pseudo-Riemannian manifold of signature $(p, q)$ with constant sectional curvature $\kappa$ if and only if $(p, q, \kappa)$ satisfies one of the following conditions.

1) $\kappa>0$ and the pair $(p, q)$ is in the list below:

\begin{tabular}{c||c|c|c|c|c}
$p$ & $\mathbb{N}$ & 0 & 1 & 3 & 7 \\
\hline$q$ & 0 & $\mathbb{N}$ & $2 \mathbb{N}$ & $4 \mathbb{N}$ & 8
\end{tabular}

2) $\kappa=0$ and the pair $(p, q)$ is arbitrary.

3) $\kappa<0$ and the pair $(p, q)$ is in the list below:

\begin{tabular}{c||c|c|c|c|c}
$p$ & 0 & $\mathbb{N}$ & $2 \mathbb{N}$ & $4 \mathbb{N}$ & 8 \\
\hline$q$ & $\mathbb{N}$ & 0 & 1 & 3 & 7
\end{tabular}


We say $M$ is complete if the geodesic is defined for all the time interval $(-\infty, \infty)$. A complete space form of signature $(p, q)$ with constant sectional curvature $\kappa$ is a Clifford-Klein form of the following symmetric spaces:

1) $(\kappa>0) \quad O(p+1, q) / O(p, q) \quad(p \geq 2) \quad$ or $\quad \widetilde{O(2, q)} / \widetilde{O(1, q)} \quad(p=1)$.

2) $(\kappa=0) \quad O(p, q) \ltimes \mathbb{R}^{p+q} / O(p, q)$.

3) $\quad(\kappa<0) \quad O(p, q+1) / O(p, q) \quad(q \geq 2) \quad$ or $\quad \widetilde{O(p, 2)} / \widetilde{O(p, 1)} \quad(q=1)$.

Thus, Conjecture 2.5.1 gives a conjectural answer to Problem A for the above symmetric spaces.

The 'if' part of Conjecture 2.5.1 has been proved by Borel, Kulkarni and Kobayashi (Theorem 1.1.1 and Corollary 3.3.7). Obvious cases for this are the case $q=0$ in (1) because the sphere $S^{p}$ itself is compact, and the case (2) because $\mathbb{R}^{p+q} / \mathbb{Z}^{p+q}$ is a compact flat space form. The 'only if' part remains unsolved though in spite of some recent progress that provides evidence supporting the conjecture (Corollaries 3.4.2, 3.4.7 and 3.6.5).

Conjecture 2.5.1 is known to be true, particularly for the Riemannian and Lorentzian cases (i.e. $p \leq 1$ or $q \leq 1$ ). To be more precise:

Fact 2.5.2 ([CaM62],[Ku81],[K196]). Concerning n-dimensional Lorentz space forms of sectional curvature $\kappa$ :

1) $(\kappa>0)$ Compact forms never exist.

2) $(\kappa=0)$ Compact forms always exist.

3) $(\kappa<0)$ Compact forms exist if and only if $n$ is odd.

This fact can be proved as special cases of Corollary 3.4.2 $(\kappa>0)$, and Corollaries 3.3.7 and 3.6.5 $(\kappa<0)$. The case $\kappa=0$ is clear, as we have already seen that $\mathbb{Z}^{k+1} \backslash \mathbb{R}^{k+1}$ is a compact flat space form. Note that compactness implies completeness for the Lorentz space form by a theorem of Klingler ([K196]).

Section 4 will summarize the current status of Conjecture 2.5.1 for general signature $(p, q)$, and will explain its 'if' part including a complete proof of Theorem 1.1.5, namely, the existence of a compact space form of signature $(p, q)=$ $(7,8)$.

2.6. Tangent space version of space form problem. Associated to a reductive Lie group $G$ with a Cartan decomposition $\mathfrak{g}=\mathfrak{k}+\mathfrak{p}$, one defines the Cartan motion group $G_{\theta}:=K \ltimes \mathfrak{p}$ as a semidirect group. Likewise, one can associate a (non-reductive) symmetric space $G_{\theta} / H_{\theta}$ to a reductive symmetric space $G / H$, which we say the tangential symmetric space.

Problem A for the tangential symmetric space $G_{\theta} / H_{\theta}$ turns out to be much simpler than that for the reductive symmetric space $G / H$. In fact, we can solve 
a 'tangential' version of 'space form conjecture' (Conjecture 2.5.1). To formulate the result, we recall the Hurwitz-Radon number $\rho(n)$ (see [Hu23], [Rd22]) defined as follows: A positive integer $n$ is uniquely written as $n=u \cdot 2^{4 \alpha+\beta}$, where $u, \alpha$ and $\beta$ are non-negative integers, $u$ is odd and $\beta \leq 3$. Then, the Hurwitz-Radon number is given by

$$
\rho(n):=8 \alpha+2^{\beta} .
$$

It is useful to set $\rho(0):=\infty$.

Let $G=O(p+1, q)$ and $H=O(p, q)$. Then Theorem 1.1.6 asserts that the tangential symmetric space $G_{\theta} / H_{\theta}$ has a compact Clifford-Klein form if and only if $p<\rho(q)$. Then, we obtain the list of the pair $(p, q)$ such that $G_{\theta} / H_{\theta}$ has a compact Clifford-Klein form.

\begin{tabular}{c||c|c|c|c|c|c|c|c|c|c}
$p$ & $\mathbb{N}$ & 0 & 1 & 2 & 3 & 4 & 5 & 6 & 7 & $\cdots$ \\
\hline$q$ & 0 & $\mathbb{N}$ & $2 \mathbb{N}$ & $4 \mathbb{N}$ & $4 \mathbb{N}$ & $8 \mathbb{N}$ & $8 \mathbb{N}$ & $8 \mathbb{N}$ & $8 \mathbb{N}$ & $\cdots$
\end{tabular}

We may observe that the above list is different from, but somewhat close to the original space form conjecture (see Conjecture 2.5.1 for $\kappa>0$ ).

\section{Methods}

This section provides a brief account of the methods to investigate Problem A, namely, the existence problem of compact Clifford-Klein forms of symmetric spaces.

3.1. Generalized concept of properly discontinuous actions. Putting aside compactness for the moment, we concentrate on the condition that the double coset space $\Gamma \backslash G / H$ becomes a Clifford-Klein form of the symmetric space $G / H$ with emphasis on non-compact $H$ :

(a) The $\Gamma$-action on $G / H$ is fixed point free.

(b) The $\Gamma$-action on $G / H$ is properly discontinuous.

Under the condition (b), the condition (a) is fulfilled if $\Gamma$ is torsion free. Furthermore, if $\Gamma$ is finitely generated and linear then $\Gamma$ contains a torsion free subgroup of finite index ([Sel60]). Thus, the condition (a) is not very serious, provided the condition (b) is satisfied. In the case $G / H$ is a Riemannian symmetric space, the condition (b) is automatically satisfied for any discrete subgroup $\Gamma$ of $G$. However, for a non-Riemannian symmetric space $G / H$ where $H$ is not compact, the condition (b) is not always satisfied, and the resulting double coset space $\Gamma \backslash G / H$ may not be Hausdorff with respect to the quotient topology.

Thus, in the study of Problem A for non-Riemannian symmetric spaces, it is crucial to understand why the condition (b) may fail. 
It is in general a hard problem to find an 'efficient' criterion for the condition (b). The first breakthrough was done in [Ko88], where the strategy is summarized as:

Point 1) Forget the original assumption that $\Gamma$ is a discrete subgroup.

Point 2) Reformulate the problem inside the group $G$ so that $\Gamma$ and $H$ play a symmetric role in $G$, instead of the traditional approach that treats directly the $\Gamma$-action on the homogeneous space $G / H$.

Point 3) Reformulate the properly discontinuous condition in terms of finite dimensional representation theory.

In order to emphasize Point (1), we shall use the notation $L$ instead of $\Gamma$.

Based on this strategy, the criterion of proper actions (see Lemma 3.2.3) was proved under the assumption that both $L$ and $H$ are reductive subgroups of a reductive Lie group $G$, where Point (3) was pursued by using the standard theory of parabolic subalgebras of a reductive Lie algebra $\mathfrak{g}$.

The proof in [Ko89a] shows that even the group structure of $H$ and $L$ is not important. During his stay at the Institute for Advanced Study at Princeton invited by Armand Borel in 1991-1992, the first author studied the deformation of crystallographic groups for $G / H$ with non-compact $H$. This study led us to the following simple concept:

Definition 3.1.1 ([Ko96a, Definition 2.1.1]). Let $L$ and $H$ be subsets of a locally compact group $G$.

1) We say the pair $(L, H)$ is proper in $G$, denoted by $L \pitchfork H$, if $L \cap S H S$ is relatively compact for any compact set $S$ in $G$.

2) We say the pair $(L, H)$ is similar in $G$, denoted by $L \sim H$, if there exists a compact set $S$ in $G$ such that $L \subset S H S$ and $H \subset S L S$.

The point here is that we do not require $L$ and $H$ to be subgroups. However, our motivation of introducing $\pitchfork$ goes back to the following observation:

Lemma 3.1.2. Suppose $H$ is a closed subgroup of a locally compact group $G$, and $\Gamma$ is a discrete subgroup of $G$. Then the natural $\Gamma$-action on $G / H$ is properly discontinuous if and only if $\Gamma \pitchfork H$.

Remark 3.1.3. Lemma 3.1.2 can be generalized as follows: Suppose $L$ is a closed subgroup of $G$. Then the natural $L$-action on $G / H$ is proper in the sense of Palais [P61] if and only if $L \pitchfork H$. We note that a proper action of a discrete subgroup is properly discontinuous, and vice versa.

We recall from [Ko96a, Section 2] some basic properties of the relations $\sim$ and $\pitchfork:$ 
Lemma 3.1.4. Let $L, L^{\prime}$ and $H$ be subsets of a locally compact group $G$.

1) The relation $\sim$ is an equivalence relation.

2) If $L \sim L^{\prime}$, then $L \pitchfork H \Leftrightarrow L^{\prime} \pitchfork H$.

3) $L \pitchfork H \Longleftrightarrow H \pitchfork L$.

Remark 3.1.5. 1) If $H \sim G$, then $L \pitchfork H$ if and only if $L$ is compact.

2) If $H$ is compact, then $L \pitchfork H$ for any subset $L$ in $G$.

Remark 3.1.5 has the following three interesting consequences:

Proposition 3.1.6. Let $H$ be a closed subgroup of a locally compact group $G$.

1) Only a finite subgroup $\Gamma \subset G$ can act on $G / H$ properly discontinuously if $H \sim G$ (the Calabi-Markus phenomenon; see Theorem 3.2.5).

2) Any discrete subgroup $\Gamma \subset G$ acts on the homogeneous space $G / H$ properly discontinuously if $H$ is a compact subgroup of $G$.

3) Suppose $\Gamma$ is a cocompact discrete subgroup of $G$. Then the $\Gamma$-action on $G / H$ is properly discontinuous if and only if $H$ is compact.

The rest of this subsection collects some further basic properties of the relations $\pitchfork$ and $\sim$ together with another relation $\sim_{s}$, that will be used in Section 5.

Lemma 3.1.7. Let $G$ be a Euclidean space, and let $L$ and $H$ be closed cones of $G$. Then the following equivalences hold.

1) $L \pitchfork H \Longleftrightarrow L \cap H=\{0\}$.

2) $L \sim H \Longleftrightarrow L=H$.

Proof. 1) The implication $L \pitchfork H \Rightarrow L \cap H=\{0\}$ is obvious from Definition 3.1.1. Conversely, let us prove $L \not H \Rightarrow L \cap H \neq\{0\}$. We find sequences $\left\{l_{n}\right\} \subset$ $L,\left\{h_{n}\right\} \subset H$ and a positive number $M>0$ such that

$$
\sup _{n}\left\|l_{n}-h_{n}\right\| \leq M, \quad \lim _{n \rightarrow \infty}\left\|l_{n}\right\|=\infty .
$$

Taking a subsequence, if necessary, we may and do assume the limit $l:=\lim \frac{l_{n}}{\left\|l_{n}\right\|}$ exists. Since

$$
\left\|\frac{l_{n}}{\left\|l_{n}\right\|}-\frac{h_{n}}{\left\|l_{n}\right\|}\right\|=\frac{\left\|l_{n}-h_{n}\right\|}{\left\|l_{n}\right\|} \leq \frac{M}{\left\|l_{n}\right\|} \rightarrow 0 \quad(n \rightarrow \infty),
$$

we have $\lim \frac{h_{n}}{\left\|l_{n}\right\|}=l$. As both $H$ and $L$ are closed cones, we conclude $l \in L \cap H$. Hence, $L \cap H \neq\{0\}$.

2) Obviously, $L=H \Rightarrow L \sim H$. Let us prove $L \sim H \Rightarrow L=H$. We take a compact set $S$ such that $L \subset H+S$. For any $l \in L$ and any $n \in \mathbb{N}$, we have 
$n l \in L$, so that we find $h_{n} \in H$ and $s_{n} \in S$ satisfying $n l=h_{n}+s_{n}$. Thus we have

$$
\frac{n l-s_{n}}{n}=\frac{h_{n}}{n} \in H \text {. }
$$

Taking the limit as $n$ tends to $\infty$, we have $l \in H$ because $H$ is closed. This means $L \subset H$. Similarly, $H \subset L$. Thus, we have proved (2).

Now we introduce another relation $\sim_{s}$, which is far stronger than $\sim$. For actual applications, we shall need the case where $L$ and $H$ are subgroups.

Definition 3.1.8. Let $L$ and $H$ be subsets of a locally compact group $G$. We say the pair $(L, H)$ is (right) strongly similar in $G$, denoted by $L \sim_{s} H$, if there exist $g \in G$ and a compact set $S$ in $G$ such that $L \subset g H S$ and $H \subset g L S$.

Clearly, $\sim_{s}$ is an equivalence relation. The following lemma is an obvious consequence of the definition:

Lemma 3.1.9. Let $G$ be a locally compact group, and let $L \supset H$ be two closed subgroups. Then, $L / H$ is compact if and only if $L \sim_{s} H$.

However, the relation $\sim_{s}$ will be useful later, when there is no inclusive relation between $L$ and $H$. We pin down some basic properties of $\sim_{s}$ (and $\left.\sim, \pitchfork\right)$ :

Lemma 3.1.10. Let $L, L^{\prime}$ and $H$ be closed subgroups of a locally compact group $G$. Assume that $L \sim_{s} L^{\prime}$, then the following equivalence holds:

$$
L \backslash G / H \text { is compact } \Longleftrightarrow L^{\prime} \backslash G / H \text { is compact. }
$$

Lemma 3.1.11. Let $G_{1}$ be a locally compact group, and $G_{2}$ its closed subgroup. Suppose $L$ and $H$ are subgroups of $G_{2}$. Then,

1) $L \pitchfork H$ in $G_{1} \Rightarrow L \pitchfork H$ in $G_{2}$.

2) $L \sim H$ in $G_{2} \Rightarrow L \sim H$ in $G_{1}$.

3) $L \sim_{s} H$ in $G_{2} \Rightarrow L \sim_{s} H$ in $G_{1}$.

Lemma 3.1.12. Let $G$ be a locally compact group and $N$ a normal subgroup. We write the quotient map as $\varpi: G \longrightarrow G / N$. For two subsets $\bar{L}$ and $\bar{H}$ in $G / N$, we set $L:=\varpi^{-1}(\bar{L})$ and $H:=\varpi^{-1}(\bar{H})$. Then,

$$
L \sim_{s} H \text { in } G \Longleftrightarrow \bar{L} \sim_{s} \bar{H} \text { in } G / N .
$$

Remark 3.1.13. An analogous statement of Lemma 3.1.12 is true if we replace $\sim_{s}$ by $\sim$. However, an analogous statement for $\pitchfork$ is not true. In fact, $\bar{L} \pitchfork \bar{H}$ does not imply $L \pitchfork H$.

The proof of Lemmas 3.1.9, 3.1.10 and 3.1.11 are straightforward from the definition. Let us show Lemma 3.1.12. 
Proof of Lemma 3.1.12. $\Rightarrow$ ) Take $g \in G$ and a compact set $S$ in $G$ such that $L \subset g H S$ and $H \subset g L S$. Applying $\varpi$, we have $\bar{L} \subset \varpi(g) \bar{H} \varpi(S)$ and $\bar{H} \subset$ $\varpi(g) \bar{L} \varpi(S)$. Since $\varpi(S)$ is compact, $\bar{L} \sim_{s} \bar{H}$ in $G / N$.

$\Leftarrow)$ Take $\bar{g} \in G / N$ and a compact set $\bar{S}$ in $G / N$ such that $\bar{L} \subset \bar{g} \bar{H} \bar{S}$ and $\bar{H} \subset \bar{g} \bar{L} \bar{S}$. We take $g \in \varpi^{-1}(\bar{g})$ and a compact set $S$ in $G$ such that $\bar{S} \subset \varpi(S)$. Then,

$$
L=\varpi^{-1}(\bar{L}) \subset \varpi^{-1}(\varpi(g) \bar{H} \varpi(S))=g H S .
$$

Similarly, we have $H \subset g L S$. Thus, the implication ' $\Leftarrow$ ' has been proved.

3.2. Criterion of properness for reductive groups. This subsection explicates a criterion of $\pitchfork$ and $\sim$ for subsets of a linear reductive Lie group $G$. Our criterion is 'computable' by means of the Cartan projection, and will play a key role in the study of Clifford-Klein forms of non-Riemannian symmetric spaces $G / H$. An analogous result for the Cartan motion group will be given in Section 5 .

Let $G$ be a linear reductive Lie group, and $K$ a maximal compact subgroup. We write $\theta$ for the corresponding Cartan involution, and write $\mathfrak{g}=\mathfrak{k}+\mathfrak{p}$ for the Cartan decomposition of the Lie algebra $\mathfrak{g}$. Choose a maximal abelian subspace $\mathfrak{a}$ in $\mathfrak{p}$. The dimension of $\mathfrak{a}$ is independent of the choice, and is called the real rank of $G$, denoted by $\mathbb{R}$ - rank $G$. Then the Cartan decomposition

$$
G=K \exp (\mathfrak{p})=K \exp (\mathfrak{a}) K
$$

has the following property: any element $g \in G$ can be written as $g=k_{1} e^{X} k_{2}$ for some $k_{1}, k_{2} \in K$, and the element $X$ of $\mathfrak{a}$ is uniquely determined up to the conjugation by the Weyl group $W_{G}:=N_{G}(\mathfrak{a}) / Z_{G}(\mathfrak{a})$. The correspondence $g \mapsto X$ induces the Cartan projection

$$
\nu: G \rightarrow \mathfrak{a} / W_{G}
$$

For a subset $L$ of $G$, the image of the Cartan projection is described as a $W_{G^{-}}$ invariant subset as follows:

$$
\begin{aligned}
\mathfrak{a}(L) & :=\left\{X \in \mathfrak{a}: k_{1} e^{X} k_{2} \in L \quad \text { for some } k_{1}, k_{2} \in K\right\} \\
& =\left\{X \in \mathfrak{a}: e^{X} \in K L K\right\} .
\end{aligned}
$$

Here is a criterion of properness $\pitchfork$ and similarity $\sim$ for reductive Lie groups.

Lemma 3.2.1. For any subsets $L$ and $H$ in a linear reductive Lie group $G$, we have

1) $L \pitchfork H$ in $G \Longleftrightarrow \mathfrak{a}(L) \pitchfork \mathfrak{a}(H)$ in $\mathfrak{a}$.

2) $L \sim H$ in $G \Longleftrightarrow \mathfrak{a}(L) \sim \mathfrak{a}(H)$ in $\mathfrak{a}$.

Here, we regard $\mathfrak{a} \simeq \mathbb{R}^{n}$ as an abelian Lie group in the right-hand side. 
Proof. See [Ko96a, Theorem 1.1] for a proof in the same circle of ideas of [Ko89a]. See also [Bn96, Théorème 5.2] for a proof where $G$ is a reductive group over a local field.

Remark 3.2.2. There is mysterious similarity between the criterion (1) of Lemma 3.2.1 for proper actions and the criterion for the non-existence of continuous spectrum in the irreducible decomposition of unitary representations ([Ko98c]). See $[$ Ko05, Section $4 \mathrm{D}]$ for details.

In this paper, by a reductive subgroup, we shall mean a closed subgroup $H$ which has the following two properties:

(a) $H$ has at most finitely many connected components.

(b) $H$ is stable under some Cartan involution of $G$.

Then $H$ itself is a reductive Lie group. We say the resulting homogeneous space $G / H$ is of reductive type. A reductive (or semisimple) symmetric space is a typical example.

Suppose $H$ is a reductive subgroup of $G$, and we take a maximal split abelian subspace $\mathfrak{a}^{\prime}$ of the Lie algebra $\mathfrak{h}$ of $H$. Then, there is $g \in G$ such that $\operatorname{Ad}(g) \mathfrak{a}^{\prime}$ is contained in the fixed maximal split abelian subspace $\mathfrak{a}$ for $G$. We set

$$
\mathfrak{a}_{H}:=\operatorname{Ad}(g) \mathfrak{a}^{\prime} .
$$

Then $\mathfrak{a}_{H}$ is well-defined up to the action of the Weyl group $W_{G}$. If $H$ is stable under the fixed Cartan involution $\theta$, it is not hard to see

$$
\mathfrak{a}(H)=W_{G} \cdot \mathfrak{a}_{H} .
$$

For such $\theta$-stable subgroups $L$ and $H$, the above criterion of properness (Lemma 3.2.1) is of a much simpler form.

Lemma 3.2.3 ([Ko89a, Theorem 4.1]). Suppose $L$ and $H$ are $\theta$-stable subgroups, with finitely many connected components. Then

$$
L \pitchfork H \text { in } G \Longleftrightarrow \mathfrak{a}(L) \cap \mathfrak{a}(H)=\{0\} .
$$

Example 3.2.4. Let $G$ be an indefinite orthogonal group $O(p, q)(p \geq q)$. We set $H_{i}:=E_{p+i, i}+E_{i, p+i} \in \mathfrak{g}(1 \leq i \leq q)$. Then, the $q$-dimensional vector space

$$
\mathfrak{a}:=\bigoplus_{i=1}^{q} \mathbb{R} H_{i}
$$

is a maximal split abelian subspace. For a subgroup $H=O(r, s)$, where $r \leq p$ and $s \leq q$, the subset $\mathfrak{a}(H)$ is of the form:

$$
\mathfrak{a}(H)=\bigcup_{1 \leq i_{1}<\cdots<i_{\min (r, s)} \leq q}\left(\bigoplus_{k=1}^{\min (r, s)} \mathbb{R} H_{i_{k}}\right) .
$$


Calabi and Markus proved in [CaM62] that there does not exist an infinite discontinuous group for the Lorentz symmetric space $O(n+1,1) / O(n, 1)$. Named after their result, the non-existence of infinite discontinuous group is sometimes referred to as the Calabi-Markus phenomenon. The Calabi-Markus phenomenon in the reductive case has been studied by Calabi and Markus [CaM62], Wolf [Wo62, 64], Kulkarni [Ku81] and Kobayashi [Ko89a], and it has been settled completely in terms of the rank condition as follows. This criterion is obtained as an application of Lemma 3.2.1.

Theorem 3.2.5 ([Ko89a]). Let $G / H$ be a homogeneous space of reductive type. Then the following two conditions are equivalent:

(i) Any discontinuous group for $G / H$ is finite.

(ii) $\mathbb{R}-\operatorname{rank} G=\mathbb{R}-\operatorname{rank} H$.

Sketch of the proof. The condition $\mathbb{R}$ - $\operatorname{rank} H=\mathbb{R}$ - $\operatorname{rank} G$ is equivalent to $\mathfrak{a}(H)=$ $\mathfrak{a}$, and then equivalent to $H \sim G$ by Lemma 3.2.1. Then, only a finite group acts properly discontinuously on $G / H$ (Proposition 3.1.6). Conversely, if $\mathbb{R}-\operatorname{rank} G>$ $\mathbb{R}$-rank $H$, we can take $X \in \mathfrak{a} \backslash \mathfrak{a}(H)$. Then, $\exp (\mathbb{Z} X)$ acts on $G / H$ properly discontinuously and freely because of Lemma 3.2.3 and $\mathbb{R} X \cap \mathfrak{a}(H)=\{0\}$.

Remark 3.2.6. If $M$ is a compact topological space, then clearly only a finite group can act properly discontinuously on $M$. The homogeneous space $G / H$ satisfying the rank condition (ii) behaves as if $G / H$ were a compact space with respect to the transformation group $G$.

Geometrically, a homogeneous space of reductive type is a good example of pseudo-Riemannian manifold.

Proposition 3.2.7. A homogeneous space $G / H$ of reductive type has a $G$-invar -iant pseudo-Riemannian structure of signature

$$
(d(G)-d(H), \operatorname{dim} G-\operatorname{dim} H-d(G)+d(H)) .
$$

For a Lie group $G$, we define

$$
d(G):=\operatorname{dim} G-\operatorname{dim} K
$$

where $K$ is a maximal compact subgroup of $G$. Then, $d(G)$ is well-defined because any two maximal compact subgroups are conjugate to each other, and may be regarded as the 'non-compact dimension' of $G$. For a reductive Lie group $G$ with a Cartan decomposition $\mathfrak{g}=\mathfrak{k}+\mathfrak{p}$, we note

$$
d(G)=\operatorname{dim} \mathfrak{p} .
$$

Here are some examples.

\begin{tabular}{c|c|c|c|c|c|c}
$G$ & $G L(n, \mathbb{R})$ & $S O(p, q)$ & $S U(p, q)$ & $S p(p, q)$ & $S p(n, \mathbb{R})$ & $G L(n, \mathbb{C})$ \\
\hline$d(G)$ & $\frac{n(n+1)}{2}$ & $p q$ & $2 p q$ & $4 p q$ & $n^{2}+n$ & $n^{2}$
\end{tabular}


Proof of Proposition 3.2.7. Without loss of generality, we shall assume that $H$ is $\theta$-stable.

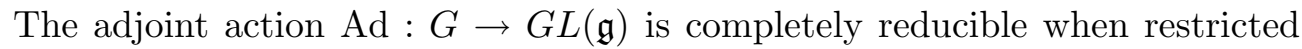
to $H$. Then, we take $\mathfrak{q}$ to be the $H$-invariant complementary subspace of $\mathfrak{h}$ in $\mathfrak{g}$. Since the decomposition $\mathfrak{g}=\mathfrak{h}+\mathfrak{q}$ is $\theta$-stable, we have

$$
\mathfrak{g}=\mathfrak{k}+\mathfrak{p}=(\mathfrak{k} \cap \mathfrak{h})+(\mathfrak{k} \cap \mathfrak{q})+(\mathfrak{p} \cap \mathfrak{h})+(\mathfrak{p} \cap \mathfrak{q}) .
$$

We fix a $G$-invariant symmetric bilinear form $B$ on $\mathfrak{g}$ such that $\left.B\right|_{\mathfrak{k} \times \mathfrak{k}}$ is negative definite, $\left.B\right|_{\mathfrak{p} \times \mathfrak{p}}$ is positive definite, and $\mathfrak{k}$ is orthogonal to $\mathfrak{p}$ with respect to $B$ (e.g. the Killing form if $\mathfrak{g}$ is semisimple). Then the restriction of $B$ to $\mathfrak{q} \times \mathfrak{q}$ is an $H$ invariant non-degenerate symmetric bilinear form of signature $(\operatorname{dim}(\mathfrak{q} \cap \mathfrak{p}), \operatorname{dim}(\mathfrak{q} \cap$ $\mathfrak{k})$ ), which equals (3.2.5). Then, the left $G$-translation of this bilinear form gives rise to a $G$-invariant pseudo-Riemannian structure on $G / H$.

3.3. Construction of compact Clifford-Klein forms. So far, we have discussed properly discontinuous actions by putting aside the compactness condition. Building on the criterion of properness (Lemma 3.2.1 or Lemma 3.2.3), we shall study the existence problem of compact Clifford-Klein forms (Problem A).

A cocompact discrete subgroup $\Gamma$ of $G$ cannot act properly discontinuously on the homogeneous space $G / H$ unless $H$ is compact (Proposition 3.1.6(3)). This means that a crystallographic group for $G / H$ must be 'smaller' than a uniform lattice of $G$. Instead of $G$ itself, a uniform lattice of a subgroup $L$ may be a candidate of a crystallographic group for $G / H$. This idea leads us to the following simple method that constructs a compact Clifford-Klein form of $G / H$ with $H$ non-compact.

Theorem 3.3.1 ([Ko89a, Theorem 4.7]). Let $G$ be a Lie group, and $H$ its closed subgroup. Then the homogeneous space $G / H$ admits a compact Clifford-Klein form if there is a subgroup $L$ with the following three properties:

(3.3.1)(a) $L \pitchfork H$ in $G$ (see Definition 3.1.1),

(3.3.1)(b) $L \backslash G / H$ is compact in the quotient topology,

(3.3.1)(c) L has a cocompact, torsion free discrete subgroup $\Gamma$.

Sketch of the proof. From (a) and (c), $\Gamma$ acts on $G / H$ properly discontinuously and freely. Then, $\Gamma \backslash G / H$ is a compact Clifford-Klein form of $G / H$ by (b) and (c).

Remark 3.3.2. If $L$ is a linear group, then one can drop the torsion free assumption in (c). In fact, a finitely generated linear group $\Gamma$ contains a torsion free subgroup $\Gamma^{\prime}$ of finite index by a result of Selberg [Sel60]. 
In the reductive case, we have a much explicit formulation of Theorem 3.3.1. In what follows, the role of $L$ and $H$ will be symmetric. This symmetric role goes back to Lemma 3.1.4(3).

Theorem 3.3.3 ([Ko89a]). Let $G$ be a reductive linear Lie group, and let $L$ and $H$ be $\theta$-stable subgroups with finitely many connected components. If $L$ and $H$ satisfy the following two properties, then both $G / H$ and $G / L$ admit compact Clifford-Klein forms.

(3.3.3)(a) $\mathfrak{a}(L) \cap \mathfrak{a}(H)=0$,

$(3.3 .3)(\mathrm{b}) \quad d(L)+d(H)=d(G)$.

Sketch of the proof. The condition (3.3.1)(a) follows from the criterion of $L \pitchfork$ $H$ (see Lemma 3.2.3), while the condition (3.3.1)(c) always holds for reductive linear Lie groups by Borel's result (see Theorem 1.1.1). The remaining condition (3.3.1)(b) follows from the numerical criterion given in Lemma 3.3.4.

Lemma 3.3.4 ([Ko89a, Theorem 4.7], see also [IW04, Theorem 9.1]). Let $G$ be a linear reductive Lie group. Suppose that connected closed subgroups $L$ and $H$ satisfy $L \pitchfork H$ in $G$. Then we have:

1) $d(L)+d(H) \leq d(G)$.

2) $d(L)+d(H)=d(G)$ if and only if $L \backslash G / H$ is compact.

Remark 3.3.5. Clearly, Lemma 3.3.4 still holds if $L$ and $H$ have finitely many connected components.

Remark 3.3.6. It might be illuminative to see that Lemma 3.3.4 applied to $L=$ $\{e\}$ means:

$$
G / H \text { is compact } \Longleftrightarrow d(G)=d(H) \text {. }
$$

Corollary 3.3.7. The following semisimple symmetric spaces $G / H$ have compact Clifford-Klein forms, as the triple $(G, H, L)$ fulfills the conditions (3.3.3)(a) and (b). Here, $n=1,2, \ldots$. 


\begin{tabular}{c|c|c} 
& $G / H$ & $L$ \\
\hline 1 & $S U(2,2 n) / S p(1, n)$ & $U(1,2 n)$ \\
2 & $S U(2,2 n) / U(1,2 n)$ & $S p(1, n)$ \\
3 & $S O(2,2 n) / U(1, n)$ & $S O(1,2 n)$ \\
4 & $S O(2,2 n) / S O(1,2 n)$ & $U(1, n)$ \\
5 & $S O(4,4 n) / S O(3,4 n)$ & $S p(1, n)$ \\
6 & $S O(4,4) / S O(4,1) \times S O(3)$ & $S p i n(4,3)$ \\
7 & $S O(4,3) / S O(4,1) \times S O(2)$ & $G_{2(2)}$ \\
8 & $S O(8,8) / S O(7,8)$ & $S p i n(1,8)$ \\
9 & $S O(8, \mathbb{C}) / S O(7, \mathbb{C})$ & $S p i n(1,7)$ \\
10 & $S O(8, \mathbb{C}) / S O(7,1)$ & $S p i n(7, \mathbb{C})$ \\
11 & $S O^{*}(8) / U(3,1)$ & $S p i n(1,6)$ \\
12 & $S O^{*}(8) / S O^{*}(6) \times S O^{*}(2)$ & $\operatorname{Spin}(1,6)$
\end{tabular}

Table 3.3

In Table 3.3, the role of $L$ and $H$ is symmetric in the cases 1 and 2, and in the cases 3 and 4 (see Theorem 3.3.3).

Kulkarni [Ku81] proved the existence of a compact Clifford-Klein form of $G / H$ in the cases 4 and 5 . The cases 1, 2 and 3 were proved in [Ko89a]. The cases 6 and 7 are given in [Ko92a, Corollary 3]. The cases 11 and 12 were given in [Ko96b, Examples 5.15 and 5.16]. See also [Ko96b, Corollary 4.7] for some non-symmetric examples such as $G / H=S O(4,4 n) / S p(1, n)(n=1,2, \ldots)$ that admit compact Clifford-Klein forms. We shall give a detailed proof of the cases 8, 9 and 10 in Section 4.

Remark 3.3.8. By using local isomorphisms of Lie groups $S O^{*}(8) \approx S O(2,6)$ and $S O^{*}(2) \times S O^{*}(6) \approx U(1,3)$, we see that $S O^{*}(8) / U(3,1)$ (the case 11) and $S O^{*}(8) / S O^{*}(6) \times S O^{*}(2)$ (the case 12) are infinitesimally isomorphic to $S O(2,6) / U(1,3)$ (the case 2$)$.

Remark 3.3.9. Suppose $L$ and $H$ are $\theta$-stable connected subgroups of a connected reductive Lie group $G$. If $\mathfrak{l}+\mathfrak{h}=\mathfrak{g}$, or equivalently, if $\mathfrak{l}_{\mathbb{C}}+\mathfrak{h}_{\mathbb{C}}=\mathfrak{g}_{\mathbb{C}}$, then the inclusion $L \subset G$ induces a natural diffeomorphism: $L / L \cap H \simeq G / H$ ([Ko94a, Lemma 5.1]). If $L \cap H$ is furthermore compact and if $L$ is linear, then all the assumptions of Theorem 3.3.3 are satisfied, and consequently, $G / H$ admits a compact Clifford-Klein form. See [Ko94a, Example 5.2] for some list of the triple $(G, H, L)$ of real reductive linear Lie groups satisfying $\mathfrak{l}+\mathfrak{h}=\mathfrak{g}$. This gives an alternative proof of Corollary 3.3.7. 
To the best of our knowledge, all of the semisimple symmetric spaces that have been proved to admit compact Clifford-Klein forms so far are infinitesimally isomorphic to a direct product of those $G / H$ in Table 3.3, Riemannian symmetric spaces and reductive group manifolds. We venture to make the following:

Conjecture 3.3.10. If the homogeneous space $G / H$ of reductive type admits a compact Clifford-Klein form, then there exists a reductive subgroup $L$ satisfying the assumptions of Theorem 3.3.1.

Remark 3.3.11. Conjecture 3.3.10 does not assert the following (false) statement: $A$ crystallographic group for $G / H$ is contained in a reductive subgroup $L$ satisfying the assumptions of Theorem 3.3.1. The deformation argument in [G85], [Ko98a] and $[\mathrm{SaO0}]$ gives counterexamples of this (too strong) statement.

Remark 3.3.12. A 'tangential' analog of Conjecture 3.3.10 will be formulated and proved in Section 5 (see Theorem 5.3.1).

3.4. Obstruction I - Calabi-Markus phenomenon. In Section 3.3, we have given a sufficient condition for symmetric spaces to admit compact CliffordKlein forms. Conversely, Sections 3.4 to 3.6 will explain some obstructions for symmetric spaces to have compact forms. By using these obstructions, we shall find a number of symmetric spaces without cocompact discontinuous groups. These examples support Conjecture 3.3.10.

Loosely, one might think of the reason why some $G / H$ cannot have a compact Clifford-Klein form as follows:

I) $G / H$ does not admit a 'large' discontinuous group.

II) $H$ does not attain its maximum of 'non-compact dimension'.

These ideas will be formulated into theorems in Sections 3.4 and 3.5, respectively. Such formulations are made possible because we know an explicit criterion for $\pitchfork$ (see Lemma 3.2.1).

We start with a first obstruction obtained by the criterion (see Theorem 3.2.5) of the Calabi-Markus phenomenon ([Ko89a]):

Theorem 3.4.1. Suppose $G$ is a reductive Lie group, and $H$ is its reductive subgroup with equal real rank. Then, $G / H$ does not admit a compact CliffordKlein form, unless $G / H$ itself is compact, or equivalently, if $d(G)>d(H)$.

Applying Theorem 3.4.1 to the 'space form problem' (see Section 2.5), we obtain immediately:

Corollary 3.4.2 ([Wo62]). The symmetric space $G / H=O(p+1, q) / O(p, q)$ does not admit compact Clifford-Klein forms if $p \geq q>0$. 
Proof. As $\mathbb{R}-\operatorname{rank} G=\min (p+1, q)$ and $\mathbb{R}-\operatorname{rank} H=\min (p, q)$, they coincide if $p \geq q$. Furthermore, $G / H$ is not compact because $d(G)-d(H)=(p+1) q-p q=$ $q>0$. Thus, Corollary 3.4.2 follows from Theorem 3.4.1.

Remark 3.4.3. The case $q=1$ in Corollary 3.4.2 was proved by Calabi-Markus [CaM62]. See Section 4.1 for the condition on $(p, q)$ for which $G / H$ is known to have compact Clifford-Klein forms.

The criterion for $\pitchfork$ (Lemma 3.2.1) strengthens Theorem 3.4.1 if we consider non-abelian discontinuous groups instead of abelian discontinuous groups as follows.

Let $\Sigma \equiv \Sigma(\mathfrak{g}, \mathfrak{a})$ be the restricted root system, and let $W_{G}$ be its Weyl group. We fix a positive system $\Sigma^{+} \equiv \Sigma^{+}(\mathfrak{g}, \mathfrak{a})$, and denote by $\mathfrak{a}_{+}$the corresponding dominant chamber, and write $w_{0}$ for the longest element of $W_{G}$. We set

$$
\mathfrak{b}_{+}:=\left\{X \in \mathfrak{a}_{+}: w_{0} X=-X\right\} .
$$

Suppose $H$ is a reductive subgroup of $G$, and we write $\mathfrak{a}_{H} \subset \mathfrak{a}$ as in (3.2.2).

Theorem 3.4.4 ([Bn96]). Let $G / H$ be a homogeneous space of reductive type. Then the following two conditions are equivalent:

(i) Any discontinuous group for $G / H$ is virtually abelian.

(ii) $\mathfrak{b}_{+} \subset w \mathfrak{a}_{H}$ for some $w \in W_{G}$.

Here, an abstract group is said to be virtually abelian if it contains an abelian subgroup of finite index.

Theorem 3.4.5 ([Bn96, Corollary 7.6]). Suppose $G$ is a reductive Lie group, and $H$ its reductive subgroup. If $\mathfrak{b}_{+} \subset w \mathfrak{a}_{H}$ for some $w \in W_{G}$, then $G / H$ does not admit a compact Clifford-Klein form, unless $G / H$ itself is compact.

Theorem 3.2.5 concerns with infinite discontinuous groups, whereas Theorem 3.4.4 concerns with non-abelian discontinuous groups. Here is a comparison:

$$
\begin{array}{ccc}
\text { Theorem } 3.2 .5(\mathrm{i}) & \Rightarrow & \text { Theorem } 3.4 .4(\mathrm{i}) \\
\mathbb{1} & \mathbb{1} \\
\text { Theorem 3.2.5(ii) } & \Rightarrow & \text { Theorem 3.4.4(ii) }
\end{array}
$$

We note that if $\mathfrak{b}_{+}=\mathfrak{a}_{+}$, then all of the above four conditions are equivalent. On the other hand, if $\mathfrak{b}_{+} \subsetneq \mathfrak{a}_{+}$, then Theorem 3.4.5 is stronger than Theorem 3.4.1. We remark that $\mathfrak{b}_{+} \subsetneq \mathfrak{a}_{+}$occurs when $\Sigma$ is of type $A_{n}(n \geq 2), D_{2 m+1}$ or $E_{6}$.

Example 3.4.6. 1) Suppose $\Sigma(\mathfrak{g}, \mathfrak{a})$ is of type $A_{n-1}$. We choose a positive system $\Sigma^{+}$to be $\left\{e_{i}-e_{j}: 1 \leq i<j \leq n\right\}$ so that $\mathfrak{a}_{+}=\left\{\left(x_{1}, \ldots, x_{n}\right) \in \mathbb{R}^{n}: x_{1} \geq \cdots \geq\right.$ 
$\left.x_{n}, \sum x_{j}=0\right\}$. Then $w_{0} \neq-1$ if and only if $n \geq 3$, and we have

$$
\mathfrak{b}_{+}= \begin{cases}\left\{\left(x_{1}, \ldots, x_{m},-x_{m}, \ldots,-x_{1}\right): x_{1} \geq \cdots \geq x_{m} \geq 0\right\} & (n=2 m), \\ \left\{\left(x_{1}, \ldots, x_{m}, 0,-x_{m}, \ldots,-x_{1}\right): x_{1} \geq \cdots \geq x_{m} \geq 0\right\} & (n=2 m+1) .\end{cases}
$$

2) Suppose $\Sigma(\mathfrak{g}, \mathfrak{a})$ is of type $D_{n}$. We choose $\Sigma^{+}(\mathfrak{g}, \mathfrak{a})=\left\{e_{i} \pm e_{j}, e_{k}: 1 \leq i<j \leq\right.$ $n, 1 \leq k \leq n\}$ so that $\mathfrak{a}_{+}=\left\{\left(x_{1}, \ldots, x_{n}\right) \in \mathbb{R}^{n}: x_{1} \geq \cdots \geq x_{n-1} \geq\left|x_{n}\right|\right\}$. Then $w_{0} \neq-1$ if and only if $n$ is odd, and

$$
\mathfrak{b}_{+}= \begin{cases}\left\{\left(x_{1}, \ldots, x_{n-1}, 0\right): x_{1} \geq \cdots \geq x_{n-1} \geq 0\right\} & (n \text { is odd }), \\ \mathfrak{a}_{+} & (n \text { is even }) .\end{cases}
$$

Corollary 3.4.7. The following symmetric spaces $G / H$ do not admit compact Clifford-Klein forms.

\begin{tabular}{l|c} 
& $G / H$ \\
\hline 1 & $S L(2 n, \mathbb{R}) / S p(n, \mathbb{R})$ \\
2 & $S L(2 n+1, \mathbb{R}) / S O(n, n+1)$ \\
3 & $S O(2 n+1,2 n+1) / S O(2 n, 2 n+1)$ \\
4 & $S L(2 n, \mathbb{C}) / S p(n, \mathbb{C})$ \\
5 & $S O(4 n+2, \mathbb{C}) / S O(4 n+1, \mathbb{C})$ \\
6 & $E_{6, \mathbb{C}} / F_{4, \mathbb{C}}$
\end{tabular}

Table 3.4

3.5. Obstruction II - maximality of non-compactness. If two subgroups $H$ and $L$ satisfy $H \sim L$, then any discontinuous group for $G / H$ is also a discontinuous group for $G / L$. Then, the comparison of two homogeneous spaces $G / H$ and $G / L$ gives rise to a second necessary condition for the existence of compact Clifford-Klein forms for $G / H$ :

Theorem 3.5.1 ([Ko92b, Theorem 1.5]). Let $G / H$ be a homogeneous space of reductive type. If there exists a reductive subgroup $L$ in $G$ such that $L \sim H$ and $d(L)>d(H)$, then $G / H$ does not admit compact Clifford-Klein forms.

Remark 3.5.2. It is readily seen that the subgroup $L$ in this Theorem becomes an obstruction for the existence of the subgroup $L$ in Theorem 3.3.1. In this sense, Theorem 3.5.1 affords evidence for Conjecture 3.3.10.

A key step of proving Theorem 3.5.1 is to formulate a discrete analog of Lemma 3.3.4 in terms of the virtual cohomological dimension $v c d(\Gamma)$ of an abstract group $\Gamma$ as follows:

Lemma 3.5.3 ([Ko89a]). Let $G / H$ be a homogeneous space of reductive type. If a discrete subgroup $\Gamma \subset G$ satisfies $\Gamma \pitchfork H$, then we have: 
1) $\operatorname{vcd}(\Gamma)+d(H) \leq d(G)$.

2) $v c d(\Gamma)+d(H)=d(G)$ if and only if $\Gamma \backslash G / H$ is compact.

Remark 3.5.4. A special case of Lemma 3.5.3(2) with $H=\{e\}$ asserts that

$$
v c d(\Gamma)=d(G)
$$

if $\Gamma$ is a cocompact discrete subgroup of $G$. This result is due to Serre [Ser71].

Proof of Theorem 3.5.1 (sketch). Let $\Gamma$ be a uniform lattice for $G / H$, then we have $\operatorname{vcd}(\Gamma)+d(H)=d(G)$. On the other hand, we have $\Gamma \pitchfork L$, thus $\operatorname{vcd}(\Gamma)+$ $d(L) \leq d(G)$. This contradicts to $d(L)>d(H)$.

Remark 3.5.5. As one can see from the proof, Theorem 3.5.1 can be strengthened by replacing the assumption $L \sim H$ by $\mathfrak{a}(L) \subset \mathfrak{a}(H)$.

Remark 3.5.6. Taking $L$ to be $G$ in Theorem 3.5.1, we obtain an alternative proof of Theorem 3.4.1.

The following symmetric space $G / H$ is a good test case, as it contains many parameters. We note that $G / H$ becomes a pseudo-Riemannian space form if $i=0$ and $k=1$.

Corollary 3.5.7 ([Ko92b, Example 1.7]). Let G/H be a pseudo-Riemannian Grassmannian manifold

$$
O(i+j, k+l) / O(i, k) \times O(j, l) .
$$

Without loss of generality, we may and do assume $i \leq j, k, l$. We assume that neither $H$ nor $G / H$ is compact, that is, $j, k, l>0$. If $G / H$ admits compact Clifford-Klein forms, then $i=0$ and $0<l \leq j-k$.

Proof. Assume $G / H$ admits compact Clifford-Klein forms. From Theorem 3.4.1, we have $\mathbb{R}$ - $\operatorname{rank} H<\mathbb{R}$ - $\operatorname{rank} G$, that is,

$$
i+\min (j, l)<\min (i+j, k+l) .
$$

In particular, $l<j$. On the other hand, we define two subgroups $L_{1}$ and $L_{2}$ by

$$
\begin{aligned}
& L_{1}:=O(i+j, i+l) \subset O(i+j, k+l), \\
& L_{2}:=O(i+l, k+l) \subset O(i+j, k+l) .
\end{aligned}
$$

Then, we have $\mathfrak{a}(H)=\mathfrak{a}\left(L_{1}\right)=\mathfrak{a}\left(L_{2}\right)$ (see Example 3.2.4). Thus $H \sim L_{1} \sim L_{2}$ in $G$ by Lemma 3.2.1. Now, applying Theorem 3.5.1, we have the following two inequalities

$$
\begin{aligned}
& d(H)=i k+j l \geq d\left(L_{1}\right)=(i+l)(k+l), \\
& d(H)=i k+j l \geq d\left(L_{2}\right)=(i+j)(i+l) .
\end{aligned}
$$

Combining with $l<j$, we conclude that $i=0$ and $l \leq j-k$. 
Remark 3.5.8. The same results as Corollary 3.5.7 hold if we consider indefinite unitary groups over $\mathbb{C}$ or $\mathbb{H}$ instead of $\mathbb{R}$, namely, if we replace $O(p, q)$ by $U(p, q)$ or $S p(p, q)$.

Corollary 3.5.9 ([Ko92b, Table 4.4] and [Ko96a, Table 5.18]). The following symmetric spaces $G / H$ do not admit compact Clifford-Klein forms as the pair $(G / H, L)$ satisfies the assumptions of Theorem 3.5.1 (or Remark 3.5.5). In the table, we always assume $n=p+q$. We denote by $\lfloor m\rfloor$ the maximal integer that does not exceed $n$.

\begin{tabular}{|c|c|c|}
\hline & $G / H$ & $\operatorname{Lie}(L)$ \\
\hline 1 & $S L(2 n, \mathbb{R}) / S O(n, n)$ & $\mathfrak{s p}(n, \mathbb{R})$ \\
\hline 2 & $S U^{*}(2 n) / S O^{*}(2 n)$ & $\mathfrak{s p}\left(\left\lfloor\frac{n}{2}\right\rfloor, n-\left\lfloor\frac{n}{2}\right\rfloor\right)$ \\
\hline 3 & $S U(2 n, 2 n) / S O^{*}(4 n)$ & $\mathfrak{s p}(n, n)$ \\
\hline 4 & $S p(2 n, \mathbb{R}) / U(n, n)$ & $\mathfrak{s p}(n, \mathbb{C})$ \\
\hline 5 & $S O(2 n, 2 n) / S O(2 n, \mathbb{C})$ & $\mathfrak{u}(n, n)$ \\
\hline 6 & $S O^{*}(2 n) / S O^{*}(2 p) \times S O^{*}(2 q) \quad(p, q>1)$ & $\mathfrak{s o}^{*}(2)+\mathfrak{s o}^{*}(2 n-2)$ \\
\hline 7 & $S L(n, \mathbb{C}) / S O(n, \mathbb{C})$ & $\mathfrak{u}\left(\left\lfloor\frac{n}{2}\right\rfloor, n-\left\lfloor\frac{n}{2}\right\rfloor\right)$ \\
\hline 8 & $S O(n, \mathbb{C}) / S O(p, \mathbb{C}) \times S O(q, \mathbb{C}) \quad(p, q>1)$ & $\mathfrak{s o}(n-1, \mathbb{C})$ \\
\hline 9 & $S O^{*}(2 n) / U(p, q)$ & $\begin{array}{c}\mathfrak{s o}^{*}(2 r) \\
r=\min (n, 2 p+1,2 q+1)\end{array}$ \\
\hline 10 & $S L(2 n, \mathbb{C}) / S U(n, n)$ & $\mathfrak{s p}(n, \mathbb{C})$ \\
\hline 11 & $S p(n, \mathbb{R}) / S p(p, \mathbb{R}) \times S p(q, \mathbb{R})$ & $\mathfrak{s p}(n, \mathbb{R})$ \\
\hline 12 & $E_{6(6)} / S p(4, \mathbb{R})$ & $\mathfrak{f}_{4(4)}$ \\
\hline 13 & $E_{6(2)} / S U(4,2) \times S U(2)$ & $\mathfrak{s o}^{*}(10)+\sqrt{-1} \mathbb{R}$ \\
\hline 14 & $E_{7(7)} / S U(4,4)$ & $\mathfrak{e}_{6(2)}+\sqrt{-1} \mathbb{R}$ \\
\hline 15 & $E_{7(7)} / S U^{*}(8)$ & $\mathfrak{s o}^{*}(12)+\mathfrak{s u}(2)$ \\
\hline 16 & $E_{7(-5)} / S U(6,2)$ & $\mathfrak{e}_{6(-14)}+\sqrt{-1} \mathbb{R}$ \\
\hline 17 & $E_{7(-25)} / S U(6,2)$ & $\mathfrak{e}_{6(-14)}+\sqrt{-1} \mathbb{R}$ \\
\hline 18 & $E_{8(8)} / S O^{*}(16)$ & $\mathfrak{e}_{7(-5)}+\mathfrak{s u}(2)$ \\
\hline 19 & $E_{6, \mathbb{C}} / S p(4, \mathbb{C})$ & $\mathfrak{f}_{4, \mathbb{C}}$ \\
\hline
\end{tabular}

Table 3.5

Some remarks on Table 3.5 are in order. 
1) In the case 6 , there exists a compact Clifford-Klein form for $(p, q)=(1,3)$ and $(3,1)$.

2) In the cases 6 and 8 with $p q$ even, and in the case 11, there is an alternative proof by using Theorem 3.4.1.

3) Taking this opportunity, we correct typographical errors in [Ko96a, Table 5.18]; the pairs $(S L(2 n, \mathbb{C}), S U(p, q))$ and $(S L(2 n, \mathbb{R}), S O(p, q))$ there should read $(S L(n, \mathbb{C}), S U(p, q))$ and $(S L(n, \mathbb{R}), S O(p, q))$, respectively.

In what follows, we omit an explanation of the technical terms "associated pair" and "basic in $\epsilon$-family", but we just indicate how to find $L$ systematically in most of the cases in Table 3.5.

Lemma 3.5.10 ([Ko92b, Theorem 1.4]). A reductive symmetric space $G / H$ admits a compact Clifford-Klein form only if its associated symmetric pair $\left(G, H^{a}\right)$ is basic in the $\epsilon$-family.

3.6. Obstruction III - compatibility of three compact spaces. A CliffordKlein form $\Gamma \backslash G / H$ inherits local geometric structure from $G / H$ through the covering map $G / H \rightarrow \Gamma \backslash G / H$. Such geometric structures may become some constraints on the topology of Clifford-Klein forms.

Another constraint is derived from the fiber bundle structure $G / H \rightarrow K / H \cap$ $K$. The compatibility of these constraints yields a necessary condition for the existence of compact Clifford-Klein form, formulated as follows:

Theorem 3.6.1 ([Ko89a, Proposition 4.10]). Suppose G is a reductive Lie group, and $H$ is its reductive subgroup. If $\operatorname{rank} G=\operatorname{rank} H$ and $\operatorname{rank} K>\operatorname{rank} H \cap K$, then $G / H$ does not admit a compact Clifford-Klein form.

Let $G_{\mathbb{C}}$ be a complexification of $G$, and let $G_{U}$ be a compact real form of $G_{\mathbb{C}}$ (for example, $G_{U}=S U(n)$ if $G=S L(n, \mathbb{R})$ ). Likewise $H_{U}$ for $H$. Thus, we have the following setting:

$$
\begin{array}{cccccc}
G & \subset & G_{\mathbb{C}} & \supset & G_{U} \\
\cup & & \cup & & \cup \\
H & \subset & H_{\mathbb{C}} & \supset & H_{U}
\end{array}
$$

The compact homogeneous space $G_{U} / H_{U}$ plays a role of a 'real form' of $G_{\mathbb{C}} / H_{\mathbb{C}}$. The idea of analytic continuation leads us to:

Lemma 3.6.2 ([KoO90]). There is a canonical $\mathbb{C}$-algebra homomorphism between de Rham cohomology groups:

$$
\eta: H^{*}\left(G_{U} / H_{U} ; \mathbb{C}\right) \rightarrow H^{*}(\Gamma \backslash G / H ; \mathbb{C}) .
$$

If $\Gamma \backslash G / H$ is compact, then $\eta$ is injective. Furthermore, $\eta$ sends the characteristic classes for $G_{U} / H_{U}$ to the corresponding ones for $\Gamma \backslash G / H$. 
Remark 3.6.3. If $H=K$, a maximal compact subgroup of $G$, then there exists a compact Clifford-Klein form $\Gamma \backslash G / K$ (see Theorem 1.1.1). In this case, the image of $\eta$ is isomorphic to the $(\mathfrak{g}, K)$-cohomology group $H^{*}(\mathfrak{g}, K ; \mathbb{C})$ via the Matsushima-Murakami isomorphism ([BoW00]):

$$
H^{*}(\Gamma \backslash G / H ; \mathbb{C}) \simeq \bigoplus_{\pi \in \hat{G}} \operatorname{Hom}_{G}\left(\pi, L^{2}(\Gamma \backslash G)\right) \otimes H^{*}\left(\mathfrak{g}, K ; \pi_{K}\right),
$$

where $\pi_{K}$ is the $(\mathfrak{g}, K)$-module of an irreducible unitary representation $\pi$ of $G$.

Proof of Theorem 3.6.1 (sketch). The idea is to compare three compact spaces $G_{U} / H_{U}, K / H \cap K$ and $\Gamma \backslash G / H$. The Euler number $\chi\left(G_{U} / H_{U}\right) \neq 0$ if $\operatorname{rank} G=$ rank $H$ by a theorem of Hopf and Samelson [HoS41]. On the other hand, we can show $\chi(\Gamma \backslash G / H)=0$ by using the fiber bundle structure $G / H \rightarrow K / H \cap K$, provided $\operatorname{rank} K>\operatorname{rank} K \cap H$. By Lemma 3.6.2 $\chi(\Gamma \backslash G / H)=\eta\left(\chi\left(G_{U} / H_{U}\right)\right)$. Therefore, $\eta$ cannot be injective. Hence, $\Gamma \backslash G / H$ cannot be compact.

Here are some examples of Theorem 3.6.1:

Corollary 3.6.4. A semisimple symmetric space $\operatorname{Sp}(2 n, \mathbb{R}) / \operatorname{Sp}(n, \mathbb{C})$ does not admit a compact Clifford-Klein form.

Proof. Both the ranks of $S p(2 n, \mathbb{R})$ and $S p(n, \mathbb{C})$ are $2 n$, while the ranks of their maximal compact subgroups $U(2 n)$ and $S p(n)$ are $2 n$ and $n$, respectively. Therefore, Corollary follows from Theorem 3.6.1.

Corollary 3.6.5. If $j k l$ is odd, an indefinite Grassmannian manifold

$$
G / H=O(j, k+l) / O(k) \times O(j, l)
$$

does not admit a compact Clifford-Klein form.

Proof. The condition $\operatorname{rank} G=\operatorname{rank} H$ amounts to

$$
\left\lfloor\frac{j+k+l}{2}\right\rfloor=\left\lfloor\frac{k}{2}\right\rfloor+\left\lfloor\frac{j+l}{2}\right\rfloor .
$$

On the other hand, the condition $\operatorname{rank} K>\operatorname{rank} H \cap K$ amounts to

$$
\left\lfloor\frac{j}{2}\right\rfloor+\left\lfloor\frac{k}{2}\right\rfloor+\left\lfloor\frac{l}{2}\right\rfloor>\left\lfloor\frac{j}{2}\right\rfloor+\left\lfloor\frac{k+l}{2}\right\rfloor .
$$

This holds if and only if $k l$ is odd. Under the assumption $k l$ is odd, (3.6.1) is equivalent to that $j$ is odd. Thus, Corollary follows from Theorem 3.6.1.

Remark 3.6.6. Theorem 1.1.6 implies particularly that there do not exist compact Clifford-Klein forms of the tangential symmetric space $G_{\theta} / H_{\theta}$ for $G / H=O(p+$ $1, q) / O(p, q)$ if $p>0$ and $q$ is odd. This parity condition may be regarded as a 'tangential' analog of Corollary 3.6.5 with $k=1, l=p$ and $j=q$. 


\section{SPACE FORM PROBleM}

By the "space form problem" we mean an (unsolved) problem whether or not there exists a compact complete pseudo-Riemannian manifold of general signature $(p, q)$ with constant sectional curvature. This section provides a proof of the 'if' part of Conjecture 2.5.1 to the space form problem, together with a proof of the 'if' part of Conjecture 2.4.2 for complex version of space form problem (namely the cases 8 and 9 of Theorem 2.2.1). An algebraic machinery for this is the Clifford algebra associated to indefinite quadratic form that we develop in Sections 4.3-4.8. This leads us to another family of finitely many homogeneous spaces that admit compact Clifford-Klein forms arisen from the spin representation (see Theorem 4.2.1). We also complete the proof of the remaining case of Theorem 2.2.1, namely, the case 10 in Table 2.2.

4.1. Space form conjecture. This subsection summarizes the current status of the 'space form conjecture' (Conjecture 2.5.1). First, we equip $\mathbb{R}^{p+q}$ with the pseudo-Riemannian metric

$$
d s^{2}=d x_{1}^{2}+\cdots+d x_{p}^{2}-d x_{p+1}^{2}-\cdots-d x_{p+q}^{2} .
$$

The resulting pseudo-Riemannian manifold, denoted by $\mathbb{R}^{p, q}$, is a flat space form with signature $(p, q)$.

Next, let $Q_{p, q}$ be a quadratic form on $\mathbb{R}^{p+q}$ defined by

$$
Q_{p, q}(x):=x_{1}^{2}+\cdots+x_{p}^{2}-x_{p+1}^{2}-\cdots-x_{p+q}^{2} .
$$

We induce a pseudo-Riemannian structure on the hypersurface

$$
X(p, q):=\left\{x \in \mathbb{R}^{p+1, q}: Q_{p+1, q}(x)=1\right\}
$$

from $\mathbb{R}^{p+1, q}$. Then $X(p, q)$ is a space form of signature $(p, q)$ with sectional curvature +1 . It is simply connected if $p \geq 2$.

Remark 4.1.1. Note that $X(p, q)$ has signature $(q, p)$ with sectional curvature -1 , if we replace the pseudo-Riemannian metric $g$ by $-g$.

Here are some well-known cases.

1) $\mathbb{R}^{3,1}$ is the Minkowski space.

2) $X(p, 0)$ is the $p$-sphere $S^{p}$.

3) $X(0, q)$ is a hyperbolic space.

4) $X(p, 1)$ is a de Sitter space.

5) $X(1, q)$ is an anti-de Sitter space.

The indefinite orthogonal group

$$
O(p, q)=\left\{g \in G L(p+q, \mathbb{R}): Q_{p, q}(g x)=Q_{p, q}(x) \text { for any } x \in \mathbb{R}^{p+q}\right\}
$$


acts transitively on $X(p, q)$, so that $X(p, q)$ is represented as the homogeneous space $O(p+1, q) / O(p, q)$. We notice that $O(p+1, q) / O(p, q)$ is a semisimple symmetric space of rank one. Then, it is plausible that Problem A for this symmetric space has the following solution:

Conjecture 4.1.2. The symmetric space $M=O(p+1, q) / O(p, q)$ admits compact Clifford-Klein forms if and only if $(p, q)$ is in the list below:

\begin{tabular}{c||c|c|c|c|c}
$p$ & $\mathbb{N}$ & 0 & 1 & 3 & 7 \\
\hline$q$ & 0 & $\mathbb{N}$ & $2 \mathbb{N}$ & $4 \mathbb{N}$ & 8
\end{tabular}

We note:

- $p=0$ or $q=0 \quad M$ is a Riemannian symmetric space.

- $p=1,3,7 \quad M$ is a pseudo-Riemannian symmetric space.

Remark 4.1.3. Conjecture 4.1.2 is equivalent to Conjecture 2.5.1. In fact, in the case $\kappa=0$, Conjecture 2.5.1 is true because $\mathbb{R}^{p, q}$ has a uniform lattice $\mathbb{Z}^{p+q}$. In the case $\kappa<0$, Conjecture 2.5.1 is reduced to the case $\kappa>0$ as we saw in Remark 4.1.1. Conjecture 2.5.1 and Conjecture 4.1.2 are also true in the case $p=1$ and $\kappa>0$ : compact forms exist by Theorem 2.2.1 if $q$ is even, while compact forms do not exist by Corollary 3.6.5 if $q$ is odd.

For the remaining case where $p \geq 2$ and $\kappa>0$, the universal covering manifold of any complete space form of signature $(p, q)$ with sectional curvature $\kappa$ is isometrically (up to a positive scalar multiplication of metric) diffeomorphic to the pseudo-Riemannian symmetric space $X(p, q) \simeq O(p+1, q) / O(p, q)$. Thus, Conjecture 2.5.1 is equivalent to Conjecture 4.1.2.

As for the 'only if' part of Conjecture 4.1.2, to the best of our knowledge, the following is all the cases that the non-existence of compact space forms has been proved so far.

(a) $p \geq q>0 \quad$ (see Corollary 3.4.2).

(b) $p+1=q$ is odd (see Corollary 3.4.7).

(c) $p q$ is odd (see Corollary 3.6.5).

Conversely, the 'if' part of the Conjecture 4.1.2 is true. Here is a brief account of each case.

1) $q=0 ; \quad M=S^{p}$ itself is compact.

2) $p=0 ; \quad M$ is a Riemannian symmetric space and has a compact Clifford-Klein form by Theorem 1.1.1. 
For the remaining three pseudo-Riemannian cases, let us see $G / H$ admits compact Clifford-Klein forms by applying Theorem 3.3.3.

3) $(p, q)=(1,2 n) ; \quad G=O(2,2 n), \quad H=O(1,2 n)$.

Take a subgroup $L$ to be $U(1, n)$. By choosing a suitable basis, we identify $\mathfrak{a} \simeq \mathbb{R}^{2}$, on which the Weyl group $W_{G} \simeq \mathfrak{S}_{2} \ltimes(\mathbb{Z} / 2 \mathbb{Z})^{2}$ acts by permutation and change of signatures of coordinates. Then one finds

$$
\mathfrak{a}(H)=W_{G} \cdot \mathbb{R}\left(\begin{array}{l}
1 \\
0
\end{array}\right), \quad \mathfrak{a}(L)=W_{G} \cdot \mathbb{R}\left(\begin{array}{l}
1 \\
1
\end{array}\right),
$$

and therefore $\mathfrak{a}(L) \cap \mathfrak{a}(H)=\{0\}$. On the other hand, we have $d(L)+d(H)=$ $2 n+2 n=4 n=d(G)$. Now, it follows from Theorem 3.3.3 that a uniform lattice of $L$ becomes a cocompact discontinuous group for $G / H$.

4) $(p, q)=(3,4 n) ; \quad G=O(4,4 n), \quad H=O(3,4 n)$.

Take $L=S p(1, n)$. Similarly to the previous case, one finds

$$
\mathfrak{a}=\mathbb{R}^{4}, \quad W_{G} \simeq \mathfrak{S}_{4} \ltimes(\mathbb{Z} / 2 \mathbb{Z})^{4}, \quad \mathfrak{a}(H)=W_{G} \cdot \mathbb{R}\left(\begin{array}{l}
1 \\
1 \\
1 \\
0
\end{array}\right), \quad \mathfrak{a}(L)=W_{G} \cdot \mathbb{R}\left(\begin{array}{l}
1 \\
1 \\
1 \\
1
\end{array}\right),
$$

and so $\mathfrak{a}(L) \cap \mathfrak{a}(H)=\{0\}$. Furthermore, $d(L)+d(H)=12 n+4 n=16 n=d(G)$. Hence, $G / H$ admits a compact Clifford-Klein form by Theorem 3.3.3.

The rest of this section is devoted to proving Theorem 1.1.5, namely, the existence of a compact Clifford-Klein form for the last case:

5) $(p, q)=(7,8) ; \quad G=O(8,8), \quad H=O(7,8)$.

We shall take a subgroup $L$ to be $\operatorname{Spin}(1,8)$. We need to embed $\operatorname{Spin}(1,8)$ into $G$ and then to find $\mathfrak{a}(L) \subset \mathfrak{a}$. For this purpose, we study the Clifford algebra $C(p, q)$ for the indefinite quadratic form $Q_{p, q}$ of signature $(p, q)$. This will be carried out in Sections 4.3 to 4.8 based on an unpublished note [Ko94b], in the way that we need for the proof of Theorems 1.1.3 and 1.1.5.

4.2. Compact Clifford-Klein forms. As was stated in Theorem 2.2.1, there exist compact Clifford-Klein forms for the symmetric spaces $O(8, \mathbb{C}) / O(7, \mathbb{C})$ and $O(8,8) / O(7,8)$. We shall provide a unified proof of these results. That is, the following theorem exhibits a family of finitely many homogeneous spaces $G / H$ that admit uniform lattices arisen from the spin representation. Then the homogeneous space $O(8, \mathbb{C}) / O(7, \mathbb{C})$ and $O(8,8) / O(7,8)$ appear corresponding to $q=7$ and 8 , respectively in Theorem 4.2 .1 below.

This family of homogeneous spaces will be treated systematically based on Clifford algebras of indefinite quadratic forms. 
Theorem 4.2.1. The triples of Lie groups $(G, H, L)=(G(1, q), H(1, q)$, Spin $(1, q))$ $(1 \leq q \leq 8)$ satisfy the conditions (3.3.3)(a) and (b), and therefore, both of the homogeneous spaces $G / H$ and $G / L$ admit compact Clifford-Klein forms.

\begin{tabular}{|c|ccc|cccccc|}
\hline$q$ & $G$ & $H$ & $L$ & $W$ & $d(G)$ & $=$ & $d(L)$ & + & $d(H)$ \\
\hline 1 & $G L(1, \mathbb{R})$ & $\{e\}$ & $S p i n(1,1)$ & $\mathbb{R}$ & 1 & $=$ & 1 & + & 0 \\
2 & $S p(1, \mathbb{R})$ & $\{e\}$ & $S p i n(1,2)$ & $\mathbb{R}^{2}$ & 2 & $=$ & 2 & + & 0 \\
3 & $S p(1, \mathbb{C})$ & $\{e\}$ & $S p i n(1,3)$ & $\mathbb{C}^{2}$ & 3 & $=$ & 3 & + & 0 \\
4 & $S p(1,1)$ & $S p(1)$ & $S p i n(1,4)$ & $\mathbb{H}^{2}$ & 4 & $=$ & 4 & + & 0 \\
5 & $G L(2, \mathbb{H})$ & $G L(1, \mathbb{H})$ & $S p i n(1,5)$ & $\mathbb{H}^{2}$ & 6 & $=$ & 5 & + & 1 \\
6 & $O^{*}(8)$ & $O^{*}(6)$ & $S p i n(1,6)$ & $\mathbb{H}^{4}$ & 12 & $=$ & 6 & + & 6 \\
7 & $O(8, \mathbb{C})$ & $O(7, \mathbb{C})$ & $S p i n(1,7)$ & $\mathbb{C}^{8}$ & 28 & $=$ & 7 & + & 21 \\
8 & $O(8,8)$ & $O(7,8)$ & $S p i n(1,8)$ & $\mathbb{R}^{16}$ & 64 & $=$ & 8 & + & 56 \\
\hline
\end{tabular}

Table 4.2

Here, $G(p, q)$ is a Lie group contained in the Clifford algebra $C(p, q)$ associated to the quadratic form of indefinite signature $(p, q)$ (see Definition 4.3.1). The general structural theorem of $G(p, q)$ will be stated in Proposition 4.4.4. In particular, $G(p, q)$ acts on the vector space $W$ as a natural representation, and $H$ is defined as the isotropy subgroup at $e_{1}$, where $\left\{e_{i}\right\}$ is the standard basis of $W$.

Remark 4.2.2. 1) For $q \leq 4$, Theorem 4.2.1 is just a special case of Borel's theorem (see Theorem 1.1.1), because $H$ is compact then. This is also the case if $q=5$ because $H$ is locally isomorphic to $\mathbb{R} \times S O(3)$.

2) The cases $6 \leq q \leq 8$ are remarkable as both of $H$ and $L$ are non-compact. Theorems 1.1.3 and 1.1.5 correspond to the cases $q=7$ and $q=8$ of Theorem 4.2.1, respectively. Corollary 3.3.7 (12) (up to a compact factor) corresponds to the case $q=6$ of Theorem 4.2.1.

3) In light of the case $q=7$, we can prove that the triple $(O(8, \mathbb{C}), O(1,7)$, Spin $(7, \mathbb{C}))$ satisfies the conditions $(3.3 .3)(\mathrm{a})$ and (b) so that the symmetric space $O(8, \mathbb{C}) / O(7, \mathbb{C})$ admits a compact Clifford-Klein form (see Theorem 4.10.1). This can be justified by an outer automorphism of $\mathfrak{o}(8, \mathbb{C})$. This can be also proved by using [Ko94b]. However, we shall give another proof based on Clifford algebras in Section 4.10.

4) For $q \geq 9$, the condition (3.3.3)(a) holds, but the condition (3.3.3)(b) fails. It is likely that $G / H$ does not admit a compact Clifford-Klein form if $q \geq 9$. For example, $G / H=G L(16, \mathbb{R}) / G L(15, \mathbb{R})$ for $q=9$. We note that Conjecture 3.3 .10 implies that $G L(16, \mathbb{R}) / G L(15, \mathbb{R})$ does not admit compact Clifford-Klein forms. But even in this case, this is still an unsolved problem (see [Ko02] for the list 
of various results in the literature about the (non-)existence of compact CliffordKlein forms of non-symmetric spaces $G L(n, \mathbb{F}) / G L(m, \mathbb{F})(n>m ; \mathbb{F}=\mathbb{R}, \mathbb{C}$ or $\mathbb{H})$.

Outline of proof. The condition (3.3.3)(b) is clearly satisfied from the Table 4.2. We shall prove the condition (3.3.3)(a) in a more general setting in Section 4.9 (see Proposition 4.9.2), after a general theory of Clifford algebras. The last statement of Theorem 4.2.1 is a direct consequence of Theorem 3.3.3.

4.3. Clifford algebra associated to an indefinite quadratic form. This subsection elucidates the structure of the Clifford algebra associated to the indefinite quadratic form $Q(a)=a_{1}^{2}+\cdots+a_{p}^{2}-a_{p+1}^{2}-\cdots-a_{p+q}^{2}$ of signature $(p, q)$. In contrast to the case that $Q$ is negative (or positive) definite (e.g. [ABS64]), there are more periodicity theorems and abundant structures, in particular, through the shift $(p, q) \mapsto(p+1, q+1)$ and the flip $(p, q) \mapsto(q+1, p-1)$.

We have tried to make the exposition here self-contained as far as possible.

Let $E=\mathbb{R}^{p, q}$ be the vector space $\mathbb{R}^{p+q}$ equipped with the quadratic form $Q(a)$ for $a=\sum_{j=1}^{p+q} a_{j} e_{j} \in \mathbb{R}^{p+q}$, where $\left\{e_{j}\right\}$ is the standard basis of $\mathbb{R}^{p+q}$. Let $T(E)$ be the tensor algebra over $\mathbb{R}$, and let $I(Q)$ be the two sided ideal generated by the element $a \otimes a-Q(a) \cdot 1$ in $T(E)$. The quotient algebra $T(E) / I(Q)$ is called the Clifford algebra, and will be denoted by $C(Q)$ or $C(p, q)$. We define $i_{Q}: E \rightarrow$ $C(p, q)$ to be the canonical map by the composition $E \rightarrow T(E) \rightarrow C(p, q)$. Then $i_{Q}$ is injective, and we shall regard $E$ as a subspace of $C(p, q)$.

The Clifford algebra $C(p, q)$ is the $\mathbb{R}$-algebra generated by the unit 1 and the symbols $e_{j}$ subject to the relations:

(4.3.1) $e_{j}^{2}=1(1 \leq j \leq p), e_{j}^{2}=-1(p+1 \leq j \leq p+q), e_{i} e_{j}+e_{j} e_{i}=0(i \neq j)$.

We state the universality of the $\mathbb{R}$-algebra $C(p, q)$ in the form that will be frequently used later: Let $\phi: E \mapsto A$ be a linear map of $E$ into an $\mathbb{R}$-algebra $A$ with unit 1 satisfying the following conditions (4.3.2):

$$
\begin{aligned}
\phi\left(e_{j}\right)^{2}= & 1(1 \leq j \leq p), \quad \phi\left(e_{j}\right)^{2}=-1(p+1 \leq j \leq p+q), \\
& \phi\left(e_{i}\right) \phi\left(e_{j}\right)+\phi\left(e_{j}\right) \phi\left(e_{i}\right)=0(i \neq j) .
\end{aligned}
$$

Then there exists a unique $\mathbb{R}$-algebra homomorphism $\tilde{\phi}: C(p, q) \rightarrow A$, such that $\tilde{\phi} \circ i_{Q}=\phi . \tilde{\phi}$ automatically satisfies $\tilde{\phi}(1)=1$. We refer to $\tilde{\phi}$ as the extension of $\phi$, and shall use the same notation $\phi$ instead of $\tilde{\phi}$. In particular, if $\phi$ is a linear isomorphism $\phi: E \rightarrow E(\subset C(p, q))$ that preserves the quadratic form $Q$, then $\phi$ is extended to an algebra isomorphism $\phi: C(p, q) \stackrel{\sim}{\rightarrow} C(p, q)$.

The Clifford algebra is naturally a $\mathbb{Z}_{2}$-graded algebra

$$
C(p, q)=C_{\text {odd }}(p, q) \oplus C_{\text {even }}(p, q),
$$


as is defined to be the image in $C(p, q)$ of $T(E)=\bigoplus_{j=0}^{\infty} T^{2 j+1}(E)+\bigoplus_{j=0}^{\infty} T^{2 j}(E)$.

Further, we define an anti-ring homomorphism

$$
C(p, q) \rightarrow C(p, q), \quad a \mapsto{ }^{t} a
$$

as the extension of the identity map $E \rightarrow E$. If $a$ is of the form $a=a_{1} \cdots a_{s} \in$ $C(p, q)$ for some $a_{i} \in E$, then ${ }^{t} a=a_{s} \cdots a_{1}$.

Definition 4.3.1. We define the subset $C(p, q)_{\text {mon }}$ and the groups $G(p, q)$ and $\operatorname{Spin}(p, q)$ in $C(p, q)$ as follows:

$$
\begin{aligned}
G(p, q) & :=\left\{a \in C_{\text {even }}(p, q):{ }^{t} a a=1\right\}, \\
C(p, q)_{\text {mon }} & :=\bigcup_{s=0}^{\infty}\left\{ \pm a_{1} \cdots a_{s}: a_{i} \in E\right\}, \\
\operatorname{Spin}(p, q) & :=G(p, q) \cap C(p, q)_{\text {mon }} .
\end{aligned}
$$

We pin down an obvious inclusive relation:

$$
\operatorname{Spin}(p, q){ }^{C}{ }_{G(p, q)} \subset^{C(p, q)_{\text {mon }}} \subset{ }^{C(p, q)}
$$

Remark 4.3.2. The signature \pm in the definition of $C(p, q)_{\text {mon }}$ is not very important. In fact, an easy computation shows $C(p, q)_{\text {mon }}=\bigcup_{s=0}^{\infty}\left\{a_{1} \cdots a_{s}: a_{i} \in E\right\}$ if $(p, q) \neq(0,0),(1,0)$.

The quadratic form $Q$ on $E$ defines an inner product $\langle$,$\rangle of signature (p, q)$ on $E$ by

$$
\langle a, b\rangle:=\frac{1}{2}(Q(a+b)-Q(a)-Q(b))=\frac{1}{2}(a b+b a)
$$

for $a, b \in E$. We note $\langle a, a\rangle=Q(a)$.

Take $v \in E$ such that $Q(v) \neq 0$ and $x \in \operatorname{Spin}(p, q)$. We set

$$
\begin{aligned}
\tau_{v}(a) & :=\frac{-1}{Q(v)} v a v \\
\rho(x)(a) & :=x a^{t} x
\end{aligned}
$$

for $a \in E$. It turns out that both $\tau_{v}(a)$ and $\rho(x)(a)$ belong to $E$. In fact, both $\tau_{v}$ and $\rho(x)$ belong to the indefinite orthogonal group $O(p, q)$ as is seen in the following:

Proposition 4.3.3. 1) $\tau_{v}(a) \in E$ for $a \in E$. More precisely, $\tau_{v}$ is a reflection in $E$ with respect to the inner product $\langle\cdot, \cdot\rangle$ given by

$$
\tau_{v}(a)=a-2 \frac{\langle a, v\rangle}{Q(v)} v .
$$

In particular, $\tau_{v} \in O(p, q)$ and $\operatorname{det}\left(\tau_{v}\right)=-1$. 
2) $\rho(x)=\tau_{x_{1}} \cdots \tau_{x_{2 n}}$ for $x=x_{1} \cdots x_{2 n} \in \operatorname{Spin}(p, q)$.

3) If $x \in \operatorname{Spin}(p, q)$, then $\rho(x)(a) \in E$ for any $a \in E$ and $\rho(x) \in S O(p, q)$.

4) $\rho$ defines a surjective group homomorphism $\operatorname{Spin}(p, q) \rightarrow S O(p, q)$ with $\operatorname{Ker} \rho=\{ \pm 1\}$.

Proof. Use $\langle a, v\rangle=\frac{1}{2}(a v+v a)$ for (1). (2) and (3) are obvious. The proof of the statement (4) parallels to that of the well-known case $q=0$.

Later, we shall use frequently the following lemma for specific choice of $v$.

Lemma 4.3.4. The reflection $\tau_{v}: E \rightarrow E$ extends to the $\mathbb{R}$-algebra involutive isomorphism $\tau_{v}: C(p, q) \stackrel{\sim}{\rightarrow} C(p, q)$.

4.4. Clifford algebra. This subsection summarizes structural results of the Clifford algebra $C(p, q)$ and the group $G(p, q)$ (see Definition 4.3.1) associated to the quadratic form $Q$ of arbitrary signature $(p, q)$. The proof will be given in Sections 4.6 and 4.8 , respectively.

For $\mathbb{F}=\mathbb{R}, \mathbb{C}$ and $\mathbb{H}$, we denote by $M(n, \mathbb{F})$ the $\mathbb{R}$-algebra $(\mathbb{C}$-algebra if $\mathbb{F}=\mathbb{C})$ of $n$ by $n$ matrices whose entries are in the field $\mathbb{F}$.

Proposition 4.4.1. For $p, q \geq 0$, we define $\alpha \in\{0,1,2,3\}$ as in the table below and set $n=2^{(p+q-\alpha) / 2}$. Then, the Clifford algebra $C(p, q)$ is isomorphic to one of the following algebras:

\begin{tabular}{c|c||c}
$C(p, q)$ & $\alpha$ & $p-q-1 \bmod 8$ \\
\hline$M(n, \mathbb{R}) \oplus M(n, \mathbb{R})$ & 1 & 0 \\
$M(n, \mathbb{R})$ & 0 & \pm 1 \\
$M(n, \mathbb{C})$ & 1 & \pm 2 \\
$M(n, \mathbb{H})$ & 2 & \pm 3 \\
$M(n, \mathbb{H}) \oplus M(n, \mathbb{H})$ & 3 & 4
\end{tabular}

We note that $\alpha$ depends only on $p-q \bmod 8$. The Clifford algebra $C(p, q)$ carries a $\mathbb{C}$-algebra structure if $p-q-1 \equiv \pm 2 \bmod 8$ (see Remark 4.5.2).

In an obvious sense, $C(p, q)$ is of period $(8,0)$ and $(0,8)$, and of $(1,1)$. By Proposition 4.4.1, we have the following table of $C(p, q)$ for $p, q \leq 8$. 


\begin{tabular}{|c|c|c|c|c|c|c|c|c|c|}
\hline$p{ }^{q}$ & 0 & 1 & 2 & 3 & 4 & 5 & 6 & 7 & 8 \\
\hline 0 & $\mathbb{R}$ & $\mathbb{C}$ & $\mathbb{H}$ & $\mathbb{H}^{2}$ & $\mathbb{H}(2)$ & $\mathbb{C}(4)$ & $\mathbb{R}(8)$ & $\mathbb{R}(8)^{2}$ & $\mathbb{R}(16)$ \\
\hline 1 & $\mathbb{R}^{2}$ & $\mathbb{R}(2)$ & $\mathbb{C}(2)$ & $\mathbb{H}(2)$ & $\mathbb{H}(2)^{2}$ & $\mathbb{H}(4)$ & $\mathbb{C}(8)$ & $\mathbb{R}(16)$ & $\mathbb{R}(16)^{2}$ \\
\hline 2 & $\mathbb{R}(2)$ & $\mathbb{R}(2)^{2}$ & $\mathbb{R}(4)$ & $\mathbb{C}(4)$ & $\mathbb{H}(4)$ & $\mathbb{H}(4)^{2}$ & $\mathbb{H}(8)$ & $\mathbb{C}(16)$ & $\mathbb{R}(32)$ \\
\hline 3 & $\mathbb{C}(2)$ & $\mathbb{R}(4)$ & $\mathbb{R}(4)^{2}$ & $\mathbb{R}(8)$ & $\mathbb{C}(8)$ & $\mathbb{H}(8)$ & $\mathbb{H}(8)^{2}$ & $\mathbb{H}(16)$ & $\mathbb{C}(32)$ \\
\hline 4 & $\mathbb{H}(2)$ & $\mathbb{C}(4)$ & $\mathbb{R}(8)$ & $\mathbb{R}(8)^{2}$ & $\mathbb{R}(16)$ & $\mathbb{C}(16)$ & $\mathbb{H}(16)$ & $\mathbb{H}(16)^{2}$ & $\mathbb{H}(32)$ \\
\hline 5 & $\mathbb{H}(2)^{2}$ & $\mathbb{H}(4)$ & $\mathbb{C}(8)$ & $\mathbb{R}(16)$ & $\mathbb{R}(16)^{2}$ & $\mathbb{R}(32)$ & $\mathbb{C}(32)$ & $\mathbb{H}(32)$ & $\mathbb{H}(32)^{2}$ \\
\hline 6 & $\mathbb{H}(4)$ & $\mathbb{H}(4)^{2}$ & $\mathbb{H}(8)$ & $\mathbb{C}(16)$ & $\mathbb{R}(32)$ & $\mathbb{R}(32)^{2}$ & $\mathbb{R}(64)$ & $\mathbb{C}(64)$ & $\mathbb{H}(64)$ \\
\hline 7 & $\mathbb{C}(8)$ & $\mathbb{H}(8)$ & $\mathbb{H}(8)^{2}$ & $\mathbb{H}(16)$ & $\mathbb{C}(32)$ & $\mathbb{R}(64)$ & $\mathbb{R}(64)^{2}$ & $\mathbb{R}(128)$ & $\mathbb{C}(128)$ \\
\hline 8 & $\mathbb{R}(16)$ & $\mathbb{C}(16)$ & $\mathbb{H}(16)$ & $\mathbb{H}(16)^{2}$ & $\mathbb{H}(32)$ & $\mathbb{C}(64)$ & $\mathbb{R}(128)$ & $\mathbb{R}(128)^{2}$ & $\mathbb{R}(256)$ \\
\hline
\end{tabular}

Table 4.4.1

Here, we have abbreviated $M(n, \mathbb{F})$ to $\mathbb{F}(n)$, and $M(n, \mathbb{F}) \oplus M(n, \mathbb{F})$ to $\mathbb{F}(n)^{2}$ for $\mathbb{F}=\mathbb{R}, \mathbb{C}$ or $\mathbb{H}$.

Remark 4.4.2. In the case $p=0$ or $q=0$, Table 4.4.1 is the same with [ABS64, Table 1], where their $C_{k}$ corresponds to our $C(0, k)$ and likewise $C_{k}^{\prime}$ to $C(k, 0)$.

A key lemma to Proposition 4.4.1 is the following (see Section 4.6 for the proof):

Lemma 4.4.3. We have two families of isomorphisms of Clifford algebra $C(p, q)$ :

$$
\begin{array}{lll}
C(p, q) \simeq C(q+r, p-r) & \text { for } r \equiv 1 & \bmod 4, \\
C(p, q) \simeq C(p+r, q-r) & \text { for } r \equiv 0 & \bmod 4 .
\end{array}
$$

Next, we determine the group structure of $G(p, q)$ (see Definition 4.3.1):

Proposition 4.4.4. For $p, q \geq 1$, the group $G(p, q)$ is isomorphic to one of the following classical Lie groups. Here, $n=2^{(p+q-\alpha) / 2}$ and $\alpha \in\{3,4,5,6\}$ are given according to $p-q \bmod 8$ as in the table. 


\begin{tabular}{|c|c|c|c|c|}
\hline$G(p, q)$ & $C_{\text {even }}(p, q)$ & $\alpha$ & $p-q \bmod 8$ & $p+q \quad \bmod 8$ \\
\hline $\begin{array}{c}O(n, n)^{2} \\
G L(2 n, \mathbb{R}) \\
S p(n, \mathbb{R})^{2}\end{array}$ & $M(2 n, \mathbb{R})^{2}$ & 4 & 0 & $\begin{array}{c}0 \\
\pm 2 \\
4\end{array}$ \\
\hline $\begin{array}{c}O(n, n) \\
S p(n, \mathbb{R})\end{array}$ & $M(2 n, \mathbb{R})$ & 3 & \pm 1 & $\begin{array}{l} \pm 1 \\
\pm 3\end{array}$ \\
\hline $\begin{array}{l}O(2 n, \mathbb{C}) \\
U(n, n) \\
S p(n, \mathbb{C})\end{array}$ & $M(2 n, \mathbb{C})$ & 4 & \pm 2 & $\begin{array}{c}0 \\
\pm 2 \\
4\end{array}$ \\
\hline $\begin{array}{c}O^{*}(4 n) \\
S p(n, n)\end{array}$ & $M(2 n, \mathbb{H})$ & 5 & \pm 3 & $\begin{array}{l} \pm 1 \\
\pm 3\end{array}$ \\
\hline $\begin{array}{c}O^{*}(4 n)^{2} \\
G L(2 n, \mathbb{H}) \\
S p(n, n)^{2}\end{array}$ & $M(2 n, \mathbb{H})^{2}$ & 6 & 4 & $\begin{array}{c}0 \\
\pm 2 \\
4\end{array}$ \\
\hline
\end{tabular}

In the above table, $n=\frac{1}{2}$ if $(p, q)=(1,1)$.

As is observed in Proposition 4.4.4, the type of $G(p, q)$ depends only on $p-q$ $\bmod 8$ and $p+q \bmod 8$. The following table exhibits the "basic pattern" of the group $G(p, q)$.

\begin{tabular}{c|ccccc}
\multicolumn{5}{c}{$G(p, q)$} & for $p \pm q \quad \bmod 8$ \\
$\begin{array}{c}5+q \\
p-q\end{array}$ & 0 & \pm 1 & \pm 2 & \pm 3 & 4 \\
\hline 0 & $O(n, n)^{2}$ & & $G L(2 n, \mathbb{R})$ & & $S p(n, \mathbb{R})^{2}$ \\
\pm 1 & & $O(n, n)$ & & $S p(n, \mathbb{R})$ & \\
\pm 2 & $O(2 n, \mathbb{C})$ & & $U(n, n)$ & & $S p(n, \mathbb{C})$ \\
\pm 3 & & $O^{*}(4 n)$ & & $S p(n, n)$ & \\
4 & $O^{*}(4 n)^{2}$ & & $G L(2 n, \mathbb{H})$ & & $S p(n, n)^{2}$
\end{tabular}

Table 4.4.2

For the convenience of the reader, we present the following table of the group $G(p, q)$ for $p, q \leq 8$ by adding the easier cases $p=0$ and $q=0$. 
$G(p, q) \quad(p, q \leq 8)$

\begin{tabular}{|c|c|c|c|c|c|c|c|c|c|}
\hline$p q$ & 0 & 1 & 2 & 3 & 4 & 5 & 6 & 7 & 8 \\
\hline 0 & $O(1)$ & $O(1)$ & $U(1)$ & $S p(1)$ & $S p(1)^{2}$ & $S p(2)$ & $U(4)$ & $O(8)$ & $O(8)^{2}$ \\
\hline 1 & $O(1)$ & $G L(1, \mathbb{R})$ & $S p(1, \mathbb{R})$ & $S p(1, \mathbb{C})$ & $S p(1,1)$ & $G L(2, \mathbb{H})$ & $O^{*}(8)$ & $O(8, \mathbb{C})$ & $O(8,8)$ \\
\hline 2 & $U(1)$ & $S p(1, \mathbb{R})$ & $S p(1, \mathbb{R})^{2}$ & $S p(2, \mathbb{R})$ & $U(2,2)$ & $O^{*}(8)$ & $O^{*}(8)^{2}$ & $O^{*}(16)$ & $U(8,8)$ \\
\hline 3 & $S p(1)$ & $S p(1, \mathbb{C})$ & $S p(2, \mathbb{R})$ & $G L(4, \mathbb{R})$ & $O(4,4)$ & $O(8, \mathbb{C})$ & $O^{*}(16)$ & $G L(8, \mathbb{H})$ & $S p(8,8)$ \\
\hline 4 & $S p(1)^{2}$ & $S p(1,1)$ & $U(2,2)$ & $O(4,4)$ & $O(4,4)^{2}$ & $O(8,8)$ & $U(8,8)$ & $S p(8,8)$ & $S p(8,8)^{2}$ \\
\hline 5 & $S p(2)$ & $G L(2, \mathbb{H})$ & $O^{*}(8)$ & $O(8, \mathbb{C})$ & $O(8,8)$ & $G L(16, \mathbb{R})$ & $S p(16, \mathbb{R})$ & $S p(16, \mathbb{C})$ & $S p(16,16)$ \\
\hline 6 & $U(4)$ & $O^{*}(8)$ & $O^{*}(8)^{2}$ & $O^{*}(16)$ & $U(8,8)$ & $S p(16, \mathbb{R})$ & $S p(16, \mathbb{R})^{2}$ & $S p(32, \mathbb{R})$ & $U(32,32)$ \\
\hline 7 & $O(8)$ & $O(8, \mathbb{C})$ & $O^{*}(16)$ & $G L(8, \mathbb{H})$ & $S p(8,8)$ & $S p(16, \mathbb{C})$ & $S p(32, \mathbb{R})$ & $G L(64, \mathbb{R})$ & $O(64,64)$ \\
\hline 8 & $O(8)^{2}$ & $O(8,8)$ & $U(8,8)$ & $S p(8,8)$ & $S p(8,8)^{2}$ & $S p(16,16)$ & $U(32,32)$ & $O(64,64)$ & $O(64,64)^{2}$ \\
\hline
\end{tabular}

Table 4.4.3

Remark 4.4.5. As one may observe from Table 4.4.3, the pair $(G(p+1, q), G(p, q))$ forms a symmetric pair, and likewise $(G(p, q+1), G(p, q))$. In fact, $\tau_{1}^{+}$is the involutive automorphism of the group $G(p+1, q)$ such that the group of the fixed points $G(p+1, q)^{\tau_{1}^{+}}=G(p, q)$, and likewise, $G(p, q+1)^{\tau_{1}^{-}}=G(p, q)$, where $\tau_{1}^{ \pm}$ will be defined in (4.5.1).

4.5. Definition and Lemmas. For later argument, it will be convenient to use the following notation for the basis $\left\{e_{j}\right\}$ of $E$ :

$$
v_{j}^{+}:=e_{j}(1 \leq j \leq p), \quad v_{j}^{-}:=e_{p+j}(1 \leq j \leq q) .
$$

Furthermore, we write $\tau_{i}^{ \pm}$for the involutive automorphism induced from reflection with respect to the normal direction $v_{i}^{ \pm}$, namely,

$$
\tau_{i}^{ \pm}:=\tau_{v_{i}^{ \pm}} .
$$

Note that $\tau_{i}^{ \pm}$mutually commute because $e_{i}^{ \pm}$are orthogonal to each other.

For pairs of natural numbers $\mathcal{K}=\left(k_{+}, k_{-}\right), \mathcal{L}=\left(l_{+}, l_{-}\right)$, we define:

$$
\begin{aligned}
\Delta \mathcal{K} & :=k_{+}-k_{-}, \\
\mathcal{K}^{\vee} & :=\left(k_{-}, k_{+}\right), \\
\mathcal{K} \pm \mathcal{L} & :=\left(k_{+} \pm l_{+}, k_{-} \pm l_{-}\right) .
\end{aligned}
$$


Definition 4.5.1. Let $\mathcal{M}=(p, q), \mathcal{K}=\left(k_{+}, k_{-}\right)$and $\mathcal{L}=\left(l_{+}, l_{-}\right)$be pairs of natural numbers such that $\mathcal{M}=\mathcal{K}+\mathcal{L}$. We define elements $V_{\mathcal{K}}, J_{ \pm}, J$ in $C(p, q)$, and involutive automorphisms $T_{\mathcal{L}}$ of $C(p, q)$ by:

$$
\begin{aligned}
V_{\mathcal{K}} & :=v_{1}^{+} \cdots v_{k_{+}}^{+} v_{1}^{-} \cdots v_{k_{-}}^{-}, \\
J_{+} & :=V_{p, 0}, \\
J_{-} & :=V_{0, q}, \\
J & :=V_{p, q}=J_{+} J_{-}, \\
T_{\mathcal{L}} & :=\tau_{p-l_{+}+1}^{+} \cdots \tau_{p}^{+} \tau_{q-l_{-}+1}^{-} \cdots \tau_{q}^{-} .
\end{aligned}
$$

We also use the notation $m_{+}:=p$ and $m_{-}:=q$ so that $\mathcal{M}=\left(m_{+}, m_{-}\right)$. In particular, $\Delta \mathcal{M}=p-q$. Then, we have from definition:

$$
T_{\mathcal{L}}\left(v_{i}^{ \pm}\right)= \begin{cases}v_{i}^{ \pm} & \text {if } 1 \leq i \leq m_{ \pm}-l_{ \pm} \\ -v_{i}^{ \pm} & \text {if } m_{ \pm}-l_{ \pm}<i \leq m_{ \pm} .\end{cases}
$$

A straightforward computation shows

$$
V_{\mathcal{K}}^{2}= \begin{cases}1 & \text { if } \Delta \mathcal{K} \equiv 0,1 \quad \bmod 4 \\ -1 & \text { if } \Delta \mathcal{K} \equiv 2,3 \bmod 4\end{cases}
$$

In particular, we have:

$$
J^{2}= \begin{cases}1 & \text { if } \Delta \mathcal{M} \equiv 0,1 \quad \bmod 4, \\ -1 & \text { if } \Delta \mathcal{M} \equiv 2,3 \quad \bmod 4 .\end{cases}
$$

Remark 4.5.2. We have $J a=a J$ for any $a \in C(p, q)$ if $\Delta \mathcal{M} \equiv 1 \bmod 2$. It then follows from (4.5.4) that $J$ becomes a central element satisfying $J^{2}=-1$ if and only if $\Delta \mathcal{M} \equiv 3 \bmod 4$. In this case, $C(p, q)$ is endowed with the $\mathbb{C}$ algebra structure with complex structure $J$. We recall Proposition 4.4 .1 asserts particularly that $C(p, q)$ is isomorphic to $M(n, \mathbb{C})$ if $m_{+}-m_{-}-1 \equiv \pm 2 \bmod 8$, namely, if $\Delta \mathcal{M} \equiv 3 \bmod 4$.

Next, given an automorphism $\tau$ of $C(p, q)$, we denote by $C(p, q)^{\tau}$ the subalgebra consisting of $\tau$-fixed elements:

$$
C(p, q)^{\tau}:=\{a \in C(p, q): \tau(a)=a\} .
$$

Example 4.5.3. Retain the notation as in (4.5.1) and Definition 4.5.1.

1) $C(p, q)^{T_{p, q}}=C_{\text {even }}(p, q)$.

2) $C(p, q)^{\tau_{i}^{+}} \simeq C(p-1, q) \quad(1 \leq i \leq p)$.

3) $C(p, q)^{\tau_{i}^{-}} \simeq C(p, q-1) \quad(1 \leq i \leq q)$. 
The composition of $a \mapsto^{t} a$ (see (4.3.3)) and $T_{0, q}$ gives rise to an anti-automor -phism, denoted by $a \mapsto a^{*}$, of the $\mathbb{R}$-algebra $C(p, q)$ :

$$
a^{*}:=T_{0, q}\left({ }^{t} a\right) \quad(a \in C(p, q)) .
$$

We shall see in Lemma 4.5.5 that

$$
a^{*}=T_{p, 0}\left({ }^{t} a\right) \quad\left(a \in C_{\text {even }}(p, q)\right) .
$$

All of the isomorphisms, say $\phi$, that we shall need later satisfy $\phi\left(a^{*}\right)=\phi(a)^{*}$ (see Lemma 4.7.10), but $\phi\left({ }^{t} a\right)$ is not always equal to ${ }^{t} \phi(a)$. Therefore, in order to find the structure of the group $G(p, q)$, it is more convenient to rewrite the definition of $G(p, q)$ (see Definition 4.3.1) by means of $a^{*}$ instead of ${ }^{t} a$. For this purpose, we prepare the following definition and lemma:

Definition 4.5.4. Retain the notation as in Definition 4.5.1 and (4.5.5). We define the groups $\operatorname{Aut}\left(J_{+}\right)$and $\operatorname{Aut}\left(J_{-}\right)$as

$$
\operatorname{Aut}\left(J_{ \pm}\right):=\left\{a \in C(p, q): a^{*} J_{ \pm} a=J_{ \pm}\right\}
$$

Then, we have:

Lemma 4.5.5. 1) $J_{+}^{-1} a J_{+}=J_{-}^{-1} a J_{-}=T_{p, 0}(a)=T_{0, q}(a)$ for any $a \in C_{\text {even }}(p, q)$.

2) $G(p, q)=C_{\text {even }}(p, q) \cap \operatorname{Aut}\left(J_{+}\right)=C_{\text {even }}(p, q) \cap \operatorname{Aut}\left(J_{-}\right)$.

Proof. 1) By the definition of $J_{+}$and $J_{-}$, we have:

$$
\begin{array}{ll}
J_{+}^{-1} v_{i}^{+} J_{+}=(-1)^{p-1} v_{i}^{+}, & J_{+}^{-1} v_{i}^{-} J_{+}=(-1)^{p} v_{i}^{-}, \\
J_{-}^{-1} v_{i}^{+} J_{-}=(-1)^{q} v_{i}^{+}, & J_{-}^{-1} v_{i}^{-} J_{-}=(-1)^{q-1} v_{i}^{-} .
\end{array}
$$

In light of (4.5.2), we obtain the following equation for any $a \in C(p, q)$ :

$$
J_{+}^{-1} a J_{+}=\left\{\begin{array}{ll}
T_{p, 0}(a) & \text { if } p \text { is even, } \\
T_{0, q}(a) & \text { if } p \text { is odd, }
\end{array} \quad J_{-}^{-1} a J_{-}= \begin{cases}T_{0, q}(a) & \text { if } q \text { is even, } \\
T_{p, 0}(a) & \text { if } q \text { is odd. }\end{cases}\right.
$$

On the other hand, because $T_{p, q}(a)=a$ for any $a \in C_{\text {even }}(p, q)$ by Example 4.5.3(1), we obtain:

$$
T_{p, 0}(a)=T_{p, 0} \circ T_{p, q}(a)=T_{0, q}(a) .
$$

Thus, the statement (1) has been proved.

2) Note that $a^{*} \in C_{\text {even }}(p, q)$ if $a \in C_{\text {even }}(p, q)$. Then we have:

$$
J_{+}^{-1} a^{*} J_{+}=J_{-}^{-1} a^{*} J_{-}=T_{0, q}\left(a^{*}\right)={ }^{t} a .
$$

Here, the last equality follows from (4.5.5). Thus, (2) follows. 
Remark 4.5.6. If $p-q \equiv 1 \bmod 2$, then the above proof shows that $J_{+}^{-1} a J_{+}=$ $J_{-}^{-1} a J_{-}$. Therefore, we have $C(p, q) \cap \operatorname{Aut}\left(J_{+}\right)=C(p, q) \cap \operatorname{Aut}\left(J_{-}\right)$.

4.6. Proof of Proposition 4.4.1. This subsection establishes various isomorphisms among Clifford algebras (Lemma

4.6.2) and completes the proof of the structural result (Lemma 4.4.3 and Proposition 4.4.1) of the Clifford algebra $C(p, q)$. First, for $(p, q)$ such that $p+q \leq 2$, Proposition 4.4.1 is directly verified by the following lemma:

Lemma 4.6.1. We have the following isomorphisms as $\mathbb{R}$-algebras:

$$
\begin{aligned}
& \psi_{0,1}: C(0,1) \stackrel{\sim}{\rightarrow} \mathbb{C}, \\
& \psi_{1,0}: C(1,0) \stackrel{\sim}{\rightarrow} \mathbb{R} \oplus \mathbb{R}, \\
& \psi_{0,2}: C(0,2) \stackrel{\sim}{\rightarrow} \mathbb{H}, \\
& \psi_{1,1}: C(1,1) \stackrel{\sim}{\rightarrow} M(2, \mathbb{R}), \\
& \psi_{2,0}: C(2,0) \stackrel{\sim}{\rightarrow} M(2, \mathbb{R}) .
\end{aligned}
$$

Proof. The linear map $\psi_{1,1}: \mathbb{R}^{1,1} \rightarrow M(2, \mathbb{R})$ given by

$$
v_{1}^{+} \mapsto\left(\begin{array}{cc}
1 & \\
& -1
\end{array}\right), \quad v_{1}^{-} \mapsto\left(\begin{array}{c}
-1 \\
1
\end{array}\right)
$$

induces the algebra isomorphism $\psi_{1,1}: C(1,1) \stackrel{\sim}{\rightarrow} M(2, \mathbb{R})$ by the universality of the $\mathbb{R}$-algebra $C(1,1)$. Other isomorphisms are well-known, but we write down these maps explicitly on the generators for later purposes.

$$
\begin{array}{llll}
\psi_{0,1}: C(0,1) \stackrel{\sim}{\rightarrow} \mathbb{C}, & v_{1}^{-} \mapsto i, & & \\
\psi_{1,0}: C(1,0) \stackrel{\sim}{\rightarrow} \mathbb{R} \oplus \mathbb{R}, & v_{1}^{+} \mapsto(1,-1), & \\
\psi_{0,2}: C(0,2) \stackrel{\sim}{\rightarrow} \mathbb{H}, & v_{1}^{-} \mapsto i, & v_{2}^{-} \mapsto j, \\
\psi_{2,0}: C(2,0) \stackrel{\sim}{\rightarrow} M(2, \mathbb{R}), & v_{1}^{+} \mapsto\left(\begin{array}{c}
1 \\
-1
\end{array}\right), & v_{2}^{+} \mapsto\left(\begin{array}{c}
1 \\
1
\end{array}\right) .
\end{array}
$$

For general $(p, q)$ such that $p+q \geq 3$, one shall find the structure of $C(p, q)$ inductively based on the following:

Proof of Proposition 4.4.1. (4.4.2) reduces the proof of Proposition 4.4.1 to the case $|p-q-1| \leq 4$. Then (4.4.1) with $r=1$ reduces it to the case $-4 \leq p-q-1 \leq$ 0 . 
Case 1) $p-q-1=0$.

An iterative use of $\phi_{1,1}$ yields:

$$
C(p, q)=C(q+1, q) \simeq\left(\bigotimes^{q} C(1,1)\right) \otimes C(1,0) \simeq M\left(2^{q}, \mathbb{R}\right) \otimes(\mathbb{R} \oplus \mathbb{R}) .
$$

Here, the last isomorphism is induced by $\psi_{1,1}: C(1,1) \stackrel{\sim}{\rightarrow} M(2, \mathbb{R})$ and $\psi_{1,0}$ : $C(1,0) \stackrel{\sim}{\rightarrow} \mathbb{R} \oplus \mathbb{R}$.

Case 2) $p-q-1=-1,-2,-3$, or -4 .

We write $q=p+s(s=0,1,2$, or 3$)$. Then we have

$$
C(p, q)=C(p, p+s) \simeq\left(\bigotimes^{p} C(1,1)\right) \otimes C(0, s) \simeq M\left(2^{p}, \mathbb{R}\right) \otimes C(0, s) .
$$

Thus, Proposition 4.4.1 is reduced to the isomorphism $C(0,0) \simeq \mathbb{R}, C(0,1) \simeq \mathbb{C}$, $C(0,2) \simeq \mathbb{H}$ (see Lemma 4.6.1) or $C(0,3) \simeq \mathbb{H} \oplus \mathbb{H}$, where the last isomorphism is derived from the composition:

$$
C(0,3) \underset{\phi_{0,2}}{\stackrel{\sim}{\longrightarrow}} C(0,2) \otimes C(1,0) \underset{\psi_{0,2} \otimes \psi_{1,0}}{\stackrel{\sim}{\longrightarrow}} \mathbb{H} \otimes(\mathbb{R} \oplus \mathbb{R}) \simeq \mathbb{H} \oplus \mathbb{H} .
$$

Hence, we have proved Proposition 4.4.1.

Lemma 4.6.2. For $\mathcal{M}=\left(m_{+}, m_{-}\right) \in \mathbb{N}^{2}$, we write simply $C(\mathcal{M})$ for the Clifford algebra $C\left(m_{+}, m_{-}\right)$.

1) Suppose $\mathcal{K}, \mathcal{L} \in \mathbb{N}^{2}$ such that $\mathcal{M}=\mathcal{K}+\mathcal{L}$. Then, we have the following $\mathbb{R}$-algebra isomorphism $\phi_{\mathcal{K}}$ as an extension of the linear map below:

$$
\phi_{\mathcal{K}}: C(\mathcal{K}+\mathcal{L}) \rightarrow\left\{\begin{array}{lll}
C(\mathcal{K}) \otimes C(\mathcal{L}) & \text { if } \Delta \mathcal{K} \equiv 0 & \bmod 4, \\
C\left(\mathcal{K}+\mathcal{L}^{\vee}\right) & \text { if } \Delta \mathcal{K} \equiv 1 \bmod 4, \\
C(\mathcal{K}) \otimes C\left(\mathcal{L}^{\vee}\right) & \text { if } \Delta \mathcal{K} \equiv 2 \bmod 4, \\
C(\mathcal{K}+\mathcal{L}) & \text { if } \Delta \mathcal{K} \equiv 3 \bmod 4 .
\end{array}\right.
$$

Case i) $\Delta \mathcal{K} \equiv 0 \bmod 4 . \phi_{\mathcal{K}}: E \rightarrow C(\mathcal{K}) \otimes C(\mathcal{L})$ is given by

$$
v_{i}^{ \pm} \mapsto v_{i}^{ \pm} \otimes 1 \quad\left(1 \leq i \leq k_{ \pm}\right), \quad v_{k_{ \pm}+i}^{ \pm} \mapsto V_{\mathcal{K}} \otimes v_{i}^{ \pm} \quad\left(1 \leq i \leq l_{ \pm}\right) .
$$

Case ii) $\Delta \mathcal{K} \equiv 1 \bmod 4 . \phi_{\mathcal{K}}: E \rightarrow C\left(\mathcal{K}+\mathcal{L}^{\vee}\right)$ is given by

$$
v_{i}^{ \pm} \mapsto v_{i}^{ \pm} \quad\left(1 \leq i \leq k_{ \pm}\right), \quad v_{k_{ \pm}+i}^{ \pm} \mapsto V_{\mathcal{K}} v_{k_{\mp}+i}^{\mp} \quad\left(1 \leq i \leq l_{ \pm}\right) .
$$

Case iii) $\Delta \mathcal{K} \equiv 2 \bmod 4 . \phi_{\mathcal{K}}: E \rightarrow C(\mathcal{K}) \otimes C\left(\mathcal{L}^{\vee}\right)$ is given by

$$
v_{i}^{ \pm} \mapsto v_{i}^{ \pm} \otimes 1 \quad\left(1 \leq i \leq k_{ \pm}\right), \quad v_{k_{ \pm}+i}^{ \pm} \mapsto V_{\mathcal{K}} \otimes v_{i}^{\mp} \quad\left(1 \leq i \leq l_{ \pm}\right) .
$$

Case iv) $\Delta \mathcal{K} \equiv 3 \bmod 4 . \phi_{\mathcal{K}}: E \rightarrow C(\mathcal{K}+\mathcal{L})$ is given by

$$
v_{i}^{ \pm} \mapsto v_{i}^{ \pm} \quad\left(1 \leq i \leq k_{ \pm}\right), \quad v_{k_{ \pm}+i}^{ \pm} \mapsto V_{\mathcal{K}} v_{k_{ \pm}+i}^{ \pm} \quad\left(1 \leq i \leq l_{ \pm}\right) .
$$


2) Furthermore, $\phi_{\mathcal{K}}$ transfers the automorphism $\tau_{i}^{ \pm}$of the Clifford algebra $C(p, q)$ to the following automorphism:

(i) $\tau_{i}^{ \pm} \mapsto \tau_{i}^{ \pm} \otimes T_{\mathcal{L}} \quad\left(1 \leq i \leq k_{ \pm}\right), \quad \tau_{k_{ \pm}+i}^{ \pm} \mapsto \mathrm{id} \otimes \tau_{i}^{ \pm} \quad\left(1 \leq i \leq l_{ \pm}\right)$,

(ii) $\tau_{i}^{ \pm} \mapsto \tau_{i}^{ \pm} T_{\mathcal{L}^{\vee}} \quad\left(1 \leq i \leq k_{ \pm}\right), \quad \tau_{k_{ \pm}+i}^{ \pm} \mapsto \tau_{\mp+i}^{\mp} \quad\left(1 \leq i \leq l_{ \pm}\right)$,

(iii) $\tau_{i}^{ \pm} \mapsto \tau_{i}^{ \pm} \otimes T_{\mathcal{L}^{\vee}} \quad\left(1 \leq i \leq k_{ \pm}\right), \quad \tau_{k_{ \pm}+i}^{ \pm} \mapsto \mathrm{id} \otimes \tau_{i}^{\mp} \quad\left(1 \leq i \leq l_{ \pm}\right)$,

(iv) $\tau_{i}^{ \pm} \mapsto \tau_{i}^{ \pm} T_{\mathcal{L}} \quad\left(1 \leq i \leq k_{ \pm}\right), \quad \tau_{k_{ \pm}+i}^{ \pm} \mapsto \tau_{ \pm+i}^{ \pm} \quad\left(1 \leq i \leq l_{ \pm}\right)$.

Proof. 1) Let us give a proof in Case (i). Other cases are similar. To see $\phi_{\mathcal{K}}$ extends to an $\mathbb{R}$-algebra homomorphism, it is enough to verify the condition (4.3.2). This is so because $V_{\mathcal{K}}^{2}=1$ by (4.5.3) and $V_{\mathcal{K}} v_{i}^{ \pm}=-v_{i}^{ \pm} V_{\mathcal{K}}\left(1 \leq i \leq k_{ \pm}\right)$. Thus, the linear map $\phi_{\mathcal{K}}: E \rightarrow C(p, q)$ extends to an $\mathbb{R}$-algebra homomorphism $C(p, q) \rightarrow C(p, q)$.

Next, to see $\phi_{\mathcal{K}}$ is surjective, it is sufficient to check that the image of $\phi_{\mathcal{K}}$ contains generators of the algebra $C(\mathcal{K}) \otimes C(\mathcal{L})$. This is so because

$$
\phi_{\mathcal{K}}\left(v_{i}^{ \pm}\right)=v_{i}^{ \pm} \otimes 1\left(1 \leq i \leq k_{ \pm}\right), \quad \phi_{\mathcal{K}}\left(V_{\mathcal{K}} v_{k_{ \pm}+i}^{ \pm}\right)=1 \otimes v_{i}^{ \pm}\left(1 \leq i \leq l_{ \pm}\right)
$$

Finally, the injectivity of $\phi_{\mathcal{K}}$ follows from

$$
\operatorname{dim} C(p, q)=2^{p+q}=2^{k_{+}+k_{-}} 2^{l_{+}+l_{-}}=\operatorname{dim} C(\mathcal{K}) \otimes C(\mathcal{L}) .
$$

Thus, the statement (1) is proved.

2) Let us verify that $\phi_{\mathcal{K}}$ transfers $\tau_{1}^{+}$to $\tau_{1}^{+} \otimes T_{\mathcal{L}}$, or equivalently, $\phi_{\mathcal{K}} \circ \tau_{1}^{+}=$ $\left(\tau_{1}^{+} \otimes T_{\mathcal{L}}\right) \circ \phi_{\mathcal{K}}$ in Case (i) $\left(k_{+} \geq 1\right)$. Other cases are similar. This equation can be checked by the evaluation at the generators $\left(v_{1}^{+}, \ldots, v_{p}^{+}, v_{1}^{-}, \ldots, v_{q}^{-}\right)$as follows:

$$
\begin{aligned}
& \phi_{\mathcal{K}} \circ \tau_{1}^{+}\left(v_{1}^{+}\right)=-v_{1}^{+} \quad=\left(\tau_{1}^{+} \otimes T_{\mathcal{L}}\right) \circ \phi_{\mathcal{K}}\left(v_{1}^{+}\right), \\
& \phi_{\mathcal{K}} \circ \tau_{1}^{+}\left(v_{i}^{+}\right)=v_{i}^{+} \quad=\left(\tau_{1}^{+} \otimes T_{\mathcal{L}}\right) \circ \phi_{\mathcal{K}}\left(v_{i}^{+}\right) \quad\left(2 \leq i \leq k_{+}\right) \text {, } \\
& \phi_{\mathcal{K}} \circ \tau_{1}^{+}\left(v_{i}^{-}\right)=v_{i}^{-} \quad=\left(\tau_{1}^{+} \otimes T_{\mathcal{L}}\right) \circ \phi_{\mathcal{K}}\left(v_{i}^{-}\right) \quad\left(1 \leq i \leq k_{-}\right), \\
& \phi_{\mathcal{K}} \circ \tau_{1}^{+}\left(v_{k_{ \pm}+i}^{ \pm}\right)=V_{\mathcal{K}} \otimes v_{i}^{ \pm} \quad=\left(\tau_{1}^{+} \otimes T_{\mathcal{L}}\right) \circ \phi_{\mathcal{K}}\left(v_{k_{ \pm}+i}^{ \pm}\right) \quad\left(1 \leq i \leq k_{ \pm}\right) .
\end{aligned}
$$


We pin down $\phi_{\mathcal{K}}$ explicitly in the cases $\mathcal{K}=(0,1),(1,0),(0,2),(1,1),(2,0)$ and $\mathcal{L}=(p, q)$.

$$
\begin{aligned}
& \phi_{0,1}: \quad C(p, q+1) \quad \stackrel{\sim}{\rightarrow} C(p, q+1) \\
& v_{i}^{+} \quad \mapsto \quad v_{1}^{-} v_{i}^{+} \quad(1 \leq i \leq p) \\
& \begin{array}{cccc}
v_{1}^{-} & \mapsto & v_{1}^{-} & \\
v_{i+1}^{-} & \mapsto & v_{1}^{-} v_{i}^{-} & (1 \leq i \leq q),
\end{array} \\
& \phi_{1,0}: C(p+1, q) \stackrel{\sim}{\rightarrow} C(q+1, p) \\
& \begin{array}{ccccc}
v_{1}^{+} & \mapsto & v_{1}^{+} & \\
v_{i+1}^{+} & \mapsto & v_{1}^{+} v_{i}^{-} & & (1 \leq i \leq p) \\
v_{i}^{-} & & v_{1}^{+} v_{i+1}^{+} & (1 \leq i \leq q),
\end{array} \\
& \phi_{0,2}: \quad C(p, q+2) \stackrel{\sim}{\rightarrow} C(0,2) \otimes C(q, p) \\
& v_{i}^{+} \quad \mapsto \quad v_{1}^{-} v_{2}^{-} \otimes v_{i}^{-} \quad(1 \leq i \leq p) \\
& v_{i}^{-} \quad \mapsto \quad v_{i}^{-} \otimes 1 \quad(i=1,2) \\
& v_{i+2}^{-} \quad \mapsto \quad v_{1}^{-} v_{2}^{-} \otimes v_{i}^{+} \quad(1 \leq i \leq q), \\
& \phi_{1,1}: C(p+1, q+1) \stackrel{\sim}{\rightarrow} C(1,1) \otimes C(p, q) \\
& v_{1}^{ \pm} \quad \mapsto \quad v_{1}^{ \pm} \otimes 1 \\
& v_{i+1}^{+} \quad \mapsto \quad v_{1}^{+} v_{1}^{-} \otimes v_{i}^{+} \quad(1 \leq i \leq p) \\
& v_{i+1}^{-} \quad \mapsto \quad v_{1}^{+} v_{1}^{-} \otimes v_{i}^{-} \quad(1 \leq i \leq q), \\
& \phi_{2,0}: \quad C(p+2, q) \stackrel{\sim}{\rightarrow} C(2,0) \otimes C(q, p)
\end{aligned}
$$

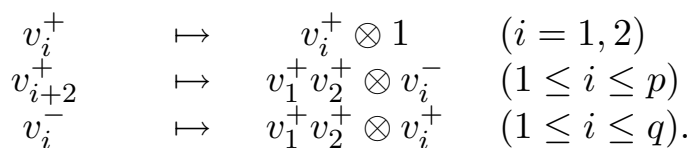

Remark 4.6.3. Among the above isomorphisms, positive (or negative) definite cases such as the isomorphisms $\phi_{0,2}: C(0, q+2) \stackrel{\sim}{\rightarrow} C(0,2) \otimes C(q, 0)$ and $\phi_{2,0}:$ $C(p+2,0) \stackrel{\sim}{\rightarrow} C(2,0) \otimes C(0, p)$ are found in [ABS64].

4.7. Isomorphism $\lambda$. In this subsection, we introduce an $\mathbb{R}$-algebra isomorphism $\lambda_{\mathcal{K}}$ (see Lemma 4.7.2). The effect of $\lambda_{\mathcal{K}}$, as we shall show in Lemmas 4.7.7 and 4.7.9, is to make the 'degree' of $J_{ \pm}, T_{p, 0}$ or $T_{0, q}$ down to one. These two lemmas will play a key role in finding the group structure of $G(p, q)$ in Section 4.8.

Suppose that $a \in C(p, q)$ is of the form

$$
a=a_{1} \cdots a_{s},
$$


where $a_{1}, \ldots, a_{s}$ are distinct elements among the set of the generators $v_{1}^{+}, \cdots, v_{p}^{+}$, $v_{1}^{-}, \cdots, v_{q}^{-}$. Then

$$
\left\{a_{1}, \ldots, a_{s}\right\}=\left\{v_{i_{1}}^{+}, \ldots, v_{i_{t}}^{+}, v_{j_{1}}^{-}, \ldots, v_{j_{r}}^{-}\right\} \quad(s=t+r)
$$

is independent of the expression $a=a_{1} \cdots a_{s}$. We set

$$
\begin{aligned}
\operatorname{index}(a) & :=t-r, \\
\operatorname{deg}(a) & :=s \quad(=t+r) .
\end{aligned}
$$

Suppose another element $b \in C(p, q)$ is also expressed as a product $b=b_{1} \cdots b_{s^{\prime}}$ of the generators. We denote by $\operatorname{deg}(a, b)$ the cardinality of the generators occurred in both $a$ and $b$. For example:

$$
\begin{aligned}
\operatorname{index}\left(v_{1}^{+} v_{2}^{-} v_{2}^{+}\right) & =\#\left\{v_{1}^{+}, v_{2}^{+}\right\}-\#\left\{v_{2}^{-}\right\}=1, \\
\operatorname{deg}\left(v_{1}^{+} v_{2}^{-} v_{2}^{+}\right) & =\#\left\{v_{1}^{+}, v_{2}^{-}, v_{2}^{+}\right\}=3, \\
\operatorname{deg}\left(v_{1}^{+} v_{2}^{-} v_{2}^{+}, v_{1}^{-} v_{2}^{+} v_{1}^{+}\right) & =\#\left\{v_{1}^{+}, v_{2}^{+}\right\}=2 .
\end{aligned}
$$

With this notation, we have:

Lemma 4.7.1. For $a$ and $b \in C(p, q)$ of the form (4.7.1), we have:

1) $a^{2}=(-1)^{\frac{1}{2} \operatorname{index}(a)(\operatorname{index}(a)-1)}= \begin{cases}1 & \text { if } \operatorname{index}(a) \equiv 0,1 \bmod 4, \\ -1 & \text { if } \operatorname{index}(a) \equiv 2,3 \bmod 4 .\end{cases}$

2) $a b=(-1)^{\operatorname{deg}(a) \cdot \operatorname{deg}(b)-\operatorname{deg}(a, b)} b a$.

Proof. Follows from straightforward computation.

Lemma 4.7.2. Let $\mathcal{M}=\left(m_{+}, m_{-}\right):=(p, q)$ and $\mathcal{K}=\left(k_{+}, k_{-}\right)$satisfy $1 \leq$ $k_{ \pm} \leq m_{ \pm}$. We set $V:=V_{k_{+}-1, k_{-}-1}\left(=v_{1}^{+} \cdots v_{k_{+}-1}^{+} v_{1}^{-} \cdots v_{k_{-}-1}^{-}\right)$. Then the linear map $\lambda_{\mathcal{K}}: E \rightarrow C(p, q)$ given below extends to an $\mathbb{R}$-algebra automorphism $\lambda_{\mathcal{K}}: C(p, q) \stackrel{\sim}{\rightarrow} C(p, q)$.

Case 1) $\Delta \mathcal{K} \equiv 0 \bmod 2$.

$$
\begin{aligned}
& v_{i}^{ \pm} \mapsto v_{k_{+}}^{+} v_{k_{-}}^{-} v_{i}^{ \pm} \\
& \left(1 \leq i \leq k_{ \pm}-1\right), \\
& v_{k_{ \pm}}^{ \pm} \mapsto\left\{\begin{array}{lll}
V v_{k_{ \pm}}^{ \pm} & \text {if } \Delta \mathcal{K} \equiv 0 \quad \bmod 4, \\
V v_{k_{\mp}}^{\mp} & \text { if } \Delta \mathcal{K} \equiv 2 \quad \bmod 4,
\end{array}\right. \\
& v_{i}^{ \pm} \mapsto v_{i}^{ \pm} \\
& \left(k_{ \pm}+1 \leq i \leq m_{ \pm}\right) .
\end{aligned}
$$


Case 2) $\Delta \mathcal{K} \equiv 1 \bmod 2$.

$$
\begin{aligned}
& v_{i}^{ \pm} \mapsto v_{i}^{ \pm} \\
& v_{k_{ \pm}}^{ \pm} \mapsto\left\{\begin{array}{lll}
V v_{k_{\mp}}^{\mp} & \text { if } \Delta \mathcal{K} \equiv 1 \quad \bmod 4, \\
V v_{k_{ \pm}}^{ \pm} & \text {if } \Delta \mathcal{K} \equiv 3 \quad \bmod 4,
\end{array}\right. \\
& v_{i}^{ \pm} \mapsto v_{k_{+}}^{+} v_{k_{-}}^{-} v_{i}^{ \pm} \quad\left(k_{ \pm}+1 \leq i \leq m_{ \pm}\right) .
\end{aligned}
$$

Proof. It is easy to verify the condition (4.3.2). Then, $\lambda_{\mathcal{K}}$ extends to an $\mathbb{R}$ algebra automorphism $C(p, q) \rightarrow C(p, q)$ by the universality. Furthermore, a simple computation on the generators shows:

$$
\left(\lambda_{\mathcal{K}}\right)^{2}= \begin{cases}\text { id } & \text { if } \Delta \mathcal{K} \equiv 0,1 \quad \bmod 4 \\ \tau_{k_{+}}^{+} \tau_{k_{-}}^{-} & \text {if } \Delta \mathcal{K} \equiv 2,3 \quad \bmod 4\end{cases}
$$

Hence, $\lambda_{\mathcal{K}}$ is bijective.

Lemma 4.7.3. Retain the setting as in Lemma 4.7.2. Then, the $\mathbb{R}$-algebra isomorphism $\lambda_{\mathcal{K}}$ transfers the automorphism $\tau_{i}^{ \pm}$as follows:

Case 1) $\Delta \mathcal{K} \equiv 0 \bmod 2$.

$$
\begin{aligned}
& \tau_{i}^{ \pm} \mapsto \tau_{i}^{ \pm} \quad\left(1 \leq i \leq k_{ \pm}-1\right), \\
& \tau_{k_{ \pm}}^{ \pm} \mapsto \begin{cases}\tau_{k_{ \pm}}^{ \pm} T_{\mathcal{M}} T_{\mathcal{M}-\mathcal{K}} & \text { if } \Delta \mathcal{K} \equiv 0 \quad \bmod 4 \\
\tau_{k_{\mp}}^{\mp} T_{\mathcal{M}} T_{\mathcal{M}-\mathcal{K}} & \text { if } \Delta \mathcal{K} \equiv 2 \quad \bmod 4\end{cases} \\
& \tau_{i}^{ \pm} \mapsto \tau_{i}^{ \pm} \tau_{k_{+}}^{+} \tau_{k_{-}}^{-} \quad\left(k_{ \pm}+1 \leq i \leq m_{ \pm}\right) .
\end{aligned}
$$

Case 2) $\Delta \mathcal{K} \equiv 1 \bmod 2$.

$$
\begin{array}{rlrl}
\tau_{i}^{ \pm} \mapsto \tau_{i}^{ \pm} \tau_{k_{+}}^{+} \tau_{k_{-}}^{-} & & \left(1 \leq i \leq k_{ \pm}-1\right), \\
\tau_{k_{ \pm}}^{ \pm} & \mapsto\left\{\begin{array}{lll}
\tau_{k_{ \pm}}^{ \pm} T_{\mathcal{M}-\mathcal{K}} & \text { if } \Delta \mathcal{K} \equiv 1 & \bmod 4, \\
\tau_{k_{\mp}}^{\mp} T_{\mathcal{M}-\mathcal{K}} & \text { if } \Delta \mathcal{K} \equiv 3 & \bmod 4,
\end{array}\right. \\
\tau_{i}^{ \pm} \mapsto \tau_{i}^{ \pm} & \quad\left(k_{ \pm}+1 \leq i \leq m_{ \pm}\right) .
\end{array}
$$

Proof. The proof goes similarly as in Lemma 4.6.2(2).

Remark 4.7.4. The isomorphism $\lambda_{\mathcal{K}}$ is essentially the composition $\phi_{1,1}^{-1} \circ \phi_{\mathcal{K}^{\prime}}^{-1} \circ$ $\phi_{1,1} \circ \phi_{\mathcal{K}^{\prime}}$ up to an automorphism induced by switching of generators, where $\mathcal{K}^{\prime}=\left(k_{+}-1, k_{-}-1\right)$.

For example, in the case $\Delta \mathcal{K} \equiv 0 \bmod 4, \lambda_{\mathcal{K}}$ may be defined as the composition of the following $\mathbb{R}$-algebra isomorphisms:

$$
\begin{aligned}
& C(p, q)=C\left(\mathcal{K}^{\prime}+\mathcal{L}^{\prime}\right) \simeq C\left(\mathcal{K}^{\prime}\right) \otimes C\left(\mathcal{L}^{\prime}\right) \simeq C\left(\mathcal{K}^{\prime}\right) \otimes C(1,1) \otimes C\left(l_{+}^{\prime}-1, l_{-}^{\prime}-1\right) \\
& \simeq C(1,1) \otimes C\left(\mathcal{K}^{\prime}\right) \otimes C\left(l_{+}^{\prime}-1, l_{-}^{\prime}-1\right) \simeq C(1,1) \otimes C(p-1, q-1) \simeq C(p, q) .
\end{aligned}
$$


In Lemma 4.7.2, we have adopted an alternative and direct definition of $\lambda_{\mathcal{K}}$.

As we shall see in the following lemma, the point of introducing the isomorphism $\lambda_{\mathcal{K}}$ is that it sends certain elements of "higher degree" such as $V_{*}$ or $T_{*}$ into those of degree one.

Lemma 4.7.5. Suppose that the pairs $\mathcal{M}=\left(m_{+}, m_{-}\right)$and $\mathcal{K}=\left(k_{+}, k_{-}\right)$satisfy $1 \leq k_{ \pm} \leq m_{ \pm}$. Then we have:

1) $\lambda_{\mathcal{K}}\left(V_{k_{+}-1, k_{-}-1} v_{k_{ \pm}}^{ \pm}\right)= \begin{cases}\varepsilon v_{k_{ \pm}}^{ \pm} & \text {if } \Delta \mathcal{K} \equiv 0,3 \bmod 4 \\ \varepsilon v_{k_{\mp}}^{\mp} & \text { if } \Delta \mathcal{K} \equiv 1,2 \bmod 4 .\end{cases}$

2) $\lambda_{\mathcal{K}}\left(\tau_{k_{ \pm}}^{ \pm} T_{\mathcal{M}-\mathcal{K}}\right)= \begin{cases}\tau_{k_{\mp}}^{\mp} & \text { if } \Delta \mathcal{K} \equiv 1 \bmod 4 \\ \tau_{k_{ \pm}}^{ \pm} & \text {if } \Delta \mathcal{K} \equiv 3 \bmod 4\end{cases}$

Here, $\varepsilon \in\{1,-1\}$.

Proof. 1) By a simple computation, one finds $\lambda_{\mathcal{K}}\left(V_{k_{+}-1, k_{-}-1}\right)=V_{k_{+}-1, k_{-}-1}$. Then, the statement (1) follows readily from the definition of $\lambda_{\mathcal{K}}\left(v_{k_{ \pm}}^{ \pm}\right)$(see Lemma 4.7.2).

2) Similarly, the statement (2) is obtained by combining the formula $\lambda_{\mathcal{K}}\left(T_{\mathcal{M}-\mathcal{K}}\right)=$ $T_{\mathcal{M}-\mathcal{K}}$ if $\Delta \mathcal{K} \equiv 1 \bmod 2$ and Lemma 4.7.3(1).

Remark 4.7.6. It is not hard to specify $\varepsilon= \pm 1$ in Lemma 4.7.5 (1), but we do not need this information in this article.

We have already seen in Lemma 4.6 .2 and its examples that $\phi_{1,1}$ gives an isomorphism of $\mathbb{R}$-algebras between $C(p, q)$ and $C(1,1) \otimes C(p-1, q-1)$. More than this, we need an isomorphism, denoted by $\eta_{\mathcal{K}}$, having an additional property (4.7.2).

Lemma 4.7.7. Suppose that $\mathcal{M}=(p, q)$ and $\mathcal{K}=\left(k_{+}, k_{-}\right)$are pairs of natural numbers satisfying $k_{+} \leq p, k_{-} \leq q, 1 \leq p, q, \mathcal{K} \neq(0,0)$, and $\mathcal{K} \neq \mathcal{M}$. Then, there exists an $\mathbb{R}$-algebra isomorphism $\eta_{\mathcal{K}}: C(p, q) \stackrel{\sim}{\rightarrow} C(1,1) \otimes C(p-1, q-1)$ satisfying:

$$
\eta_{\mathcal{K}}\left(V_{\mathcal{K}}\right)=\left\{\begin{array}{lll}
v_{1}^{+} \otimes 1 & \text { if } \Delta \mathcal{K} \equiv 0,1 \quad \bmod 4 \\
v_{1}^{-} \otimes 1 & \text { if } \Delta \mathcal{K} \equiv 2,3 \quad \bmod 4
\end{array}\right.
$$

Proof. Assume first $k_{+}<m_{+}$and $k_{-}>0$. We set $\mathcal{K}^{\prime}:=\left(k_{+}+1, k_{-}\right)$. (If this assumption is not satisfied, then it is easy to see $k_{+}>0$ and $k_{-}<m_{-}$. The proof goes similarly if we set $\mathcal{K}^{\prime}:=\left(k_{+}, k_{-}+1\right)$ in this case.)

Clearly, we have $k_{ \pm}^{\prime} \geq 1$ and $V_{k_{+}^{\prime}-1, k_{-}^{\prime}-1} v_{k_{-}}^{-}=V_{\mathcal{K}}$, and therefore it follows from Lemma 4.7.5 that 
We set

$$
\lambda_{\mathcal{K}^{\prime}}\left(V_{\mathcal{K}}\right)=\left\{\begin{array}{lll}
\varepsilon v_{k^{\prime}}^{+} & \text {if } \Delta \mathcal{K} \equiv 0,1 \quad \bmod 4 \\
\varepsilon v_{k^{\prime}}^{-} & \text {if } \Delta \mathcal{K} \equiv 2,3 \bmod 4
\end{array}\right.
$$

$$
\begin{aligned}
v & :=\left\{\begin{array}{lll}
\varepsilon v_{k^{\prime}}^{+}-v_{1}^{+} & \text {if } \Delta \mathcal{K} \equiv 0,1 \quad \bmod 4, \\
\varepsilon v_{k^{\prime}}^{-}-v_{1}^{-} & \text {if } \Delta \mathcal{K} \equiv 2,3 \bmod 4 .
\end{array}\right. \\
\eta_{\mathcal{K}} & :=\phi_{1,1} \circ \tau_{v} \circ \lambda_{\mathcal{K}^{\prime}} .
\end{aligned}
$$

Here, we interpret $\tau_{v}$ as the identity if $v=0$, which happens when $k_{+}^{\prime}=1$ and $\varepsilon=1$. Let us show that the isomorphism $\eta_{\mathcal{K}}: C(p, q) \stackrel{\sim}{\rightarrow} C(1,1) \otimes C(p-1, q-1)$ satisfies desired property (4.7.2).

Case i) $\Delta \mathcal{K} \equiv 0,1 \bmod 4$.

If $k_{+}^{\prime} \neq 1$, then $\tau_{v}$ exchanges $\varepsilon v_{k^{\prime}+}^{+}$and $v_{1}^{+}$. If $k_{+}^{\prime}=1$ and $\varepsilon=-1$, then $\tau_{v}$ changes the signature of $v_{1}^{+}$. If $k_{+}^{\prime}=1$ and $\varepsilon=1$, then $\tau_{v}=\mathrm{id}$. Thus, $\tau_{v} \circ \lambda_{\mathcal{K}^{\prime}}\left(V_{\mathcal{K}}\right)=v_{1}^{+}$ in all the cases. Applying $\phi_{1,1}$, we have:

$$
\phi_{1,1} \circ \tau_{v} \circ \lambda_{\mathcal{K}^{\prime}}\left(V_{\mathcal{K}}\right)=v_{1}^{+} \otimes 1 \quad \in C(1,1) \otimes C(p-1, q-1) .
$$

Hence, $\eta_{\mathcal{K}}$ satisfies (4.7.2).

Case ii) $\Delta \mathcal{K} \equiv 2,3 \bmod 4$.

The proof goes similarly.

Remark 4.7.8. If $\eta_{\mathcal{K}}$ is an isomorphism such that $\eta_{\mathcal{K}}\left(V_{\mathcal{K}}\right)=v_{1}^{\delta} \otimes 1$, then we can tell a priori the signature $\delta= \pm$ by (4.5.3).

Lemma 4.7.9. Suppose $\mathcal{M}=(p, q), \mathcal{K}=\left(k_{+}, k_{-}\right)$and $\mathcal{L}=\left(l_{+}, l_{-}\right)$satisfy $\mathcal{M}=\mathcal{K}+\mathcal{L}, \mathcal{K} \neq(0,0),(p, q), 1 \leq p$ and $\Delta \mathcal{M} \equiv \Delta \mathcal{L} \equiv 1 \bmod 2$. Then there exists an $\mathbb{R}$-algebra isomorphism $\xi_{\mathcal{L}}: C(p, q) \stackrel{\sim}{\rightarrow} C(\mathcal{X}) \otimes C(\mathcal{Y})$ such that

$$
\xi_{\mathcal{L}}\left(T_{\mathcal{L}}\right)=\left\{\begin{array}{lll}
\text { id } \otimes \tau_{1}^{+} & \text {if } \Delta \mathcal{M} \equiv 1 & \bmod 4 \\
\text { id } \otimes \tau_{1}^{-} & \text {if } \Delta \mathcal{M} \equiv 3 & \bmod 4 .
\end{array}\right.
$$

Here, $\mathcal{X}$ and $\mathcal{Y}$ are given by:

$$
\mathcal{X}=\left\{\begin{array}{lll}
(q, p-1) & \Delta \mathcal{K} \equiv 0 & \bmod 4, \\
(p-1, q) & \Delta \mathcal{K} \equiv 2 & \bmod 4 .
\end{array} \quad \mathcal{Y}=\left\{\begin{array}{lll}
(1,0) & \Delta \mathcal{M} \equiv 1 & \bmod 4 \\
(0,1) & \Delta \mathcal{M} \equiv 3 & \bmod 4
\end{array}\right.\right.
$$

Proof. It follows from $\mathcal{K} \neq(0,0)$, that at least one of $k_{+}$and $k_{-}$is non-zero. We shall give a proof in the case $k_{-} \geq 1$. The case $k_{-} \geq 1$ goes similarly. We set $\mathcal{K}^{\prime}:=\left(k_{+}+1, k_{-}\right)$. In light of $k_{ \pm}^{\prime} \geq 1$ and $\tau_{k_{+}^{\prime}}^{+} T_{\mathcal{M}-\mathcal{K}^{\prime}}=T_{\mathcal{L}}$, we have from Lemma 4.7.5:

$$
\lambda_{\mathcal{K}^{\prime}}\left(T_{\mathcal{L}}\right)=\left\{\begin{array}{lll}
\tau_{k_{-}^{\prime}}^{-} & \text {if } \Delta \mathcal{K} \equiv 0 & \bmod 4, \\
\tau_{k_{+}^{\prime}}^{+} & \text {if } \Delta \mathcal{K} \equiv 2 & \bmod 4
\end{array}\right.
$$


(i) Case $\Delta \mathcal{K} \equiv 0 \bmod 4$.

We set $v=v_{k_{-}^{\prime}}^{-}-v_{q}^{-}$and $\xi_{\mathcal{L}}:=\phi_{q, p-1} \circ \phi_{1,0} \circ \tau_{v} \circ \lambda_{\mathcal{K}^{\prime}}$. Here, we regard $\tau_{v}=\mathrm{id}$ if $v=0$ as before. In light that $\tau_{v}$ is an $\mathbb{R}$-algebra isomorphism of $C(p, q)$ that exchanges $v_{k^{\prime}}^{-}$and $v_{q}^{-}$, we have from Lemma 4.6.2:

$$
\begin{aligned}
& C(p, q) \underset{\lambda_{\mathcal{K}^{\prime}}}{\stackrel{\sim}{\longrightarrow}} C(p, q) \underset{\tau_{v}}{\stackrel{\sim}{\longrightarrow}} C(p, q) \underset{\phi_{1,0}}{\stackrel{\sim}{\longrightarrow}} C(q+1, p-1) \\
& T_{\mathcal{L}} \mapsto \tau_{k_{-}^{\prime}}^{-} \mapsto \tau_{q}^{-} \mapsto \tau_{q+1}^{+} .
\end{aligned}
$$

Applying furthermore the isomorphism $\phi_{q, p-1}$, we obtain

$$
\phi_{q, p-1}\left(\tau_{q+1}^{+}\right)=\left\{\begin{array}{lll}
\operatorname{id} \otimes \tau_{1}^{+} \in C(q, p-1) \otimes C(1,0) & \text { if } \Delta \mathcal{M} \equiv 1 & \bmod 4, \\
\operatorname{id} \otimes \tau_{1}^{-} \in C(q, p-1) \otimes C(0,1) & \text { if } \Delta \mathcal{M} \equiv 3 & \bmod 4 .
\end{array}\right.
$$

(ii) Case $\Delta \mathcal{K} \equiv 2 \bmod 4$.

We set $v=v_{k_{+}^{\prime}}^{+}-v_{p}^{+}$and $\xi_{\mathcal{L}}:=\phi_{p-1, q} \circ \tau_{v} \circ \lambda_{\mathcal{K}^{\prime}}$. Then the proof goes similarly.

Lemma 4.7.10. Suppose $\pi$ is any of the isomorphisms $\tau_{v}, \psi_{p, q}, \phi_{\mathcal{K}}, \lambda_{\mathcal{K}}, \eta_{\mathcal{K}}$ and $\xi_{\mathcal{K}}$ (see Lemmas 4.3.4, 4.6.1, 4.6.2, 4.7.2, 4.7.7 and 4.7.9, respectively for definition). Then

$$
\pi\left(a^{*}\right)=\pi(a)^{*} \text { for any } a \in C(p, q) .
$$

Here, for a matrix $A \in M(n, \mathbb{F})(\mathbb{F}=\mathbb{R}, \mathbb{C}, \mathbb{H}), A^{*}$ denotes the standard conjugation ${ }^{\bar{t}} \mathrm{~A}$.

Proof. It is sufficient to show (4.7.3) for the generators $\left\{v_{1}^{+}, \ldots, v_{p}^{+}, v_{1}^{-}, \ldots, v_{q}^{-}\right\}$.

1) For $\pi=\psi_{p, q}$, (4.7.3) follows straightforward from definition.

2) For $\pi=\phi_{\mathcal{K}}$, we first note $A^{*} A=A A^{*}=1$ if $A \in C(p, q)_{\text {mon }}$ (see Definition 4.3.1). Furthermore, $\phi_{\mathcal{K}}\left(v_{i}^{ \pm}\right) \in C(p, q)_{\text {mon }}$ by definition. Therefore, we have $\phi_{\mathcal{K}}\left(v_{i}^{ \pm}\right) \phi_{\mathcal{K}}\left(v_{i}^{ \pm}\right)^{*}=1$. On the other hand, it follows from $v_{i}^{ \pm *} v_{i}^{ \pm}=1$ that $\phi_{\mathcal{K}}\left(v_{i}^{ \pm *}\right) \phi_{\mathcal{K}}\left(v_{i}^{ \pm}\right)=1$ because $\phi_{\mathcal{K}}$ is an algebra isomorphism. Hence, we have proved $\phi_{\mathcal{K}}\left(v_{i}^{ \pm *}\right)=\phi_{\mathcal{K}}\left(v_{i}^{ \pm}\right)^{*}$. The cases $\pi=\tau_{v}$ and $\lambda_{\mathcal{K}}$ are similar.

3) For $\pi=\eta_{\mathcal{K}}$ and $\xi_{\mathcal{K}},(4.7 .3)$ holds because $\eta_{\mathcal{K}}$ and $\xi_{\mathcal{K}}$ are defined as the composition of $\tau, \phi$ and $\lambda$.

4.8. Proof of Proposition 4.4.4. In this subsection, we shall find the group structure of $C(p, q) \cap \operatorname{Aut}\left(J_{ \pm}\right)$(see Definition 4.5.4) and $G(p, q)$ (see Definition 4.3.1). Let us prove the next lemma:

Lemma 4.8.1. We have the following isomorphisms of $\mathbb{R}$-algebras:

1) $C_{\text {even }}(p, q) \simeq C(p, q)^{\tau_{1}^{-}} \simeq C(p, q-1) \quad(q>0)$. 
2) $C_{\text {even }}(p, q) \simeq C_{\text {even }}(q, p)$.

3) $G(p, q) \simeq G(q, p)$.

4) $G(p, q) \simeq C(p, q-1) \cap \operatorname{Aut}\left(J_{+}\right)$if $p$ is even and $q>0$.

5) $G(p, q) \simeq C(p, q-1) \cap \operatorname{Aut}\left(J_{-}\right)$if $q$ is even and $q>0$.

Proof. 1) We recall $C(p, q)^{\tau_{1}^{-}} \simeq C(p, q-1)$ and $C_{\text {even }}(p, q)=C(p, q)^{T_{p, q}}$ by Example 4.5.3. Applying $\phi_{0,1}$, we obtain

$$
C_{\text {even }}(p, q)=C(p, q)^{T_{p, q}} \underset{\phi_{0,1}}{\stackrel{\sim}{\longrightarrow}} C(p, q)^{\phi_{0,1}\left(T_{p, q}\right)} .
$$

Since $\phi_{0,1}\left(T_{p, q}\right)=\tau_{1}^{-}$by Lemma 4.6.2, the statement (1) follows.

$2)$ The statement is obvious in the case $(p, q)=(0,0)$. Suppose now $(p, q) \neq(0,0)$. Then, without loss of generality, we may assume $q \geq 1$. In view of $C_{\text {even }}(p, q)=$ $C(p, q)^{T_{p, q}}$, the isomorphism (2) is obtained as the composition:

$$
C(p, q)^{T_{p, q}} \underset{\phi_{0,1}}{\stackrel{\sim}{\longrightarrow}} C(p, q)^{\tau_{1}^{-}} \simeq C(p, q-1) \simeq C(p+1, q-1)^{\tau_{1}^{+}} \underset{\phi_{1,0}}{\sim} C(q, p)^{T_{q, p}} .
$$

Here, the last isomorphism follows from $\phi_{1,0}\left(\tau_{1}^{+}\right)=T_{q, p}$ by Lemma 4.6.2.

3) We denote by $\pi: C_{\text {even }}(p, q) \stackrel{\sim}{\rightarrow} C_{\text {even }}(q, p)$ the isomorphism constructed in (2). Then $\pi\left(a^{*}\right)=\pi(a)^{*}$ for any $a \in C_{\text {even }}(p, q)$ by Lemma 4.7.10. Therefore, to see $\pi\left({ }^{t} a\right)={ }^{t} \pi(a)$, it is sufficient to show that $\pi$ transfers $T_{p, 0}$ to $T_{0, p}$ because ${ }^{t} a=T_{p, 0}\left(a^{*}\right)$ by (4.5.6). Chasing the automorphisms in each step:

$$
\begin{gathered}
C_{\text {even }}(p, q) \underset{\phi_{0,1}}{\stackrel{\sim}{\longrightarrow}} C(p, q)^{\tau_{1}^{-}} \simeq C(p+1, q-1)^{\tau_{1}^{+}} \underset{\phi_{1,0}}{\stackrel{\sim}{\longrightarrow}} C_{\text {even }}(q, p) \\
\tau_{1}^{+} \cdots \tau_{p}^{+} \mapsto \tau_{1}^{+} \cdots \tau_{p}^{+} \mapsto \quad \tau_{2}^{+} \cdots \tau_{p+1}^{+} \stackrel{\mapsto}{\mapsto} \tau_{1}^{-} \cdots \tau_{p}^{-}
\end{gathered}
$$

by using Lemma 4.6.2, we see $\pi$ transfers $T_{p, 0}$ to $T_{0, p}$. Hence (3) follows.

4) We notice $J_{+} \in C_{\text {even }}(p, q)$ if $p$ is even. Then, a simple computation shows that the isomorphism $C_{\text {even }}(p, q) \stackrel{\sim}{\rightarrow} C(p, q-1)$ constructed in (1) sends $J_{+} \in$ $C_{\text {even }}(p, q)$ to $J_{+} \in C(p, q-1)$. Hence, the statement (4) follows.

5) The proof is similar to (4).

Remark 4.8.2. Lemma 4.8.1 implies, in particular, that the groups $C(p, q) \cap$ $\operatorname{Aut}\left(J_{+}\right)$and $C(p, q) \cap \operatorname{Aut}\left(J_{-}\right)$are isomorphic if $p$ is even and $q$ is odd. In fact, these two groups coincide (see Remark 4.5.6).

In light of Lemma 4.8.1, we can tell the group structure of $G(p, q)$ in the case $p q$ is even by finding the group structure of $C(p, q-1) \cap \operatorname{Aut}\left(J_{ \pm}\right)$. This is given in the following proposition:

Proposition 4.8.3. For $p, q \geq 1$, we set $\alpha$ as in the table below and $n:=$ $2^{(p+q-\alpha) / 2}$. Then, the group $C(p, q) \cap \operatorname{Aut}\left(J_{+}\right)$is isomorphic to one of the following Lie groups. 


\begin{tabular}{c|lllc}
$C(p, q) \cap \operatorname{Aut}\left(J_{+}\right)$ & $p$ & $\bmod 4$ & $\alpha$ & $p-q-1$ \\
\hline$O(n, n)^{2}$ & 0,1 & 3 & 0 \\
$S p(n, \mathbb{R})^{2}$ & 2,3 & & \pm 1 \\
\hline$O(n, n)$ & 0,1 & 2 & \pm 2 \\
$S p(n, \mathbb{R})$ & 2,3 & & \pm 3 \\
\hline$U(n, n)$ & $a n y$ & 3 & \multirow{2}{*}{4}
\end{tabular}

Likewise, the group $C(p, q) \cap \operatorname{Aut}\left(J_{-}\right)$is given, if we replace the condition $p$ $\bmod 4$ by $q-1 \bmod 4$ in the above table.

Proof. Combining the isomorphism $\psi_{1,1} \stackrel{\sim}{\rightarrow} M(2, \mathbb{R})$ (see Lemma 4.6.1) with $\eta_{p, 0}$ : $C(p, q) \stackrel{\sim}{\rightarrow} C(1,1) \otimes C(p-1, q-1)$ (see Lemma 4.7.7), we obtain an isomorphism $\iota: C(p, q) \stackrel{\sim}{\rightarrow} M(2, \mathbb{R}) \otimes C(p-1, q-1)$ satisfying

$$
\iota\left(J_{+}\right)=\left\{\begin{array}{lll}
\left({ }^{1}-1\right) \otimes 1 & \text { if } p \equiv 0,1 \quad \bmod 4 \\
\left({ }_{1}-1\right) \otimes 1 & \text { if } p \equiv 2,3 \quad \bmod 4 .
\end{array}\right.
$$

Case i) $p-q \equiv 0,2,3 \bmod 4$.

Combining the isomorphism $C(p-1, q-1) \simeq M(n, \mathbb{F})(\mathbb{F}=\mathbb{R}, \mathbb{C}$ or $\mathbb{H})$ given in Proposition 4.4.1, we find that the group $C(p, q) \cap \operatorname{Aut}\left(J_{+}\right)$is isomorphic to $O(n, n), S p(n, \mathbb{R})(\mathbb{F}=\mathbb{R}) ;$ or $S p(n, n), O^{*}(4 n)(\mathbb{F}=\mathbb{H})$. It is isomorphic to $U(n, n)$ (independently of $p \bmod 4)$ if $\mathbb{F}=\mathbb{C}$.

Case ii) $p-q \equiv 1 \bmod 4$.

We note $C(p, q) \simeq M(2 n, \mathbb{F})^{2}(\mathbb{F}=\mathbb{R}$ or $\mathbb{H})$. The proof goes similarly.

This completes the proof for $C(p, q) \cap \operatorname{Aut}\left(J_{+}\right)$. For the group structure of $C(p, q) \cap \operatorname{Aut}\left(J_{-}\right)$, we use $\eta_{0, q}$ instead of $\eta_{p, 0}$.

Owing to Lemma 4.8.1 (4) and (5), this Proposition completes the proof of Proposition 4.4.4 in the case $p q$ is even.

Next, let us consider the case where both $p$ and $q$ are odd. Owing to Lemma 4.5.5, Proposition 4.4.4 in this case is reduced to the group structure of $C_{\text {even }}(p, q) \cap$ $\operatorname{Aut}\left(J_{ \pm}\right)$. Here is a result:

Proposition 4.8.4. Suppose $p q$ is odd. We set $n=2^{(p+q-\alpha) / 2}$ and $\alpha \in\{2,4\}$ is given in the table below. Then, the group $G(p, q) \simeq C_{\text {even }}(p, q) \cap \operatorname{Aut}\left(J_{ \pm}\right)$is 
isomorphic to the following Lie groups:

\begin{tabular}{|c|c|c|c|}
\hline$C_{\text {even }}(p, q) \cap \operatorname{Aut}\left(J_{ \pm}\right)$ & $p-q \bmod 8$ & $p+q \bmod 8$ & $\alpha$ \\
\hline$G L(n, \mathbb{R})$ & 0 & & 2 \\
\hline$G L(n, \mathbb{H})$ & 4 & \pm 2 & 4 \\
\hline$O(n, \mathbb{C})$ & \pm 2 & 0 & 2 \\
\hline
\end{tabular}

Proof. We first note that the isomorphism $\phi_{0,1}: C_{\text {even }}(p, q) \stackrel{\sim}{\rightarrow} C(p, q-1)$ transfers the automorphism $T_{p, 0}$ of $C_{\text {even }}(p, q)$ to the automorphism $T_{p, 0}$ of $C(p, q-1)$ (see Lemmas 4.8.1(1) and 4.6.2(2)(iv)). Now, applying Lemma 4.7.9 to the case where $\mathcal{M}=(p, q-1), \mathcal{L}=(p, 0)$ and $\mathcal{K}=(0, q-1)$, we obtain an isomorphism

$$
\xi_{p, 0}: C(p, q-1) \stackrel{\sim}{\rightarrow} C(\mathcal{X}) \otimes C(\mathcal{Y})
$$

satisfying

$$
\xi_{p, 0}\left(T_{p, 0}\right)=\left\{\begin{array}{lll}
\mathrm{id} \otimes \tau_{1}^{+} & \text {if } p-q \equiv 0 & \bmod 4, \\
\mathrm{id} \otimes \tau_{1}^{-} & \text {if } p-q \equiv 2 & \bmod 4 .
\end{array}\right.
$$

Here, we have set

$$
\mathcal{X}:=\left\{\begin{array}{rrrr}
(p, q-2) & q \equiv 1 \quad \bmod 4, \\
(p-1, q-1) & q \equiv 3 & \bmod 4,
\end{array} \quad \mathcal{Y}:=\left\{\begin{array}{rrr}
(1,0) & p-q \equiv 0 & \bmod 4 \\
(0,1) & p-q \equiv 2 & \bmod 4
\end{array}\right.\right.
$$

Case i) $p-q \equiv 0 \bmod 4$.

We note $\xi_{p, 0}\left(T_{p, 0}\right)=\mathrm{id} \otimes \tau_{1}^{+}$.

In this case, $C(\mathcal{Y})=C(1,0) \simeq \mathbb{R} \oplus \mathbb{R}$ and $\tau_{1}^{+}$acts on $C(\mathcal{Y})$ as $(a, b) \mapsto(b, a)$. We now assert:

Claim. $C(\mathcal{X}) \simeq M(n, \mathbb{F})$. Here, $\mathbb{F}$ is given by

$$
\mathbb{F}=\left\{\begin{array}{lll}
\mathbb{R} & \text { if } p-q \equiv 0 & \bmod 8 \\
\mathbb{H} & \text { if } p-q \equiv 4 & \bmod 8
\end{array}\right.
$$

This claim is a direct consequence of Proposition 4.4.1. Combining the isomorphism $\xi_{p, 0}($ see $(4.8 .1))$ with $C(\mathcal{X}) \simeq M(n, \mathbb{F})$ and $C(\mathcal{Y}) \simeq \mathbb{R} \oplus \mathbb{R}$, we obtain an isomorphism $\iota: C_{\text {even }}(p, q) \simeq M(n, \mathbb{F}) \oplus M(n, \mathbb{F})$. In light of ${ }^{t} a=T_{p, 0}\left(a^{*}\right)$ for $a \in C_{\text {even }}(p, q)$ (see (4.5.6)), we have $\iota\left({ }^{t} a\right)=\left(B^{*}, A^{*}\right)$ if we write $\iota(a)=(A, B)$. Hence, the following conditions on $a \in C_{\text {even }}(p, q)$ are equivalent:

$$
\begin{aligned}
a \in G(p, q) & \Longleftrightarrow{ }^{t} a a=1 \\
& \Longleftrightarrow\left(B^{*}, A^{*}\right)(A, B)=(1,1) \\
& \Longleftrightarrow B^{*}=A^{-1} .
\end{aligned}
$$

This shows that the group $G(p, q)$ is isomorphic to the general linear group $G L(n, \mathbb{F})$, where $\mathbb{F}=\mathbb{R}$ or $\mathbb{H}$ as in (4.8.2). 
Case ii) $p-q \equiv 2 \bmod 4$.

We note $\xi_{p, 0}\left(T_{p, 0}\right)=\mathrm{id} \otimes \tau_{1}^{-}$. In this case, $C(\mathcal{Y})=C(0,1) \simeq \mathbb{C}$, and $\tau_{1}^{-}$acts on $C(\mathcal{Y})$ as complex conjugation. It follows from our assumption $q \equiv 1 \bmod 2$ and $p-q \equiv 2 \bmod 4$ that $p+q \equiv 0 \bmod 4$, and therefore $p+q \bmod 8$ is either 0 or 4 .

Subcase ii-a) $p+q \equiv 0 \bmod 8$.

If $q \equiv 1 \bmod 4, \Delta \mathcal{X}=q-p=2 q-(p+q) \equiv 2 \bmod 8$. If $q \equiv 3 \bmod 4$, $\Delta \mathcal{X}=p-q=(p+q)-2 q \equiv 2 \bmod 8$. Hence, in either case, $C(\mathcal{X})$ is isomorphic to $M(n, \mathbb{R})$ by Proposition 4.4.1, and thus we obtain an isomorphism $\iota: C_{\text {even }}(p, q) \stackrel{\sim}{\rightarrow} M(n, \mathbb{C})$. Then by using $T_{p, 0}\left(a^{*}\right)={ }^{t} a$ again and $\xi_{p, 0}\left(T_{p, 0}\right)=\operatorname{id} \otimes \tau_{1}^{-}$, we have

$$
\iota\left({ }^{t} a\right)=\iota\left(T_{p, 0}\left(a^{*}\right)\right)=\overline{\iota(a)^{*}}={ }^{t} \iota(a) .
$$

This shows that the group, $G(p, q)$ is isomorphic to $O(n, \mathbb{C})$.

Subcase ii-b) $p+q \equiv 4 \bmod 8$.

Similarly to the subcase (ii-a), we have an isomorphism $C(\mathcal{X}) \simeq M(n, \mathbb{H})$. Via the isomorphism $M(n, \mathbb{H}) \otimes \mathbb{C} \stackrel{\sim}{\rightarrow} M(2 n, \mathbb{C})$ defined by

$$
(P+Q j) \otimes 1 \mapsto\left(\frac{P}{\bar{Q}} \frac{-Q}{P}\right), \quad 1 \otimes z \mapsto\left(\begin{array}{cc}
z I_{n} & \\
& z I_{n}
\end{array}\right),
$$

id $\otimes \tau_{1}^{-}$induces the involution of $M(2 n, \mathbb{C})$ given by

$$
z\left(\frac{P}{\bar{Q}} \overline{-Q} \bar{P}\right) \mapsto \bar{z}\left(\frac{P}{\bar{Q}} \frac{-Q}{\bar{P}}\right)=\overline{z\left(\begin{array}{cc}
\bar{P} & -\bar{Q} \\
Q & P
\end{array}\right)}
$$

that is,

$$
\left(\begin{array}{cc}
A & B \\
C & D
\end{array}\right) \mapsto\left(\begin{array}{cc}
\bar{D} & -\bar{C} \\
-\bar{B} & \bar{A}
\end{array}\right) .
$$

Therefore, the resulting isomorphism $\iota: C_{\text {even }}(p, q) \stackrel{\sim}{\rightarrow} M(2 n, \mathbb{C})$ has the following property:

$$
\iota\left(T_{p, 0}(a)\right)=\left(\begin{array}{cc}
\bar{D} & -\bar{C} \\
-\bar{B} & \bar{A}
\end{array}\right),
$$

if we write $\iota(a)=\left(\begin{array}{cc}A & B \\ C & D\end{array}\right) \in M(2 n, \mathbb{C})$ for $a \in C_{\text {even }}(p, q)$. Then, by using ${ }^{t} a=$ $T_{p, 0}\left(a^{*}\right)$, we obtain

$$
\iota\left({ }^{t} a\right)=\left(\begin{array}{cc}
{ }^{t} D & -{ }^{t} B \\
-{ }^{t} C & { }^{t} A
\end{array}\right)=J_{n}^{-1}\left({ }^{t} \iota(a)\right) J_{n},
$$

where $J_{n}:=\left({ }_{I_{n}}-I_{n}\right) \in M(2 n, \mathbb{C})$. Hence, for $a \in C_{\text {even }}(p, q)$,

$$
a \in G(p, q) \Longleftrightarrow{ }^{t} a a=1 \Longleftrightarrow{ }^{t} \iota(a) J_{n} \iota(a)=J_{n},
$$

and thus $G(p, q)$ is isomorphic to $S p(n, \mathbb{C})$. This completes the proof of Proposition in all the cases. 
4.9. Proof of Theorem 4.2.1. This subsection gives a proof of Theorem 4.2.1. We have already seen $d(G)=d(H)+d(L)$ (the condition (3.3.3)(b)) in Table 4.2.

Let us verify the condition (3.3.3)(a). We write $\mathfrak{g}$ for the Lie algebra of $G(p, q)$. Then, $\mathfrak{g}=\left\{X \in C_{\text {even }}(p, q):{ }^{t} X+X=0\right\}$, and $X \mapsto-X^{*}$ defines a Cartan involution of $\mathfrak{g}$. Let $\mathfrak{g}=\mathfrak{k}+\mathfrak{p}$ the corresponding Cartan decomposition, which then induces a Cartan decomposition $\mathfrak{l}=(\mathfrak{l} \cap \mathfrak{k})+(\mathfrak{l} \cap \mathfrak{p})$ of the Lie algebra $\mathfrak{l}$ of $\operatorname{Spin}(p, q)$.

Lemma 4.9.1. Suppose $q \geq 1$, and $(\pi, W)$ is a representation of the $\mathbb{R}$-algebra $C_{\text {even }}(1, q) . \quad$ We note $\mathfrak{l}=\mathfrak{s p i n}(1, q) \subset C_{\text {even }}(1, q)$. Then $\pi(X) \in \operatorname{End}(W)$ is invertible for any non-zero $X \in \mathfrak{l} \cap \mathfrak{p}$.

Proof. Retain the notation as in Section 4.5. We set $X_{0}:=v_{1}^{+} v_{1}^{-}$. Then $X_{0} \in \mathfrak{p}$ because $X_{0}{ }^{*}=\left(v_{1}^{-}\right)^{*}\left(v_{1}^{+}\right)^{*}=X_{0}$. As $X_{0}^{2}=1$, we have $\pi\left(X_{0}\right)^{2}=\pi(1)=$ id because $\pi$ is a representation of an $\mathbb{R}$-algebra. Hence, $\pi\left(X_{0}\right)$ is invertible. For $t \in \mathbb{R}$, we set

$$
x(t):=\exp \left(t X_{0}\right)=(\cosh t)+(\sinh t) v_{1}^{+} v_{1}^{-}=v_{1}^{+}\left((\cosh t) v_{1}^{+}+(\sinh t) v_{1}^{-}\right) .
$$

Then, $x(t) \in \operatorname{Spin}(1, q)$ by Definition 4.3 .1 , and $\{x(t): t \in \mathbb{R}\}$ forms a oneparameter subgroup of $\operatorname{Spin}(1, q)$ consisting of hyperbolic elements. Since $\mathbb{R}$ $\operatorname{rank}(\operatorname{Spin}(1, q))=1$, any non-zero $X \in \mathfrak{l} \cap \mathfrak{p}$ can be written as

$$
X=\operatorname{Ad}(l) t X_{0}
$$

for some $l \in L \cap K$ and some non-zero $t \in \mathbb{R}$. Hence, $\pi(X)^{2}=t^{2}$ id and we have seen $\pi(X)$ is invertible.

Then, the proof of Theorem 4.2.1 will be completed if we show the following:

Proposition 4.9.2. Each triple of Lie groups $(G, H, L)$ in the following table satisfies the condition (3.3.3)(a), or equivalently, $L \pitchfork H$ in $G$. Here $\alpha \in\{0,1,2,3\}$ is given in the table, and $n=2^{4 r-\alpha}$.

\begin{tabular}{|ccc|cc|}
\hline$G$ & $H$ & $L$ & $W$ & $\alpha$ \\
\hline$G L(n, \mathbb{R})$ & $G L(n-1, \mathbb{R})$ & $\operatorname{Spin}(1,8 r+1)$ & $\mathbb{R}^{n}$ & 0 \\
$S p(n, \mathbb{R})$ & $S p(n-1, \mathbb{R})$ & $\operatorname{Spin}(1,8 r+2)$ & $\mathbb{R}^{2 n}$ & 0 \\
$S p(n, \mathbb{C})$ & $S p(n-1, \mathbb{C})$ & $\operatorname{Spin}(1,8 r+3)$ & $\mathbb{C}^{2 n}$ & 0 \\
$S p(n, n)$ & $S p(n-1, n)$ & $\operatorname{Spin}(1,8 r+4)$ & $\mathbb{H}^{2 n}$ & 0 \\
$G L(n, \mathbb{H})$ & $G L(n-1, \mathbb{H})$ & $\operatorname{Spin}(1,8 r+5)$ & $\mathbb{H}^{n}$ & 1 \\
$O^{*}(2 n)$ & $O^{*}(2 n-2)$ & $\operatorname{Spin}(1,8 r+6)$ & $\mathbb{H}^{n}$ & 2 \\
$O(n, \mathbb{C})$ & $O(n-1, \mathbb{C})$ & $\operatorname{Spin}(1,8 r+7)$ & $\mathbb{C}^{n}$ & 3 \\
$O(n, n)$ & $O(n-1, n)$ & $\operatorname{Spin}(1,8 r+8)$ & $\mathbb{R}^{2 n}$ & 3 \\
\hline
\end{tabular}

Table 4.9 
Proof. Let $W$ be the linear space as a given in Table 4.9, and $\left\{e_{i}\right\}$ the standard basis of $W$. We note that the subgroup $H$ in Table 4.9 is given by

$$
H=\left\{g \in G: g\left(e_{1}\right)=e_{1}\right\},
$$

and thus its Lie algebra $\mathfrak{h}$ is given by $\mathfrak{h}=\left\{X \in \mathfrak{g}: X e_{1}=0\right\}$. In particular, any element in $\mathfrak{h}$ is not invertible in $\operatorname{End}(W)$.

Let $\mathfrak{a} \subset \mathfrak{p}$ be a maximally split abelian subalgebra of $\mathfrak{g}$. Then $\mathfrak{a}_{H}$ consists of non-invertible elements in $\operatorname{End}(W)$, while any non-zero element of $\mathfrak{a}_{L}$ is invertible by Lemma 4.9.1. Thus we have

$$
\mathfrak{a}(L) \cap \mathfrak{a}(H)=0 .
$$

The condition (3.3.3)(a) is proved. Thus, Proposition 4.9.2 is proved.

4.10. Compact Clifford-Klein form of $S O(8, \mathbb{C}) / S O(7,1)$. This subsection proves the case 10 of Theorem 2.2.1, that is:

Theorem 4.10.1. There exists a compact Clifford-Klein form of the symmetric space $S O(8, \mathbb{C}) / S O(7,1)$.

The main machinery for the proof here is the Clifford algebra associated to indefinite quadratic forms and Theorem 3.3.3. We shall show that the triple of Lie groups $(G, H, L):=(O(8, \mathbb{C}), O(7,1), \operatorname{Spin}(7, \mathbb{C}))$ satisfies the conditions (3.3.3)(a) and (b).

First, the embedding $L \subset G$ is obtained as a complexification of the compact real form $\operatorname{Spin}(7) \subset G(0,7) \simeq O(8)$ (see Table 4.4.3).

In order to verify the condition (3.3.3)(a), let us compute $\mathfrak{a}_{L}$ in $\mathfrak{a}$. To do this, we first write explicitly the group isomorphism $G(0,7) \simeq O(8)$, which is obtained as the restriction of the $\mathbb{R}$-algebra isomorphism $\iota: C_{\text {even }}(0,7) \stackrel{\sim}{\rightarrow} M(8, \mathbb{R})$ to $G(0,7)$. We recall from Proposition 4.4.1 that $\iota$ is defined as the composition of the following isomorphism:

$$
\begin{gathered}
C_{\text {even }}(0,7) \underset{\phi_{0,1}}{\stackrel{\sim}{\longrightarrow}} C(0,6) \underset{\phi_{0,3}}{\stackrel{\sim}{\longrightarrow}} C(3,3) \underset{\phi_{1,1}}{\stackrel{\sim}{\longrightarrow}} C(1,1) \otimes C(1,1) \otimes C(1,1) \\
\underset{\psi_{1,1}}{\sim} M(2, \mathbb{R}) \otimes M(2, \mathbb{R}) \otimes M(2, \mathbb{R}) \simeq M(8, \mathbb{R}) .
\end{gathered}
$$

Let $E_{i j}$ be the matrix unit, and we choose a Cartan subalgebra of the Lie algebra $\mathfrak{o}(8)$ in $M(8, \mathbb{R}), \mathfrak{t}=\bigoplus_{k=1}^{4} \mathbb{R} f_{k}$ by taking

$$
f_{k}:=E_{2 k, 2 k-1}-E_{2 k-1,2 k} \quad(k=1,2,3,4) .
$$

Then $\mathfrak{a}:=\sqrt{-1} \mathfrak{t}$ becomes a maximally split abelian subalgebra of $\mathfrak{o}(8, \mathbb{C})=$ $\mathfrak{o}(8)+\sqrt{-1} \mathfrak{o}(8)$. We shall identify $\mathfrak{t}$ with $\mathbb{R}^{4}$ by means of the basis $f_{1}, \ldots, f_{4}$. We assert: 
Claim: $\quad \mathfrak{a}(L)=\sqrt{-1} \bigcup_{\substack{\varepsilon_{i} \in\{1,-1\} \\ \varepsilon_{1} \varepsilon_{2} \varepsilon_{3} \varepsilon_{4}=1}}\left\{{ }^{t}(a, b, c, d): \varepsilon_{1} a+\varepsilon_{2} b+\varepsilon_{3} c+\varepsilon_{4} d=0\right\}$.

To see Claim, we set

$$
x_{1}:={ }^{t}(1,1,1,1), \quad x_{2}:={ }^{t}(1,-1,1,-1), \quad x_{3}:={ }^{t}(1,-1,-1,1) .
$$

Then, $\iota^{-1}\left(x_{k}\right)(k=1,2,3)$ is computed by using Lemmas 4.6.1, 4.6.2 and 4.8.1(1) as follows:

$$
\begin{aligned}
& M(8, \mathbb{R}) \simeq \quad M(2, \mathbb{R}) \otimes M(4, \mathbb{R}) \quad \simeq C(1,1) \otimes C(1,1) \otimes C(1,1) \\
& x_{1} \mapsto \quad\left({ }_{1}^{-1}\right) \otimes \operatorname{diag}(1,1,1,1) \mapsto \quad v_{1}^{-} \otimes 1 \otimes 1 \\
& x_{2} \mapsto\left({ }_{1}{ }^{-1}\right) \otimes \operatorname{diag}(1,-1,1,-1) \mapsto \quad v_{1}^{-} \otimes v_{1}^{+} \otimes 1 \\
& x_{3} \mapsto\left(\begin{array}{c}
1 \\
1
\end{array}{ }^{-1}\right) \otimes \operatorname{diag}(1,-1,-1,1) \mapsto \quad v_{1}^{-} \otimes v_{1}^{+} \otimes v_{1}^{+} \\
& \stackrel{\sim}{\rightarrow} C(3,3) \stackrel{\sim}{\rightarrow} C(0,6) \stackrel{\sim}{\rightarrow} C_{\text {even }}(0,7) \\
& \mapsto v_{1}^{-} \mapsto v_{1}^{-} \mapsto v_{1}^{-} v_{2}^{-} \\
& \mapsto v_{1}^{+} v_{2}^{+} \mapsto v_{4}^{-} v_{5}^{-} \mapsto v_{5}^{-} v_{6}^{-} \\
& \mapsto v_{1}^{-} v_{2}^{-} v_{3}^{+} \mapsto v_{3}^{-} v_{6}^{-} \mapsto v_{4}^{-} v_{7}^{-}
\end{aligned}
$$

Then, $v_{1}^{-} v_{2}^{-}, v_{5}^{-} v_{6}^{-}, v_{4}^{-} v_{7}^{-}$are elements of the Lie algebra $\mathfrak{l}$ of $L=\operatorname{Spin}(7)$, as it follows from

$$
\exp \left(t v_{1}^{-} v_{2}^{-}\right)=\cos t+(\sin t)\left(v_{1}^{-} v_{2}^{-}\right)=v_{1}^{-}\left(-(\cos t) v_{1}^{-}+(\sin t) v_{2}^{-}\right)
$$

and alike, where we used $\left(v_{1}^{-} v_{2}^{-}\right)^{2}=-1$. Furthermore, since $v_{1}^{-} v_{2}^{-}, v_{5}^{-} v_{6}^{-}$and $v_{4}^{-} v_{7}^{-}$mutually commute, we conclude that $\mathbb{R} x_{1} \oplus \mathbb{R} x_{2} \oplus \mathbb{R} x_{3}$ is a Cartan subalgebra of $\mathfrak{l}$ because $\operatorname{rank} L=3$. Hence, $\sqrt{-1}\left(\mathbb{R} x_{1} \oplus \mathbb{R} x_{2} \oplus \mathbb{R} x_{3}\right)$ is a maximally split abelian subspace of $\mathfrak{l}_{\mathbb{C}}$. Since this subspace is contained in $\mathfrak{a}, \mathfrak{a}(L)$ is given

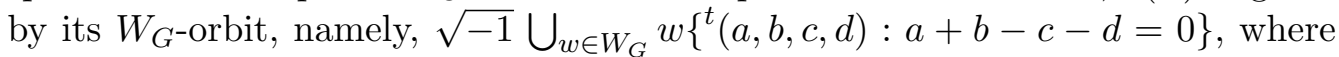
$W_{G}$ is the Weyl group of $O(8, \mathbb{C})$. Hence Claim is proved.

On the other hand, it is easy to see

$$
\begin{aligned}
\mathfrak{a}(H) & =W_{G}\left(\sqrt{-1} \mathbb{R} f_{1}\right) \\
& =\sqrt{-1} \mathbb{R}^{t}(1,0,0,0) \cup \sqrt{-1} \mathbb{R}^{t}(0,1,0,0) \cup \sqrt{-1} \mathbb{R}^{t}(0,0,1,0) \cup \sqrt{-1} \mathbb{R}^{t}(0,0,0,1) .
\end{aligned}
$$

Thus, $\mathfrak{a}(L) \cap \mathfrak{a}(H)=0$, and therefore the condition (3.3.3)(a) is proved. Since the condition (3.3.3)(b) is clear from $d(G)=28=7+21=d(L)+d(H)$, we conclude that the symmetric space $G / H=O(8, \mathbb{C}) / O(7,1)$ (therefore, $S O(8, \mathbb{C}) / S O(7,1)$ also) admits a compact Clifford-Klein form by Theorem 3.3.3. Hence, Theorem 4.10 .1 is proved.

\section{TANGential SPACE FORM PROBLEM}

In this section, we introduce the tangential homogeneous space $G_{\theta} / H_{\theta}$ associated to a homogeneous space $G / H$ of reductive type (see Definition 5.1.2). This 
is also a symmetric space if $G / H$ is symmetric. We shall see that most of the results on discontinuous groups for $G / H$ can be generalized or strengthened to those for the tangential homogeneous spaces $G_{\theta} / H_{\theta}$. Furthermore, if Conjecture 3.3.10 is true, then the existence of compact Clifford-Klein forms of $G / H$ implies that of $G_{\theta} / H_{\theta}$. Thus, the solution to Problem A for $G_{\theta} / H_{\theta}$ might be a good approximate to that of the original problem $G / H$, even though there is no general theory that bridges directly between $G / H$ and $G_{\theta} / H_{\theta}$ for Problem A.

Because the 'non-compact part' of $G_{\theta}$ is abelian, a simple and detailed study becomes possible for $G_{\theta} / H_{\theta}$. In particular, we shall prove Theorem 1.1.6, which gives an answer to the tangential version of our space form conjecture (Conjecture 4.1.2).

5.1. Tangential symmetric space. Let $G$ be a linear reductive Lie group with a Cartan involution $\theta$. We write the Cartan decomposition as $G=(\exp \mathfrak{p}) K$, and form a semidirect group

$$
G_{\theta}:=K \ltimes_{\mathrm{Ad}} \mathfrak{p}
$$

with multiplication: $\left(k_{1}, X_{1}\right)\left(k_{2}, X_{2}\right):=\left(k_{1} k_{2}, X_{1}+\operatorname{Ad}\left(k_{1}\right) X_{2}\right)$. We say $G_{\theta}$ is the Cartan motion group of $G$.

We define an involutive automorphism $\bar{\theta}$ of the Cartan motion group $G_{\theta}=$ $K \ltimes \mathfrak{p}$ by

$$
\bar{\theta}: G_{\theta} \rightarrow G_{\theta}, \quad(k, X) \mapsto(k,-X) .
$$

By using the Cartan decomposition $G=(\exp \mathfrak{p}) K$, we define a diffeomorphism

$$
\Phi_{\theta}: G \rightarrow G_{\theta}, \quad e^{X} k \mapsto(k, X) .
$$

Clearly, we have:

$$
\bar{\theta} \Phi_{\theta}=\Phi_{\theta} \theta
$$

Remark 5.1.1. In defining $\Phi_{\theta}$, we have used the Cartan decomposition $G=$ $(\exp \mathfrak{p}) K$ instead of $G=K \exp \mathfrak{p}$. The advantage of our definition is that the following natural formula holds:

$$
\Phi_{\theta}\left(k_{1}\right) \Phi_{\theta}(g) \Phi_{\theta}\left(k_{2}\right)=\Phi_{\theta}\left(k_{1} g k_{2}\right) \quad\left(g \in G, k_{1}, k_{2} \in K\right) .
$$

$\Phi_{\theta}$ is not a group homomorphism, and in general, the image $\Phi_{\theta}(H)$ of a subgroup $H$ is not a subgroup of $G_{\theta}$. However, if $H$ is a connected $\theta$-stable subgroup, then $\Phi_{\theta}(H)$ becomes a $\bar{\theta}$-stable subgroup of $G_{\theta}$. In this case, $H$ is a reductive Lie group with the Cartan involution $\left.\theta\right|_{H}$, and the Cartan motion group $H_{\theta}$ is naturally isomorphic to $\Phi_{\theta}(H)$. Thus, we can and do regard $H_{\theta}$ as a closed subgroup of $G_{\theta}$. The same argument is valid if we allow $H$ to have finitely many connected components. 
Definition 5.1.2. We say the homogeneous space $G_{\theta} / H_{\theta}$ is the tangential homogeneous space of $G / H$. Furthermore, if $G / H$ is a symmetric space, so is $G_{\theta} / H_{\theta}$, which we say the tangential symmetric space of $G / H$.

This section studies Problem A for the tangential symmetric space $G_{\theta} / H_{\theta}$. In contrast to a reductive symmetric space $G / H$, we can prove affirmatively a tangential version of Conjecture 3.3.10 (see Theorem 5.3.1). In particular, this enables us to solve completely the tangential analog of the space form problem, for which the original version has not yet found a final answer (see Section 4.1).

5.2. Discontinuous groups for tangential homogeneous spaces. This subsection reveals a strong resemblance on discontinuous group between a reductive homogeneous space $G / H$ and its tangential homogeneous space $G_{\theta} / H_{\theta}$. The proof for $G_{\theta} / H_{\theta}$ is easier or analogous to those for $G / H$.

1) $G_{\theta} / H_{\theta}$ is diffeomorphic to $G / H$. Furthermore, both of them are isomorphic to $K \times_{K \cap H}(\mathfrak{q} \cap \mathfrak{p})$ as $K$-equivariant fiber bundles over the compact homogeneous space $K / K \cap H$ (see (3.2.7) for notation).

2) $L \pitchfork H$ in $G \Longleftrightarrow L_{\theta} \pitchfork H_{\theta}$ in $G_{\theta}$ (see Lemmas 3.2.1 and 5.3.6).

3) $L \sim H$ in $G \Longleftrightarrow L_{\theta} \sim H_{\theta}$ in $G_{\theta}$.

4) $d(G)=d\left(G_{\theta}\right), \quad d(H)=d\left(H_{\theta}\right)$.

5) $L \backslash G / H$ is compact $\Longleftrightarrow L_{\theta} \backslash G_{\theta} / H_{\theta}$ is compact, provided $L$ and $H$ are $\theta$-stable connected subgroups such that $L \pitchfork H$ in $G$, or equivalently, $L_{\theta} \pitchfork H_{\theta}$ in $G_{\theta}$ (see Lemmas 3.3.4 and 5.3.5).

6) If $G / H$ is in Table 3.3 , both $G / H$ and $G_{\theta} / H_{\theta}$ admit compact CliffordKlein forms.

7) If $G / H$ satisfies the assumption of Theorem 3.5.1, neither $G / H$ nor $G_{\theta} / H_{\theta}$ admits compact Clifford-Klein forms.

8) If $\mathbb{R}$ - $\operatorname{rank} G=\mathbb{R}$-rank $H$, then neither $G / H$ nor $G_{\theta} / H_{\theta}$ admits infinite discontinuous groups (cf. Theorem 3.2.5).

Though $G / H$ is diffeomorphic to $G_{\theta} / H_{\theta}$, discontinuous groups for $G / H$ are quite different from those for $G_{\theta} / H_{\theta}$ as abstract groups in general. In fact, we shall deal essentially with abelian discontinuous subgroup in the case of a Cartan motion group $G_{\theta}$, as is seen in Bieberbach's theorem on discrete subgroups in $O(n) \ltimes \mathbb{R}^{n}$.

The next subsection gives a result that reduces Problem A to the existence problem of a certain connected subgroup (see Theorem 5.3.1). This will be possible for $G_{\theta} / H_{\theta}$, in contrast to a reductive symmetric space $G / H$ for which such statement is still conjectural.

Here are the correspondences between $G / H$ and $G_{\theta} / H_{\theta}$ : 


$$
G / H \quad G_{\theta} / H_{\theta}
$$

Conjecture 3.3.10 Theorem 5.3.1: $\quad$ Reduction to connected case.

Conjecture 2.5.1 Theorem 1.1.6: $\quad$ Space form problem.

5.3. Reduction to connected subgroups. Hereafter, we shall denote a Cartan motion group by $G$ instead of $G_{\theta}$. The main result of this subsection is Theorem 5.3.1. We also reformulate this result into Theorem 5.3.2 under the assumption that $H$ is connected and $\bar{\theta}$-stable, which will then play a key role in our solution to the tangential version of the space form problem (see Section 5.5).

We start with a general case where $H$ is not necessarily $\bar{\theta}$-stable.

Theorem 5.3.1. Let $G$ be a Cartan motion group and $H$ a closed subgroup of $G$. Then the following two conditions are equivalent:

(i) A homogeneous space $G / H$ admits compact Clifford-Klein forms.

(ii) There exists a $\bar{\theta}$-stable connected subgroup $L$ satisfying the following two conditions (a) and (b).

(5.3.1)(a) $L \pitchfork H$ in $G$.

(5.3.1)(b) $L \backslash G / H$ is compact.

Note that the implication "(ii) $\Rightarrow$ (i)" is a 'tangential' analog of Theorem 3.3.1, whereas the implication "(i) $\Rightarrow$ (ii)" is a 'tangential' solution to Conjecture 3.3.10 for a homogeneous space of reductive type. The proof of Theorem 5.3.1 will be given in Section 5.4.

As a second formulation of Theorem 5.3.1, we shall reformulate it in a more explicit way for $\bar{\theta}$-stable $H$. To do this, we introduce a tangential analog of $\mathfrak{a}(L)$ given in (3.2.1) as follows: Let $G=K \ltimes \mathfrak{p}$ be a Cartan motion group. Recall from Section 3.2 that $\mathfrak{a}$ was taken as a maximal abelian subspace of $\mathfrak{p}$ (where, we remember the original reductive Lie algebra $\mathfrak{k}+\mathfrak{p}$ ). Then, for a subset $L$ in the Cartan motion group $G=K \ltimes \mathfrak{p}$, we define

$$
\mathfrak{a}(L):=K L K \cap \mathfrak{a} .
$$

Then Theorem 5.3.1 amounts to:

Theorem 5.3.2. Let $G$ be a Cartan motion group, and let $H$ be a $\bar{\theta}$-stable subgroup with at most finitely many connected components. Then, the following two conditions are equivalent:

(i) A homogeneous space $G / H$ admits compact Clifford-Klein forms.

(ii) There exists a subspace $W$ in $\mathfrak{p}$ satisfying the following two conditions (a) and (b).

$(5.3 .2)(\mathrm{a}) \mathfrak{a}(W) \cap \mathfrak{a}(H)=\{0\}$.

(5.3.2)(b) $\operatorname{dim}(W)+d(H)=d(G)$. 
We prove Theorem 5.3.2 by admitting Theorem 5.3.1. First, we prepare some necessary lemmas for this.

Lemma 5.3.3. Let $G=K \ltimes \mathfrak{p}$ be a Cartan motion group, and $H$ a $\bar{\theta}$-stable connected subgroup. Then:

1) $H=(K \cap H) \ltimes(\mathfrak{p} \cap H)$.

2) $\mathfrak{p} \cap H$ is a subspace of $\mathfrak{p}$.

3) $H$ has always a uniform lattice.

4) $H \sim_{s} \mathfrak{p} \cap H$.

Proof. 1) We note that $\exp : \operatorname{Lie}(\mathfrak{p}) \rightarrow \mathfrak{p}$ is the identity map if we identify $\operatorname{Lie}(\mathfrak{p})$ with $\mathfrak{p}$ as usual. Let $H^{\prime}$ be the image of $(K \cap H) \times(\operatorname{Lie}(\mathfrak{p}) \cap \mathfrak{h})$ by the following diffeomorphism:

$$
\phi:=\mathrm{id} \times \exp : K \times \operatorname{Lie}(\mathfrak{p}) \rightarrow K \ltimes \mathfrak{p}, \quad(k, X) \mapsto(k, X) .
$$

Clearly, $H^{\prime}$ is closed. We claim that $H^{\prime}$ is an open submanifold of $H$. This is because the Lie algebra $\mathfrak{h}$ of $H$ decomposes $\mathfrak{h}=(\mathfrak{k} \cap \mathfrak{h})+(\operatorname{Lie}(\mathfrak{p}) \cap \mathfrak{h})$, so that $\operatorname{dim} H^{\prime}=\operatorname{dim}(K \cap H)+\operatorname{dim}(\operatorname{Lie}(\mathfrak{p}) \cap \mathfrak{h})=\operatorname{dim} H$. Thus $H^{\prime}=H$ since $H$ is connected.

2) Since $\phi$ surjects $H$, we have $\mathfrak{p} \cap H \simeq \operatorname{Lie}(\mathfrak{p}) \cap \mathfrak{h}$ via $\phi$. Therefore, $\mathfrak{p} \cap H$ is a subspace of $\mathfrak{p}$.

3) A lattice of the vector space $\mathfrak{p} \cap H$ becomes a uniform lattice of $H$.

4) This is an immediate consequence of (1).

Remark 5.3.4. By the same argument, one sees easily that Lemma 5.3.3 still holds if the number of connected components of $H$ is finite.

The following lemma is a 'tangential' analog of Lemma 3.3.4. As in the proof of [Ko89a, Theorem 4.7], one could prove it by using the cohomological dimension of abstract groups. However, we shall supply much elementary approach for our Cartan motion groups below:

Lemma 5.3.5. Let $G$ be a Cartan motion group. Suppose that $L$ and $H$ are $\bar{\theta}$-stable subgroups with at most finitely many connected components. Assume $L \pitchfork H$. Then, we have:

1) $d(L)+d(H) \leq d(G)$.

2) $d(L)+d(H)=d(G)$ if and only if $L \backslash G / H$ is compact.

Proof. Let $\mathfrak{p}_{L}:=L \cap \mathfrak{p}$ and $\mathfrak{p}_{H}:=H \cap \mathfrak{p}$, then we have $d(L)=\operatorname{dim} \mathfrak{p}_{L}$ and $d(H)=\operatorname{dim} \mathfrak{p}_{H}$.

1) Since $L \sim \mathfrak{p}_{L}$ and $H \sim \mathfrak{p}_{H}$, we have $\mathfrak{p}_{L} \pitchfork \mathfrak{p}_{H}$. Then it follows from Lemma 3.1.7 that we have $\mathfrak{p}_{L} \cap \mathfrak{p}_{H}=\{0\}$, and thus $\operatorname{dim} \mathfrak{p}_{L}+\operatorname{dim} \mathfrak{p}_{H} \leq \mathfrak{p}$. 
2) First, assuming $d(L)+d(H)=d(G)$, we shall prove $G=L K H$. To see this, we note $\mathfrak{p}_{H} \sim \operatorname{Ad}(k) \mathfrak{p}_{H}$ for any $k \in K$. Therefore, we have $\mathfrak{p}_{L} \pitchfork \operatorname{Ad}(k) \mathfrak{p}_{H}$, and then $\mathfrak{p}_{L} \cap \operatorname{Ad}(k) \mathfrak{p}_{H}=\{0\}$. Thus we obtain $\mathfrak{p}_{L}+\operatorname{Ad}(k) \mathfrak{p}_{H}=\mathfrak{p}$ in light of dimension. Now, for any $(k, X) \in G$, we find $(e, Y) \in \mathfrak{p}_{L}$ and $(e, Z) \in \mathfrak{p}_{H}$ such that $X=Y+\operatorname{Ad}(k) Z$. This implies

$$
(k, X)=(e, Y)(k, 0)(e, Z) \in \mathfrak{p}_{L} K \mathfrak{p}_{H} \subset L K H .
$$

Hence, $G=\mathfrak{p}_{L} K \mathfrak{p}_{H}=L K H$. We have thus proved that $L \backslash G / H$ is compact.

Conversely, let us prove $d(L)+d(H)=d(G)$ if $L \backslash G / H$ is compact. It follows from Lemma 3.1.10 that $\mathfrak{p}_{L} \backslash G / \mathfrak{p}_{H}$ is compact. Now, $\mathfrak{p}$ is a closed subgroup containing $\mathfrak{p}_{L}$ and $\mathfrak{p}_{H}$. Therefore, $\mathfrak{p}_{L} \backslash \mathfrak{p} / \mathfrak{p}_{H}$ is a closed subset of the compact set $\mathfrak{p}_{L} \backslash G / \mathfrak{p}_{H}$. Since $\mathfrak{p}$ is a vector space, $\mathfrak{p}_{L} \backslash \mathfrak{p} / \mathfrak{p}_{H}$ is compact only if $\operatorname{dim} \mathfrak{p}_{L}+\operatorname{dim} \mathfrak{p}_{H} \geq$ $\operatorname{dim} \mathfrak{p}$. Hence, Lemma has been proved.

Next, we state a criterion of $\pitchfork$ and $\sim$ for Cartan motion groups (see Lemma 3.2.1 for the counterpart in the reductive case).

Lemma 5.3.6 ([Y05]). For any subsets $L$ and $H$ in $G$, we have

1) $L \pitchfork H$ in $G \Longleftrightarrow \mathfrak{a}(L) \pitchfork \mathfrak{a}(H)$ in $\mathfrak{a}$.

2) $L \sim H$ in $G \Longleftrightarrow \mathfrak{a}(L) \sim \mathfrak{a}(H)$ in $\mathfrak{a}$.

Here we recall (5.3.2) for the definition of $\mathfrak{a}(L)$.

Remark 5.3.7. Similar results hold for an arbitrary semidirect product group $G=K \ltimes_{\tau} V$ associated to a finite dimensional representation $(\tau, V)$ of a compact group $K$, if we replace $\mathfrak{a}$ by any subspace $V^{\prime}$ such that $\tau(K) V^{\prime}=V$.

Lemma 5.3.8. Let $G$ be a Cartan motion group, and $H$ its $\bar{\theta}$-stable subgroup with finitely many connected components. Then, $\mathfrak{a}(H)$ is a closed cone.

Proof. In light of $H=(K \cap H) \ltimes(\mathfrak{p} \cap H)$, Lemma follows from $\mathfrak{a}(H)=\mathfrak{a} \cap(K(\mathfrak{p} \cap$ $H) K)=\mathfrak{a} \cap \bigcup_{k \in K} \operatorname{Ad}(k)(\mathfrak{p} \cap H)$.

Finally, we prove Theorem 5.3.2.

Proof of Theorem 5.3.2. It is enough to prove the equivalence (5.3.1)(ii) $\Longleftrightarrow$ (5.3.2)(ii). For this, we shall set $W:=L \cap \mathfrak{p}$ when we start from (5.3.1), while we shall just set $L:=W$ when we start from (5.3.2). Then the following conditions are equivalent:

$$
\begin{aligned}
L \pitchfork H \text { in } G & \Longleftrightarrow W \pitchfork H \text { in } G & & \text { by Lemma } 5.3 .3(4) \\
& \Longleftrightarrow \mathfrak{a}(W) \pitchfork \mathfrak{a}(H) \text { in } \mathfrak{a} & & \text { by Lemma } 5.3 .6 \\
& \Longleftrightarrow \mathfrak{a}(W) \cap \mathfrak{a}(H)=\{0\} & & \text { by Lemmas } 3.1 .7 \text { and 5.3.8. }
\end{aligned}
$$


On the other hand, we also have:

$$
\begin{aligned}
L \backslash G / H \text { is compact } & \Longleftrightarrow W \backslash G / H \text { is compact } & \text { by Lemmas 3.1.10 and 5.3.3 } \\
& \Longleftrightarrow \operatorname{dim}(W)+d(H)=d(G) & \text { by Lemma 5.3.5. }
\end{aligned}
$$

Thus, the conditions (5.3.1)(ii) and (5.3.2)(ii) are equivalent.

Thus we have proved Theorem 5.3.2 by admitting Theorem 5.3.1, which we shall prove in the next subsection.

5.4. Proof of Theorem 5.3.1. This subsection gives a proof of Theorem 5.3.1. The implication (ii) $\Rightarrow$ (i) of Theorem 5.3.1 is an immediate consequence of Theorem 3.3.1 and Lemma 5.3.3(3).

On the other hand, the implication (i) $\Rightarrow$ (ii) follows from the next lemma.

Lemma 5.4.1. Suppose that $(\tau, V)$ is a finite dimensional real representation of a compact group $K$. We form a semidirect group $G=K \ltimes_{\tau} V$. For any discrete subgroup $\Gamma$ in $G$, there exists a subspace $W \subset V$ such that $\Gamma \sim_{s} W$ in $G$.

In fact, suppose $\Gamma$ is a cocompact discontinuous group for $G / H$. Let $W$ be the subspace of $\mathfrak{p}$ as in Lemma 5.4.1, and we set $L=W$. Then $L$ is a connected $\bar{\theta}$-stable subgroup satisfying (5.3.1)(a) and (b) by Lemma 3.1.9. Hence, Theorem 5.3.1 has been reduced to Lemma 5.4.1.

We need a generalization of results of Auslander [Au60], [Au61] and Wang [Wa63], as follows:

Lemma 5.4.2 ([Rg72, Theorem 8.24]). Let $G$ be a Lie group and $V$ a closed connected solvable normal subgroup. Let $\pi: G \rightarrow G / V$ be the natural quotient map. Given a closed subgroup $\Gamma$ of $G$, we write $K_{\Gamma}:=\overline{\pi(\Gamma)}$ for the closure of $\pi(\Gamma)$. If the identity component $\Gamma_{0}$ of $\Gamma$ is solvable, so is $\left(K_{\Gamma}\right)_{0}$.

Then, Lemma 5.4.1 is a consequence of the following Lemma.

Lemma 5.4.3. Suppose $G=K \ltimes_{\tau} V$ is as in Lemma 5.4.1. We write $\pi: G \rightarrow$ $G / V \simeq K$ for the natural quotient map. Suppose $\Gamma$ is a closed subgroup of $G$. Let $\left(K_{\Gamma}\right)_{0}$ be the subgroup of $K$ as in Lemma 5.4.2. If $\left(K_{\Gamma}\right)_{0}$ is abelian, then there exists a subspace $W \subset V$ such that $\Gamma \sim_{s} W$ in $G$.

Let us give a proof of Lemma 5.4.1 by admitting Lemma 5.4.3.

Proof of Lemma 5.4.1. Apply Lemma 5.4.2 to the case: $\Gamma$ is a discrete subgroup of a Cartan motion group $G=K \ltimes \mathfrak{p}$. Since $\Gamma_{0}=\{e\}$ is solvable, so is $\left(K_{\Gamma}\right)_{0}$. Then $\left(K_{\Gamma}\right)_{0}$ must be abelian because $\left(K_{\Gamma}\right)_{0}$ is compact group. Now, Lemma 5.4.1 follows from Lemma 5.4.3. 
The rest of this subsection is devoted to the proof of Lemma 5.4.3. In the course of the proof, we need to treat also the case where $\Gamma$ is not discrete.

To see Lemma 5.4.3, we start with the following multiplicative version of the Jordan decomposition. Let $K$ be a closed subgroup of $O(n)$ and $V=\mathbb{R}^{n}$. We form a semidirect product group $G:=K \ltimes V(\subset G L(n+1, \mathbb{R}))$. We define a subset $\widetilde{K}$ of $G$ consisting of elliptic elements as follows:

$$
\begin{aligned}
\widetilde{K} & :=\bigcup_{g \in V} g K g^{-1} \\
& =\left\{s \in G:\left\{s^{k}: k \in \mathbb{Z}\right\} \text { is relatively compact }\right\} .
\end{aligned}
$$

Lemma 5.4.4. 1) For any $g \in G$, there exist uniquely $s \in \widetilde{K}$ and $w \in V$ satisfying $g=s w=w s$.

2) Both $s$ and $w$ can be written as polynomials of $g$ in $M(n+1, \mathbb{R})$.

Proof. 1) We fix $g=(k, v) \in G$ once and for all. Let $V_{1} \subset V$ be the eigenspace of $k$ with eigenvalue 1 , and $V_{2}$ the orthogonal complement of $V_{1}$ in $V$. According to the direct sum decomposition $V=V_{1} \oplus V_{2}$, we write $v=v_{1}+v_{2}$. We set

$$
s:=\left(k, v_{2}\right), \quad w:=\left(e, v_{1}\right) .
$$

First, we claim $s \in \widetilde{K}$. Since the linear map (id $-k$ ) preserves $V_{2}$ and acts as an invertible transformation, we can take $u \in V_{2}$ such that $v_{2}=(\mathrm{id}-k) u$. Then, we obtain $s=\left(k, v_{2}\right)=(e, u)(k, 0)(e,-u) \in \widetilde{K}$.

Next, we verify $g=s w=w s$. In view of $k v_{1}=v_{1}$, we have

$$
\begin{aligned}
& w s=\left(e, v_{1}\right)\left(k, v_{2}\right)=\left(k, v_{1}+v_{2}\right)=g, \\
& s w=\left(k, v_{2}\right)\left(e, v_{1}\right)=\left(k, v_{2}+v_{1}\right)=g .
\end{aligned}
$$

Finally, the uniqueness follows immediately from $\widetilde{K} \cap V=\{(e, 0)\}$. Hence (1) is proved.

2) Admitting the existence of a polynomial $p(x)=\sum_{n=0}^{N} c_{n} x^{n}$ satisfying

$$
p(k)=0, \quad p(1)=0, \quad \text { and } \quad p^{\prime}(1)=1,
$$

we first finish the proof of $(2)$. For $g=(k, v)=\left(k, v_{1}+v_{2}\right)=\left(k,(\right.$ id $\left.-k) u+v_{1}\right)$, we have inductively,

$$
g^{n}=\left(k^{n},\left(\mathrm{id}-k^{n}\right) u+n v_{1}\right) .
$$

Therefore, we have

$$
\begin{aligned}
p(g) & =\sum_{n=0}^{N}\left(c_{n} k^{n},\left(c_{n} \mathrm{id}-c_{n} k^{n}\right) u+n c_{n} v_{1}\right) \\
& =\left(p(k),(p(1) \mathrm{id}-p(k)) u+p^{\prime}(1) v_{1}\right) \\
& =\left(0, v_{1}\right) .
\end{aligned}
$$


Thus, $s$ and $w$ are polynomials of $g$ given by:

$$
s=g-p(g), \quad w=p(g)+\mathrm{id} .
$$

Finally, let us construct a polynomial $p(x)$ satisfying (5.4.1). Suppose $q(x)$ is the minimal polynomial of the matrix

$$
\left(\begin{array}{c}
k \\
1
\end{array}\right) \in O(n+1)
$$

Clearly, $q(k)=0$ and $q(1)=0$. Since any element of $O(n+1)$ is semisimple, $q(x)$ has no multiple root. Hence $q^{\prime}(1) \neq 0$. Then $p(x):=\frac{q(x)}{q^{\prime}(1)}$ is the desired one. We have thus proved the lemma.

We are ready to prove Lemma 5.4.3. Without loss of generality, we may and do assume that $K$ is a closed subgroup of $O(V)$. Then, we can regard $G=K \ltimes V$ as a subgroup of $G L(n+1, \mathbb{R})$, where $n=\operatorname{dim} V$.

Proof of Lemma 5.4.3. We shall divide the proof into three steps.

Step 1: $\Gamma$ is abelian.

Assume that $\Gamma$ itself is abelian. The multiplicative Jordan decomposition $\gamma=$ $s w$ (see Lemma 5.4.4) for $\gamma \in \Gamma$ defines the projections:

$$
\begin{array}{ll}
p_{1}: \Gamma \longrightarrow G, & \gamma \longmapsto s, \\
p_{2}: \Gamma \longrightarrow V, & \gamma \longmapsto w .
\end{array}
$$

Under the assumption that $\Gamma$ is abelian, both $p_{1}$ and $p_{2}$ are group homomorphisms. In fact, if $\gamma_{i}=s_{i} w_{i}(i=1,2)$, then $\gamma_{1}, \gamma_{2}, s_{1}, s_{2}, w_{1}$ and $w_{2}$ mutually commute because $s_{i}$ and $w_{i}$ are polynomials of $\gamma_{i}$. We set

$$
S:=\overline{p_{1}(\Gamma)}, \quad W^{\prime}:=p_{2}(\Gamma)
$$

Then we claim $\Gamma \sim_{s} W^{\prime}$ in $G$. To see this, it is enough to show $S$ is compact because

$$
\Gamma \subset W^{\prime} S, \quad W^{\prime} \subset \Gamma S
$$

Since any element of $S$ commutes with each other, we can find a matrix $p \in$ $G L(n+1, \mathbb{R})$ such that $p s p^{-1}$ is simultaneously a diagonal matrix for all $s \in S$. As all the eigenvalues of $s$ have modulus one, we have $p S p^{-1} \subset\left\{\operatorname{diag}\left(\lambda_{1}, \ldots, \lambda_{n+1}\right)\right.$ : $\left.\left|\lambda_{i}\right|=1\right\}$, and thus $S$ is compact. We have thus proved $\Gamma \sim_{s} W^{\prime}$ in $G$.

On the other hand, let $W:=\operatorname{Span}_{\mathbb{R}} W^{\prime}$, then we have $W^{\prime} \sim_{s} W$ in $G$ by Lemma 3.1.9. Thus we have proved $\Gamma \sim_{s} W$ in $G$.

Step 2: $\pi(\Gamma)$ is abelian. 
Assume that $\pi(\Gamma)$ is abelian. We recall $K_{\Gamma}=\overline{\pi(\Gamma)}$, and we set $G_{\Gamma}:=K_{\Gamma} \ltimes V$, and $V_{\Gamma}:=\operatorname{Span}_{\mathbb{R}}(\Gamma \cap V)$. Our idea here is to remove the 'non-commutative part' of $\Gamma$ by taking a quotient map

$$
\varpi: G_{\Gamma} \rightarrow G_{\Gamma} / V_{\Gamma}
$$

This enables us to apply Step 1 to $G_{\Gamma} / V_{\Gamma}$.

First, let us verify that $V_{\Gamma}$ is a normal subgroup in $G_{\Gamma}\left(=K_{\Gamma} \ltimes V\right)$. It is enough to show that $V_{\Gamma}$ is $\pi(\Gamma)$-invariant. For any $v \in V$ and any $\gamma \in \Gamma$, we have

$$
\pi(\gamma) v=\gamma v \gamma^{-1}
$$

Here, $\pi(\gamma) v$ in the left-hand side is defined by the action of $K$ on $V$, while $\gamma v \gamma^{-1}$ in the right-hand side is the group multiplication in $G$. Thus, the action of $\pi(\Gamma)$ on $V$ preserves $\Gamma \cap V$, and then preserves also $V_{\Gamma}=\operatorname{Span}_{\mathbb{R}}(\Gamma \cap V)$.

Next, let us verify $\varpi(\Gamma)$ is abelian. The natural quotient map $G_{\Gamma} / V_{\Gamma} \rightarrow G_{\Gamma} / V$ induces a surjective homomorphism $\mu: \varpi(\Gamma) \rightarrow \pi(\Gamma)$. Then $\mu$ is also injective because $V \cap \Gamma \subset V_{\Gamma}$. Hence $\varpi(\Gamma)$ is abelian.

Applying Step 1 to $G_{\Gamma} / V_{\Gamma}\left(\simeq K_{\Gamma} \ltimes V / V_{\Gamma}\right)$ and its abelian subgroup $\varpi(\Gamma)$, we find a subspace $W^{\prime} \subset V / V_{\Gamma}$ such that

$$
\varpi(\Gamma) \sim_{s} W^{\prime} \text { in } G_{\Gamma} / V_{\Gamma} .
$$

It follows from Lemma 3.1.12 that $\varpi^{-1}(\varpi(\Gamma)) \sim_{s} \varpi^{-1}\left(W^{\prime}\right)$ in $G_{\Gamma}$. We set $W:=$ $\varpi^{-1}\left(W^{\prime}\right)$. Since $G_{\Gamma} \subset G$, we have

$$
\varpi^{-1}(\varpi(\Gamma)) \sim_{s} W \text { in } G .
$$

To see $\Gamma \sim_{s} W$ in $G$, it is enough to show $\Gamma \sim_{s} \varpi^{-1}(\varpi(\Gamma))$ in $G$, which will follow if we prove $\varpi^{-1}(\varpi(\Gamma)) / \Gamma$ is compact by Lemma 3.1.9. This then follows from

$$
\varpi^{-1}(\varpi(\Gamma)) / \Gamma=\Gamma V_{\Gamma} / \Gamma=V_{\Gamma} / V_{\Gamma} \cap \Gamma=V_{\Gamma} / V \cap \Gamma
$$

because $V_{\Gamma}=\operatorname{Span}_{\mathbb{R}}(V \cap \Gamma)$ contains $V \cap \Gamma$ as a cocompact subgroup. Here, the first two equalities in (5.4.4) are clear, and the last one follows from $V_{\Gamma} \cap \Gamma=V \cap \Gamma$, as is seen by taking the intersection of $\Gamma$ with $V \cap \Gamma \subset V_{\Gamma} \subset V$. Hence, we have proved $\Gamma \sim_{s} W$ in $G$.

Step 3: $\left(K_{\Gamma}\right)_{0}$ is abelian.

Finally, suppose $\Gamma$ satisfies the assumption of Lemma, namely, $\left(K_{\Gamma}\right)_{0}$ is abelian. We set a subgroup $\Gamma_{(0)}:=\pi^{-1}\left(\left(K_{\Gamma}\right)_{0}\right) \cap \Gamma$ of $\Gamma$. Since $\pi\left(\Gamma_{(0)}\right) \subset\left(K_{\Gamma}\right)_{0}, \pi\left(\Gamma_{(0)}\right)$ is abelian. Applying Step 2 to $\left(G, \Gamma_{(0)}\right)$, there exists a subspace $W \subset V$ such that

$$
\Gamma_{(0)} \sim_{s} W \text { in } G .
$$

To see $\Gamma \sim_{s} W$ in $G$, it is enough to prove $\Gamma / \Gamma_{(0)}$ is compact.

Since $K$ is compact, $K_{\Gamma} /\left(K_{\Gamma}\right)_{0}$ is a finite group. Then, the composition

$$
\bar{\pi}: \Gamma \rightarrow K_{\Gamma} \rightarrow K_{\Gamma} /\left(K_{\Gamma}\right)_{0}
$$


is a surjective homomorphism, because $\pi(\Gamma)$ is dense in $K_{\Gamma}$. On the other hand, we have $\operatorname{Ker}(\bar{\pi})=\Gamma_{(0)}$. Thus, $\bar{\pi}$ induces the isomorphism:

$$
\Gamma / \Gamma_{(0)} \simeq K_{\Gamma} /\left(K_{\Gamma}\right)_{0} .
$$

Thus, $\Gamma / \Gamma_{(0)}$ is finite. Hence, $\Gamma \sim_{s} W$ in $G$. This completes the proof of Lemma 5.4.3.

Thus the proof of Theorem 5.3.1 is completed.

Remark 5.4.5. We did not assume $\Gamma$ is discrete in Lemma 5.4.3. But, if $\Gamma$ is a discrete subgroup, then $\left(K_{\Gamma}\right)_{0}$ is abelian by Lemma 5.4 .2 , and therefore, the semidirect product group $\left(G_{\Gamma}\right)_{0}:=\left(K_{\Gamma}\right)_{0} \ltimes V$ is solvable. Thus, $\Gamma$ is virtually solvable, as $\Gamma_{(0)}$ is contained in $\left(G_{\Gamma}\right)_{0}$ and $\Gamma_{(0)}$ is of finite index in $\Gamma$. Then, it follows from Fried and Goldman [FG83, Theorem 1.4] that there exists a closed subgroup $L$ of $\left(G_{\Gamma}\right)_{0}$ such that $\Gamma_{(0)}$ is cocompact in $L$ and $L$ is of finitely many components.

5.5. Solution to tangential space form problem. So far, we have discussed a general theory concerning Problem A for tangential symmetric space associated to reductive symmetric spaces. Building on it, we shall study specific case, namely, the tangential space form problem.

This subsection gives an outline of the proof of Theorem 1.1.6. The proof will be completed in Section 5.7.

Theorem 5.3.1 has reduced the existence problem of a cocompact discontinuous group $\Gamma$ for the tangential symmetric space $G / H$ to that of a certain connected group $L$. However, we shall find that the latter problem involves surprisingly rich ingredients even for a fairly simple pair $(G, H)$. Theorem 1.1.6 treats the case where $G / H$ (previously denoted by $G_{\theta} / H_{\theta}$ ) is the tangential symmetric space of the symmetric space $O(p+1, q) / O(p, q)$, and classifies the pair $(p, q)$ of integers for which such $L$ exists. Our argument relies on Adams' theorem on vector fields on spheres $([$ Ad62] $)$. Our strategy of proof and related classical results are listed in the following proposition.

Proposition 5.5.1. The following seven conditions on the pair $(p, q)$ of natural numbers are equivalent:

i) The tangential symmetric space of $O(p+1, q) / O(p, q)$ admits a compact Clifford-Klein form.

ii) There exists a bilinear map $f: \mathbb{R}^{p+1} \times \mathbb{R}^{q} \rightarrow \mathbb{R}^{q}$ such that

$$
f(v, w)=0 \text { only if } v=0 \text { or } w=0 .
$$

iii) There exists a bilinear map $f: \mathbb{R}^{p+1} \times \mathbb{R}^{q} \rightarrow \mathbb{R}^{q}$ such that

$$
\|v\| \cdot\|w\|=\|f(v, w)\| \quad \text { for any } v \in \mathbb{R}^{p+1} \text { and } w \in \mathbb{R}^{q} .
$$


iv) There exists an injective linear map $F: \mathbb{R}^{p} \rightarrow M(q, \mathbb{R})$ such that Image $F \subset$ $\mathfrak{o}(q) \cap \mathbb{R} \cdot O(q)$.

v) There is an identity $\left(x_{1}^{2}+\cdots+x_{p+1}^{2}\right) \cdot\left(y_{1}^{2}+\cdots+y_{q}^{2}\right)=z_{1}^{2}+\cdots+z_{q}^{2}$ such that $z_{1}, \ldots, z_{q}$ are bilinear functions of $x_{1}, \ldots, x_{p+1}$ and $y_{1}, \ldots, y_{q}$.

vi) There exist $p$ vector fields on the sphere $S^{q-1}$ which are linearly independent at every point.

vii) $p<\rho(q) \quad($ see $(2.6 .1))$.

Theorem 1.1.6 corresponds to the equivalence of (i) and (vii) in Proposition.

Remark 5.5.2. We point out that there is a mysterious duality between $p$ and $q$ in (i) and (vi). That is, in (i), the tangential symmetric space of $O(p+1, q) / O(p, q)$ is diffeomorphic to

the trivial $\mathbb{R}^{q}$-bundle over $S^{p}$

as $K$-equivariant fiber bundles, whereas (vi) defines

$$
\text { the trivial } \mathbb{R}^{p} \text {-bundle over } S^{q-1}
$$

as a subbundle of the tangent bundle $T\left(S^{q-1}\right)$ of $S^{q-1}$.

The proof of Proposition will be organized as follows:

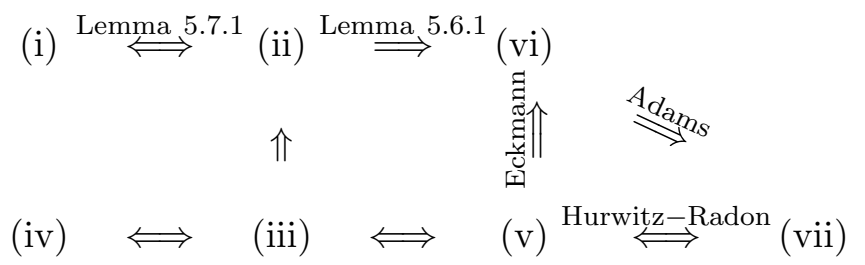

(v) $\Longleftrightarrow$ (vii): This equivalence was established by Hurwitz [Hu23] and Radon [Rd22]. See also Eckmann [E42], [E94].

(v) $\Longrightarrow$ (vi): This statement was proved in Eckmann [E42].

(vi) $\Longrightarrow$ (vii): This statement was proved in Adams [Ad62].

(iii) $\Longrightarrow$ (ii): Obvious.

(iii) $\Longleftrightarrow(\mathrm{v})$ : Obvious.

(iii) $\Longleftrightarrow$ (iv): This equivalence is an easy observation of linear algebra by the correspondence $f((x, c), \cdot)=F(x)+c I_{q}$ for $(x, c) \in \mathbb{R}^{p} \oplus \mathbb{R}$.

(ii) $\Longrightarrow$ (vi): This proof is in the same line of the argument $(\mathrm{v}) \Longrightarrow(\mathrm{vi})$, but we shall give a proof in Lemma 5.6.1 for the sake of completeness.

Therefore, the proof of Proposition will be completed if we show the equivalence (i) $\Longleftrightarrow$ (ii). This will be carried out in Lemma 5.7.1. 
We shall also give an alternative proof of the implication (vii) $\Longrightarrow$ (iii) (or (vii) $\Longrightarrow(\mathrm{v})$ ) in Lemma 5.6.2 as an application of the theory of Clifford algebras associated to indefinite quadratic forms.

5.6. Results of Hurwitz-Radon-Eckmann. This subsection provides a simple and self-contained proof of the implication (ii) $\Rightarrow(v i)$ (see Lemma 5.6.1) and (vii) $\Rightarrow$ (iii) (see Lemma 5.6.2).

Lemma 5.6.1. Let $(p, q)$ be a pair of natural numbers, and assume that there exists a bilinear map $f: \mathbb{R}^{p+1} \times \mathbb{R}^{q} \rightarrow \mathbb{R}^{q}$ satisfying (5.7.3). Then, there exists $p$ vector fields on the sphere $S^{q-1}$ which are linearly independent at every point.

Proof. Let $\left\{e_{0}, \ldots, e_{p}\right\}$ be the standard basis of $\mathbb{R}^{p+1}$. Since $f(\cdot, w): \mathbb{R}^{p+1} \rightarrow \mathbb{R}^{q}$ is injective for any $w \in S^{q-1}, f\left(e_{0}, w\right), \ldots, f\left(e_{p}, w\right) \in \mathbb{R}^{q}$ are linearly independent. In light that $g:=f\left(e_{0}, \cdot\right): \mathbb{R}^{q} \rightarrow \mathbb{R}^{q}$ is invertible, $t_{i}(w):=g^{-1} f\left(e_{i}, w\right)(0 \leq i \leq p)$ are well defined and linearly independent. We note $t_{0}(w)=w$. Now we set

$$
Z_{i}(w):=t_{i}(w)-\left\langle t_{i}(w), w\right\rangle w \quad(i=1, \ldots, p) .
$$

Then, $Z_{1}, \ldots, Z_{p}$ define $p$ vector fields on $S^{q-1}$ which are linearly independent at every point.

Lemma 5.6.2. For any positive integer $q$, there exists a linear map

$$
f: \mathbb{R}^{\rho(q)} \rightarrow M(q, \mathbb{R})
$$

such that ${ }^{t} f(v) f(v)=\|v\|^{2} I_{q}$ for any $v \in \mathbb{R}^{\rho(q)}$.

This Lemma is due to Hurwitz [Hu23] and Radon [Rd22]. Later, Eckmann [E42] also gave a simple proof (see also [E94]). Here, we give yet another simple proof based on the Clifford algebra associated to the indefinite quadratic form.

Proof. It is sufficient to prove Lemma in the case where $q$ is of the form $q=2^{k}$ because the Hurwitz-Radon number $\rho(q)$ remains the same if we multiply $q$ by any odd number. Henceforth, suppose $q=2^{4 \alpha+\beta}(\beta=0,1,2,3)$. According to the value $\beta$, we set

Case 1) $\quad \beta=0,1$ or $2 . \quad(r, s):=(\beta, 8 \alpha+\beta)$.

Case 2) $\quad \beta=3 . \quad(r, s):=(0,8 \alpha+6)$.

In either case, $r-s-1 \equiv \pm 1 \bmod 8$ and $q=2^{(r+s) / 2}$. Therefore, it follows from Proposition 4.4.1 that there exists an algebra isomorphism $\iota: C(r, s) \stackrel{\sim}{\rightarrow} M(q, \mathbb{R})$, and we have from Lemma 4.7.10

$$
{ }^{t} \iota(x)=\iota\left(x^{*}\right) .
$$

We assert: 
Claim: For $q=2^{4 \alpha+\beta}$, we set $p:=\rho(q)=8 \alpha+2^{\beta}$. Then, there exist $x_{1}, \ldots, x_{p-1} \in$ $C(r, s)$ with the following three conditions:

1) $x_{i}^{2}=-1 \quad(1 \leq i \leq p-1)$,

2) $x_{i} x_{j}+x_{j} x_{i}=0 \quad(i \neq j, 1 \leq i, j \leq p-1)$,

3) $x_{i}{ }^{*}=-x_{i} \quad(1 \leq i \leq p-1)$.

First, let us complete the proof of Lemma by admitting Claim. The substitution of (3) into (1) and (2) leads to

$$
x_{i}{ }^{*} x_{i}=1, \quad x_{i}{ }^{*} x_{j}+x_{j}{ }^{*} x_{i}=0 \quad(i \neq j),
$$

for $1 \leq i, j \leq p-1$. Clearly the relation (5.6.2) still holds for $0 \leq i, j \leq p-1$ if we set $x_{0}:=1$. We now define a linear map $f$ by

$$
f: \mathbb{R}^{p} \mapsto M(q, \mathbb{R}), \quad e_{i} \mapsto \iota\left(x_{i}\right) \quad(0 \leq i \leq p-1) .
$$

Then, if $v \in \mathbb{R}^{p}$ is written as $\sum_{i=0}^{p-1} c_{i} e_{i}$, we have:

$$
{ }^{t} f(v) \cdot f(v)=\left(\sum_{i=0}^{p-1} c_{i}{ }^{t} \iota\left(x_{i}\right)\right)\left(\sum_{i=0}^{p-1} c_{i} \iota\left(x_{i}\right)\right)=\sum_{i=0}^{p-1} c_{i}^{2} I_{q}=\|v\|^{2} I_{q} .
$$

Here, the second equality follows from (5.6.1) and (5.6.2). Therefore, Lemma 5.6.2 has been proved by assuming Claim.

Finally, let us prove Claim.

Case 1) $\beta=0$ or $1 . \quad x_{i}:=v_{i}^{-} \quad(i=1, \ldots, p-1)$.

Case 2$) \beta=2$ or $3 . \quad x_{i}:=v_{i}^{-} \quad(i=1, \ldots, p-2) ; \quad x_{p-1}:=J_{-}=v_{1}^{-} \ldots v_{p-1}^{-}$.

Then, an easy computation shows the conditions (1), (2) and (3) in Claim.

Hence, the proof of Lemma completed.

5.7. Proof of Theorem 1.1.6. This subsection completes the proof of Theorem 1.1.6 by showing the equivalence of the conditions (i) and (ii) in Proposition 5.5.1, namely, the following:

Lemma 5.7.1. Let $(p, q)$ be a pair of natural numbers. Then, the following two conditions are equivalent:

i) The tangential symmetric space of $O(p+1, q) / O(p, q)$ admits a compact Clifford-Klein form.

ii) There exists a bilinear map $f: \mathbb{R}^{p+1} \times \mathbb{R}^{q} \rightarrow \mathbb{R}^{q}$ such that

$$
f(v, w)=0 \text { only if } v=0 \text { or } w=0 .
$$


Throughout this subsection, suppose $G / H$ is the tangential symmetric space of $O(p+1, q) / O(p, q)$. Here we realize $O(p+1, q)$ in $G L(p+q+1, \mathbb{R})$ in the standard way (see (4.1.1)) and

$$
O(p, q)=\left\{g \in O(p+1, q): g e_{0}=e_{0}\right\},
$$

where $\left\{e_{0}, \ldots, e_{p}\right\}$ denotes the standard basis of the Euclidean space $\mathbb{R}^{p+q+1}$. We take $\theta$ to be the standard Cartan involution of $O(p+1, q)$, given by $\theta g={ }^{t} g^{-1}$ for $g \in O(p+1, q)$. Then, the maximal compact subgroup $K=O(p+1) \times O(q)$ acts on

$$
\mathfrak{p}=\left\{\left({ }_{X}{ }^{t} X\right) \mid X \in M(q, p+1 ; \mathbb{R})\right\} \simeq M(q, p+1 ; \mathbb{R}),
$$

by $\mathfrak{p} \rightarrow \mathfrak{p}, X \mapsto k_{2} X k_{1}^{-1} \quad\left(k_{1} \in O(p+1), k_{2} \in O(q)\right)$.

It follows from Theorem 5.3.2 that $G / H$ admits a compact Clifford-Klein form if and only if the following condition (i) ${ }^{\prime}$ holds. Thus, to show the equivalence (i) $\Longleftrightarrow$ (ii), it is sufficient to prove the equivalence (i) $)^{\prime} \Longleftrightarrow$ (ii)

i) $)^{\prime}$ There exists a $q$-dimensional subspace $W \subset \mathfrak{p}$ satisfying

$$
\mathfrak{a}(W) \cap \mathfrak{a}(H)=\{0\} .
$$

ii) There exists a bilinear map $f: \mathbb{R}^{p+1} \times \mathbb{R}^{q} \rightarrow \mathbb{R}^{q}$ such that

$$
f(v, w)=0 \text { only if } v=0 \text { or } w=0 .
$$

Proof. Given a $q$-dimensional subspace $W$ in $\mathfrak{p}$, we fix a linear isomorphism

$$
\tilde{f}: \mathbb{R}^{q} \stackrel{\sim}{\rightarrow} W \subset M(q, p+1 ; \mathbb{R}) \simeq \operatorname{Hom}\left(\mathbb{R}^{p+1}, \mathbb{R}^{q}\right),
$$

and then define a bilinear map

$$
f: \mathbb{R}^{p+1} \times \mathbb{R}^{q} \rightarrow \mathbb{R}^{q}
$$

by using the canonical identification

$$
\tilde{f} \leftrightarrow f, \quad \operatorname{Hom}\left(\mathbb{R}^{q}, \operatorname{Hom}\left(\mathbb{R}^{p+1}, \mathbb{R}^{q}\right)\right) \simeq \operatorname{Hom}\left(\mathbb{R}^{p+1} \otimes \mathbb{R}^{q}, \mathbb{R}^{q}\right) .
$$

Conversely, given a bilinear map $f$ satisfying (5.7.3), we define a subspace $W_{f}$ of $M(q, p+1 ; \mathbb{R})$ by

$$
W_{f}:=\text { Image } \tilde{f} \quad(\text { see } 5.7 .5) .
$$

Then, $\operatorname{dim} W_{f}=q$ because $\tilde{f}$ is injective. Now, the equivalence (i) $\Longleftrightarrow$ (ii) follows from the next claim.

Claim: The condition (5.7.2) and (5.7.3) are equivalent via the correspondence $f \leftrightarrow W=W_{f}$.

To see Claim, let us find $\mathfrak{a}(H)$. Via the identification (5.7.1), we have

$$
H \cap \mathfrak{p}=\left\{X \in \mathfrak{p}: X e_{0}=0\right\} .
$$


Then $\mathfrak{a}(H)$ (see (5.3.2)) is computed as follows:

$$
\begin{aligned}
\mathfrak{a}(H) & =K H K \cap \mathfrak{a} \\
& =\{X \in \mathfrak{a}:(e, X) \in K H K\} \\
& =\left\{X \in \mathfrak{a}: k_{1} X k_{2}^{-1} \in H \cap \mathfrak{p} \quad \text { for some }\left(k_{1}, k_{2}\right) \in K\right\} \\
& =\left\{X \in \mathfrak{a}: k_{1} X k_{2}^{-1} e_{0}=0 \quad \text { for some }\left(k_{1}, k_{2}\right) \in K\right\} \\
& =\left\{X \in \mathfrak{a}: X v=0 \text { for some non-zero } v \in \mathbb{R}^{p+1}\right\} .
\end{aligned}
$$

Suppose that (ii) fails. We shall show (i) fails. Take $v \in \mathbb{R}^{p+1} \backslash\{0\}$ and $w \in$ $\mathbb{R}^{q} \backslash\{0\}$ such that $f(v, w)=0$. We set $X:=\tilde{f}(w) \in W$. Then $X \neq 0$ and $X v=$ $f(v, w)=0$. In light of $W \subset K \mathfrak{a}(W) K$, we can find $k_{1}, k_{2} \in K$ and a non-zero $X^{\prime} \in \mathfrak{a}(W)$ such that $X=k_{1} X^{\prime} k_{2}$. Then $v^{\prime}:=k_{2} v \neq 0$ and $X^{\prime} v^{\prime}=k_{1}^{-1} X v=0$. Hence $X^{\prime} \in \mathfrak{a}(H)$ by (5.7.6). Therefore we have $X^{\prime} \in \mathfrak{a}(W) \cap \mathfrak{a}(H)$. Hence (i) fails. Likewise, we see if (i) fails then (ii) fails by using $\mathfrak{a}(W) \subset K W K$.

Now, the proof of Theorem 1.1.6 has been completed.

\section{REFERENCES}

[AmS97] H. Abels, G. A. Margulis And G. A. Soifer, Properly discontinuous groups of affine transformations with orthogonal linear part, C. R. Acad. Sci. Paris Ser. I Math. 324 (1997), 253-258.

[Ad62] J. F. Adams, Vector fields on spheres, Ann. of Math. 75 (1962), 603-632.

[ABS64] M. F. Atiyah, R. Bott And A. Shapiro, Clifford modules, Topology 3 (1964), 3-38.

[AtH61] M. F. ATIYAh And F. HiRzebruch, Bott periodicity and the parallelizability of the spheres, Proc. Cambridge Philos. Soc. 57 (1961), 223-226.

[Au60] L. Auslander, Bieberbach's theorem on space groups and discrete subgroups of Lie groups, Ann. of Math. 71 (1960), 579-590.

[Au61] L. Auslander, Bieberbach's theorem on space groups and discrete uniform subgroups of Lie groups, II, Amer. J. Math. 83 (1961), 276-280.

[Au64] L. Auslander, The structure of compact locally affine manifolds, Topology 3 (1964), 131-139.

[Bn96] Y. Benoist, Actions propres sur les espaces homogènes réductifs, Ann. of Math. 144 (1996), 315-347.

[BnL92] Y. Benoist And F. Labourie, Sur les espaces homogènes modèles de variétés compactes, I. H. E. S. Publ. Math. 76 (1992), 99-109.

[Br57] M. Berger, Les espaces symétriques non compacts, Ann. Sci. École Norm. Sup. 74 (1957), 85-177.

[Bo63] A. Borel, Compact Clifford-Klein forms of symmetric spaces, Topology 2 (1963), 111122.

[BoH62] A. Borel ANd HARISH-ChANDrA, Arithmetic subgroups of algebraic groups, Ann. of Math. (2), 75 (1962), 485-535.

[BoW00] A. Borel and N. Wallach, Continuous Cohomology, Discrete Subgroups, and Representations of Reductive Groups, 2nd edn., Math. Surveys and Monographs 67, Amer. Math. Soc. 2000. 
[BtM58] R. Bott And J. Milnor, On the parallelizability of the spheres, Bull. Amer. Math. Soc. 64 (1958), 87-89.

[CaM62] E. Calabi And L. Markus, Relativistic space forms, Ann. of Math. 75 (1962), 63-76.

[Co94] K. CoRlette, Harmonic maps, rigidity and Hodge theory, ICM-94, Proceedings (1994), 465-471.

[E42] B. Eckmann, Gruppentheoretischer Beweis des Satzes von Hurwitz-Radon über die Komposition quadratischer Formen, Comment. Math. Helv. 15 (1942/43), 358-366.

[E94] B. ECKMANN, Hurwitz-Radon matrices revisited: from effective solution of the Hurwitz matrix equations to Bott periodicity, The Hilton Symposium 1993 (Montreal, PQ), CRM Proc. Lecture Notes, 6, Amer. Math. Soc., Providence, RI, (1994), 23-35.

[FG83] D. FRIED AND W. Goldman, Three-dimensional affine crystallographic groups, Adv. Math. 47 (1983) 1-49.

[G85] W. Goldman, Nonstandard Lorentz space forms, J. Differential Geometry 21 (1985), 301-308.

[HoS41] H. Hopf AND H. SAmelson, Ein Satz über die Wirkungsräume geschlossener Liescher Gruppen, Comment. Math. Helv. 13 (1941), 240-251.

[Hu23] A. HuRwitz, Über die Komposition der quadratischen Formen, Math. Ann. 88 (1923), $1-25$.

[IW04] A. IOZZI AND D. WitTE, Tessellations of homogeneous space of classical group of real rank two, Geom. Dedicata 103 (2004), 115-191.

[KK85] S. KaneyUKi And M. KozaI, Paracomplex structures and affine symmetric space, Tokyo J. Math. 8 (1985), 81-98.

[Ke58] M. A. Kervaire, Non-parallelizability of the $n$-sphere for $n>7$, Proc. Nat. Acad. Sci., Wash. 44 (1958), 280-283.

[K196] B. KLINGLER, Complétude des variétés lorentziennes à courbure constante, Math. Ann. 306 (1996), 353-370.

[KN69] S. Kobayashi and K. Nomizu, Foundations of Differential Geometry. Vol. II, Interscience Publishers (1969).

[Ko88] T. Kobayashi, Properly discontinuous action on reductive homogeneous spaces, Seminar Reports of Unitary Representation (ed. H. Yamada) 8 (1988), 17-22, (in Japanese).

[Ko89a] T. Kobayashi, Proper action on a homogeneous space of reductive type, Math. Ann. 285 (1989), 249-263.

[Ko89b] T. KoBAyAshi, Homogeneous spaces with indefinite metric and discontinuous groups, Proceedings of the 36th Symposium on Differential Geometry, (1989), 104-116, (in Japanese).

[Ko90a] T. Kobayashi, Discontinuous group in a homogeneous space of reductive type, Seminar Reports of Unitary Representation (eds. T. Kawazoe et al.) 10 (1990), 41-45, the ICMsatellite conference at Fuji-Kawaguchiko.

[Ko90b] T. KobaYAShi, Discontinuous groups acting on non-Riemannian homogeneous spaces, RIMS Kokyuroku (ed. I. Satake), 737 (1990), 6-29.

[Ko92a] T. KoBAYASHI, Discontinuous groups acting on homogeneous spaces of reductive type, Representation Theory of Lie Groups and Lie Algebras at Fuji-Kawaguchiko, 1990 AugustSeptember, eds. T.Kawazoe, T.Oshima and S.Sano, World Scientific, 1992, 59-75.

[Ko92b] T. Kobayashi, A necessary condition for the existence of compact Clifford-Klein forms of homogeneous spaces of reductive type, Duke Math. J. 67 (1992), 653-664.

[Ko93] T. KoBAYASHI, On discontinuous groups acting on homogeneous spaces with noncompact isotropy subgroups, J. Geometry and Physics 12 (1993), 133-144.

[Ko94a] T. Kobayashi, Discrete decomposability of the restriction of $A_{\mathfrak{q}}(\lambda)$ with respect to reductive subgroups and its applications, Invent. Math. 117 (1994), 181-205. 
[Ko94b] T. Kobayashi, Compact Clifford-Klein forms and Clifford modules, unpublished note (1994).

[Ko96a] T. Kobayashi, Criterion of proper actions on homogeneous spaces of reductive groups, J. Lie Theory 6 (1996), 147-163.

[Ko96b] T. Kobayashi, Discontinuous groups and Clifford-Klein forms of pseudo-Riemannian homogeneous manifolds, In: Lecture Notes of the European School, August 1994, eds. H. Schlichtkrull and B. Ørsted, Perspectives in Math. 17. Academic Press (1996), 99-165.

[Ko98a] T. Kobayashi, Deformation of compact Clifford-Klein forms of indefinite-Riemannian homogeneous manifolds, Math. Ann. 310 (1998), 394-408.

[Ko98b] T. KoBAYASHI, Harmonic analysis on homogeneous manifolds of reductive type and unitary representation theory, Selected papers on harmonic analysis, groups, and invariants, Amer. Math. Soc. Transl. Ser. 2, 183 (1998), 1-31.

[Ko98c] T. Kobayashi, Discrete decomposability of the restriction of $A_{\mathfrak{q}}(\lambda)$ with respect to reductive subgroups $I I$ - micro-local analysis and asymptotic $K$-support, Ann. of Math. 147 (1998), 709-729.

[Ko01] T. KoBAYASHI, Discontinuous groups for non-Riemannian homogeneous spaces, Mathematics Unlimited-2001 and Beyond, (eds. B. Engquist and W. Schmid), Springer (2001), 723-747.

[Ko02] T. KoBAYASHI, Introduction to actions of discrete groups on pseudo-Riemannian homogeneous manifolds, Acta Appl. Math. 73 (2002), 115-131.

[Ko05] T. KobayAshi, Theory of discretely decomposable restrictions of unitary representations of semisimple Lie groups and some applications, Sugaku Expositions 18, Amer. Math. Soc. (2005), 1-37 (translated from Sugaku 51, Math. Soc. Japan (1999), 337-356, in Japanese).

[KoO90] T. Kobayashi And K. OnO, Note on Hirzebruch's proportionality principle, J. Fac. Univ. of Tokyo 37 (1990), 71-87.

[Ku81] R. S. Kulkarni, Proper actions and pseudo-Riemannian space forms, Adv. Math. 40 (1981), 10-51.

[L96] F. LABOURIE, Quelques résultats récents sur les espaces localement homogènes compacts, Symposia Mathematica (en l'honneur d'Eugenio Calabi) (1996), 267-283.

[LMZ95] F. Labourie, S. Mozes And R. J. Zimmer, On manifolds locally modeled on nonRiemannian homogeneous spaces, GAFA 5 (1995), 955-965.

[LZ95] F. LABOURIE AND R. J. ZimmeR, On the non-existence of cocompact lattices for $S L(n) / S L(m)$, Math. Res. Lett. 2 (1995), 75-77.

[Ma83] G. A. MARGULIS, Free completely discontinuous groups of affine transformations, Soviet Math. Dokl. 28 (1983), 435-439.

[Ma97] G. A. MARGULis, Existence of compact quotients of homogeneous spaces, measurably proper actions, and decay of matrix coefficients, Bull. Soc. Math. France 125 (1997), 447456.

[Ma00] G. A. Margulis, Problems and conjectures in rigidity theory, Mathematics: Frontiers and Perspectives, Amer. Math. Soc. (2000), 161-174.

[MoT62] G. D. Mostow And T. Tamagawa, On the compactness of arithmetically defined homogeneous spaces, Ann. of Math. 76 (1962), 446-463.

[O98] H. ОH, Tempered subgroups and representations with minimal decay of matrix coefficients, Bull. Soc. Math. France 126 (1998), 355-380.

[OW00] H. Оh And D. WitTe, New examples of compact Clifford-Klein forms of homogeneous spaces of $S O(2, n)$, Internat. Math. Res. Not. 5 (2000), 235-251.

[OW02] H. OH AND D. WitTE, Compact Clifford-Klein forms of homogeneous spaces of $S O(2, n)$, Geom. Dedicata 89 (2002), 25-57.

[P61] R. S. PALAIS, On the existence of slices for actions of noncompact Lie groups, Ann. of Math. 73 (1961), 295-323. 
[Rd22] J. RAdon, Lineare Scharen orthogonaler Matrizen, Abh. math. Sem. Hamburg 1 (1922), $1-14$.

[Rg72] M. Raghunathan, Discrete Subgroups of Lie Groups, Ergebnisse der Mathematik und ihrer Grenzgebiete, 68 (1972), Springer.

[Sa00] F. SAlEIn, Variété's anti-de Sitter de dimension 3 possédant un champ de Killing non trivial, Ann. Inst. Fourier 50 (2000), 257-284.

[Sel60] A. SELBERG, On discontinuous groups in higher-dimensional symmetric spaces, Contributions to functional theory, Bombay, (1960), 147-164.

[Ser71] J. P. SERre, Cohomologie des groupes discrètes, Ann. of Math. Studies 70 (1971), $77-169$.

[Sh00] Y. Shalom, Rigidity, unitary representations of semisimple groups, and fundamental groups of manifolds with rank one transformation group, Ann. of Math. 152 (2000), 113182.

[Wa63] H. C. WANG, On the deformations of lattices in a Lie groups, Amer. J. Math. 85 (1963), $189-212$

[Wo62] J. A. Wolf, The Clifford-Klein space forms of indefinite metric, Ann. of Math. $\mathbf{7 5}$ (1962), 77-80.

[Wo64] J. A. Wolf, Isotropic manifolds of indefinite metric, Comment. Math. Helv. 36 (1964), 21-64.

[Wo84] J. A. Wolf, Spaces of Constant Curvature, 5th edn., Publish of Perish, Inc., Wilmington, Delaware, 1984.

[Y05] T. Yoshino, Criterion of proper discontinuity in a Cartan motion group, submitted to Int. J. of Math.

[Z94] R. J. ZimmeR, Discrete groups and non-Riemannian homogeneous spaces, J. Amer. Math. Soc. 7 (1994), 159-168.

Research Institute for Mathematical Sciences

Kyoto University, Sakyo-ku Kyoto, 606-8502, Japan

E-mail address: toshi@kurims.kyoto-u.ac.jp

yoshino@kurims.kyoto-u.ac.jp 